\title{
NUMERICAL INVESTIGATION OF HEAT TRANSFER AND SOLIDIFICATION IN A CAVITY FILLED WITH MOLTEN METAL ALLOYS IN THE PRESENCE OF THERMODIFFUSION
}

\author{
by \\ Elham Jafar-Salehi \\ Bachelor of Material Engineering, University of Tehran, Tehran, Iran, 1999 \\ Master of Mechanical Engineering, Ryerson University, Toronto, Canada, 2010
}

A dissertation presented to

Ryerson University

in partial fulfillment of the requirements for the degree of

Doctor of Philosophy

in the Program of

Mechanical \& Industrial Engineering

Toronto, Ontario, Canada, 2015

(c) Elham Jafar-Salehi 2015 


\section{Author's Declaration}

I hereby declare that I am the sole author of this dissertation. This is a true copy of the dissertation, including any required final revisions, as accepted by my examiners.

I authorize Ryerson University to lend this dissertation to other institutions or individuals for the purpose of scholarly research.

I further authorize Ryerson University to reproduce this dissertation by photocopying or by other means, in total or in part, at the request of other institutions or individuals for the purpose of scholarly research.

I understand that my dissertation may be made electronically available to the public. 


\begin{abstract}
Numerical Investigation of Heat Transfer and Solidification in a Cavity Filled with Molten Metal Alloys in the Presence of Thermodiffusion
\end{abstract}

\author{
Doctor of Philosophy
}

2015

\title{
Elham Jafar-Salehi
}

\section{Mechanical \& Industrial Engineering \\ Ryerson University}

The objective of this research was to study the solidification process of binary molten metals. This study was conducted in three phases. One was to estimate the thermodiffusion factor, two was to simulate the effect of natural convection and radiation on the velocity, temperature, and concentration distributions, and the third was to investigate the solidification process of binary molten metals using the proposed thermodiffusion factors.

The proposed expression for the estimation of thermodiffusion factor was based on the physical properties of the mixture constituents. The estimated thermodiffusion factor was used to study thermosolutal convection in a quartz enclosure filled with molten Sn-Bi alloy. Two simulations were carried out: top heating and bottom heating. The sidewalls in both cases were exposed to convection and radiation. Numerical results show that in the case of top heating, the distribution of temperature and concentration are linear, but species segregation occurs due to the thermodiffusion effect. In the bottom heating case, boundary-driven convective flow develops with a large Rayleigh number (Ra) where an increase in the Ra number negates thermodiffusion 
due to the development of strong mixing. The results of these simulations showed that the effect of convection and radiation are negligible.

In phase three, finite element method (FE) was employed to investigate the effect of thermodiffusion during vertical solidification of binary molten metal alloys with bottom cooling. The systems considered here are: tin-bismuth (Sn-Bi), tin-cadmium (Sn-Cd), tin-zinc (Sn-Zn), tin-lead ( $\mathrm{Sn}-\mathrm{Pb})$, tin-gallium (Sn-Ga), and bismuth-lead (Bi-Pb) binary molten metals. The geometry under study was a cylindrical cavity. The FE model was constructed using a 2D axisymmetric element to represent a 3D cylindrical model. Two cases were studied: one without and one with the effect of thermodiffusion. The simulation including thermodiffusion showed slight variation from the simulation without thermodiffusion, in that thermodiffusion causes a slightly faster solidification and a more uniform concentration distribution if the thermodiffusion coefficient is greater than zero $\left(D_{T}>0\right)$. The main object of this research is development of a more accurate thermodiffusion factor, and applying it in a numerical simulation to study its effects on radiation, natural convection, and solidification processes. 


\section{Acknowledgements}

I would like to give my special thanks and gratitude to Professor Dr. Ziad Saghir for his guidance and support during my PhD program; I am grateful to him for his support and countless advice and allowing me to work on this extremely interesting and yet challenging research project. I would like to further extend my special thank to Dr. Morteza Eslamian for his valuable inputs. I acknowledge the financial support of the Natural Sciences and Engineering Research Council of Canada (NSERC) and the School of Graduate Studies in Ryerson University.

Most importantly, I would like to thank my beloved husband, Hamed Tabatabaei and my two children Kevin and Erin for their unconditional love and support. This work could not have been attained without their support and words of encouragement. At times that I had no strength to go on, they were there to lend moral support, for that I am forever indebted to them.

I wish to dedicate this work to my hero, my protector and my true teacher my father, Bahadorgholi Jafar-Salehi as well as my sister Afsaneh Jafar-Salehi. Even though they are with God and looking over me, their memory gave me strength to fulfill my dream. Here you are father, I am sure you are proud of me as I always am proud of being your daughter. 


\section{Table of Contents}

Author's Declaration .............................................................................................................................. ii

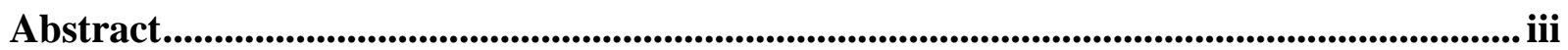

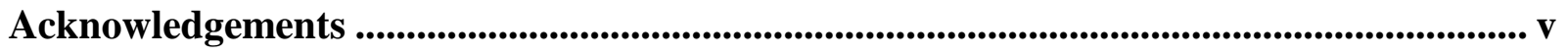

Table of Content.................................................................................................................................... vi

List of Tables ............................................................................................................................ ix

List of Figures..................................................................................................................................... $\mathrm{x}$

List of Appendices.................................................................................................................................. xiv

Nomenclature ....................................................................................................................................... XV

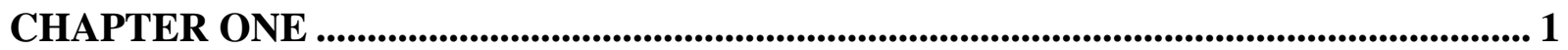

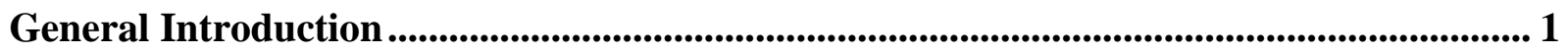

1.1 Introduction .......................................................................................................... 1

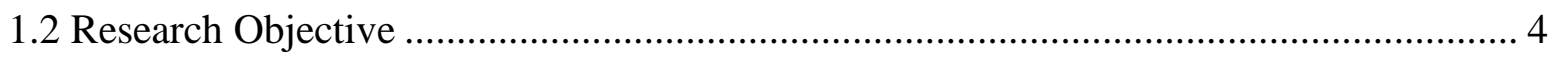

1.3 Motivation and Need for this Research ……………..................................................... 5

1.4 Dissertation Organization ................................................................................... 8

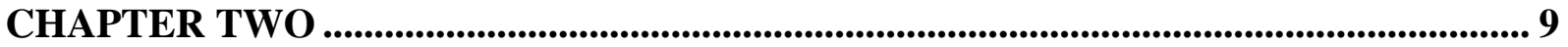

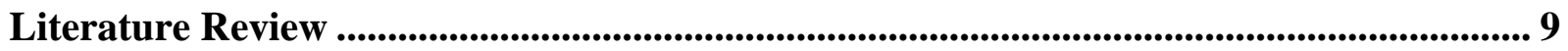

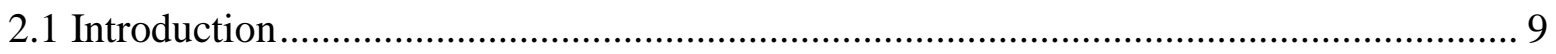

2.2 Theoretical Model for Thermodiffusion Factor .............................................................. 10

2.3 Natural Convection and Surface Radiation................................................................. 22

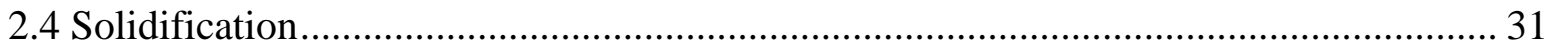

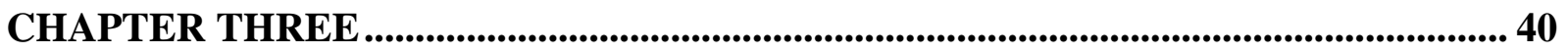




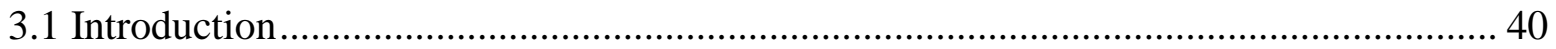

3.2 Model Development and Derivation............................................................................. 40

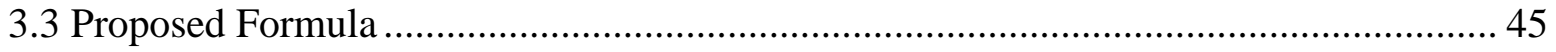

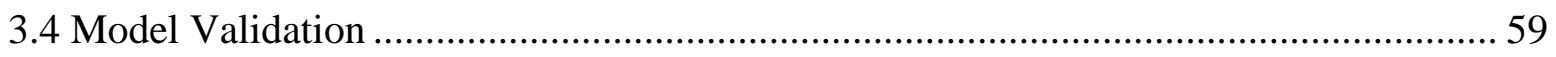

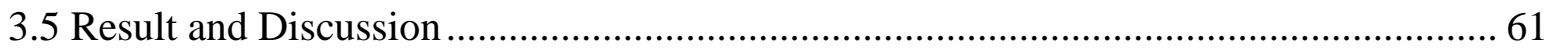

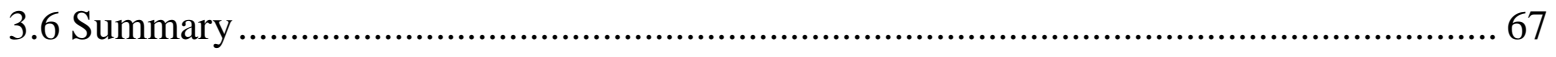

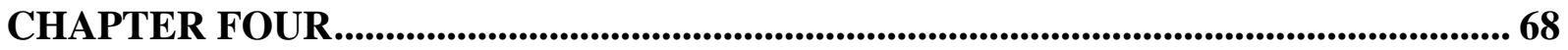

The Role of Convection and Radiation Coupled with Thermodiffusion Effect in Binary

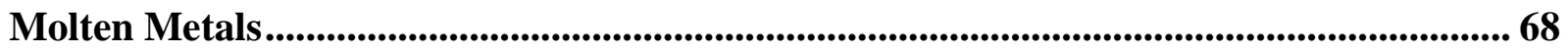

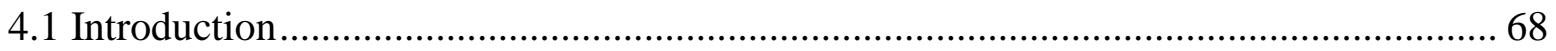

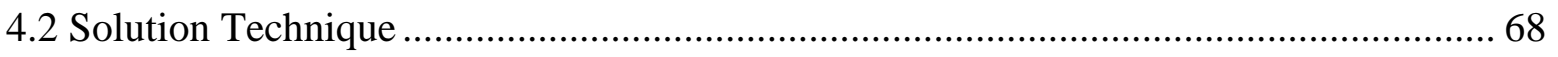

4.3 Governing Equations and Boundary Condition .......................................................... 70

4.4 Numerical Modeling of Natural Convection ……………............................................. 73

4.5 Model Geometry and Boundary Conditions ................................................................. 74

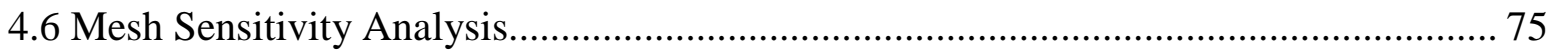

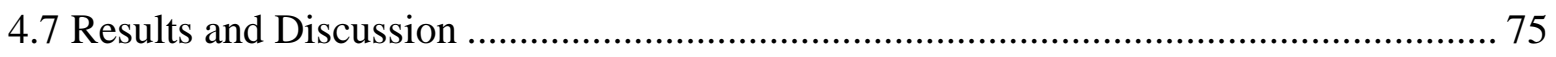

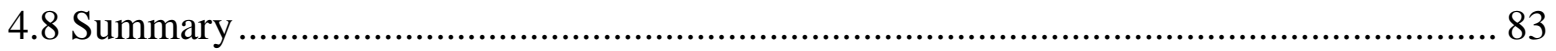

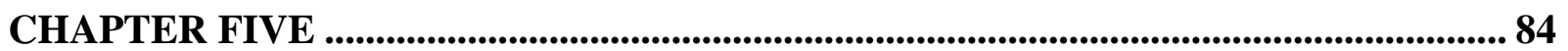

Influence of Thermodiffusion Effect on Solidification of Binary Molten Alloy Utilizing Finite Element Method .................................................................................................................. 84

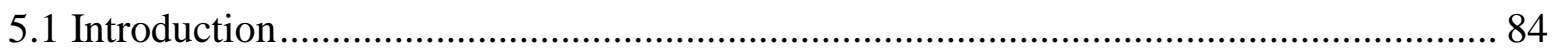

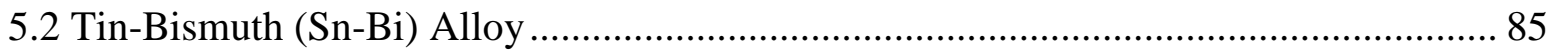




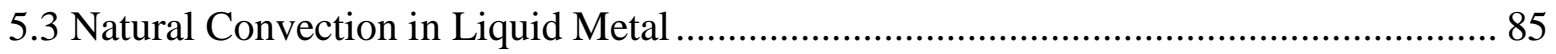

5.4 Thermodiffusion Effect on Solidification ....................................................................... 87

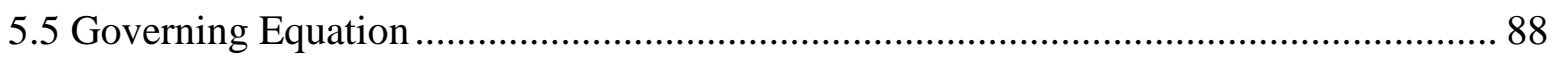

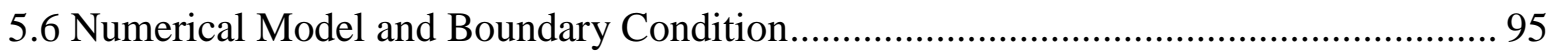

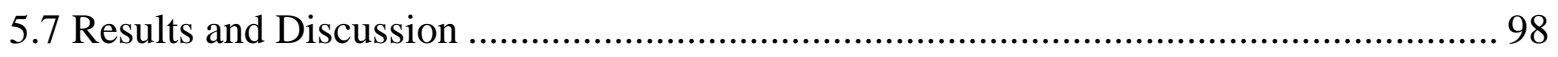

5.8 Influence of Material Properties on Solidification Progress........................................ 108

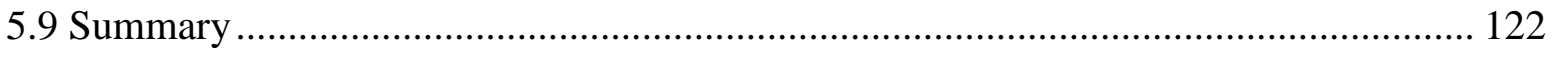

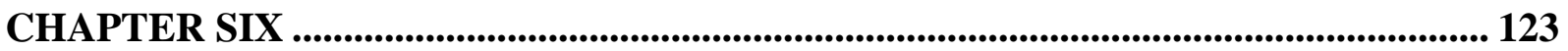

Summary, Conclusion and Future Work ................................................................................ 123

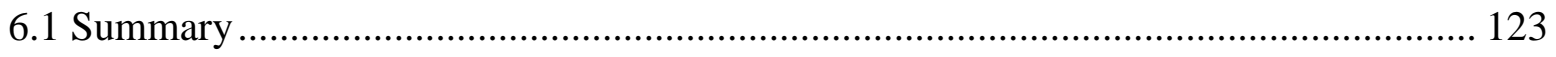

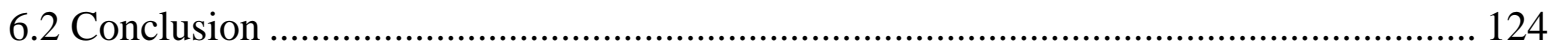

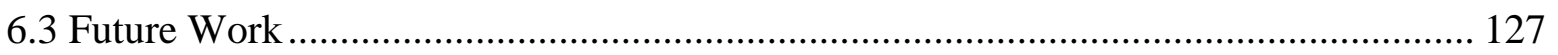

APPENDIX A ........................................................................................................................... 128

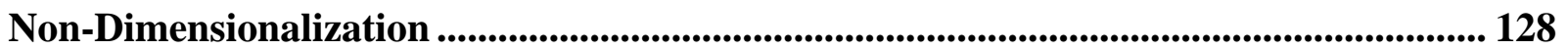

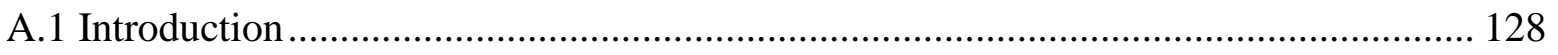

A.2 Dimensionless Form of Governing Equations ........................................................... 129

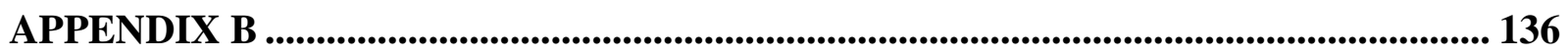

Heaviside Function............................................................................................................................... 136

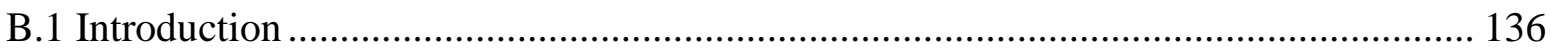

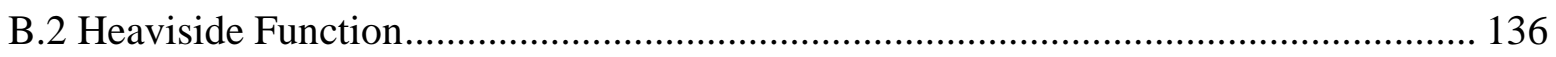

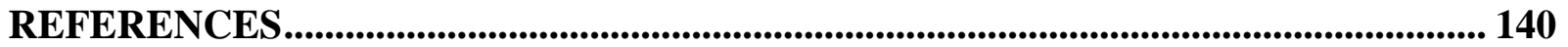




\section{List of Tables}

Table 1 - 1: Flux-force coupling between heat and mass................................................... 6

Table 3 - 1: Fermi energy and thermoelectric power parameter of selected molten metals........ 45

Table 3 - 2: Activation energy of viscouse flow of selected molten metals at various tempretures.

Table 3 - 3: Estimated partial molar volumeVi, enthalpies $H i$, and activity parameter $\partial \mu / \partial x 1$ of mixture component at given mole fraction of the first component $(x i)$ and temperature for selected mixtures.

Table 3 - 4: Comparison of calculated thermodiffusion factor against the experimental

data.

Table 3 - 5: Experimental and calculated using Maier model (from Eq. 3.20) and modified model with Electron-Ion forces against the experimental data.

Table 4 - 1: The physical properties of Sn-Bi mixture at $295^{\circ} \mathrm{C}$ [17] and the references therin... 73

Table 5 - 1: The Physical Properties of Sn-Bi Molten alloy at $235^{\circ} \mathrm{C}$ [25] 97

Table 5 - 2: The Physical properties of binary molten alloys at $235^{\circ} \mathrm{C}$ [165]...... 109

Table 5 - 3: Variation of concentration for five binary molten metal for with and without thermodiffusion effect. 


\section{List of Figures}

Figure 3- 1: Effect of concentration on thermodiffusion factor: Comparison of the prediction of different models. Note that the experimental data is only available for an equimolar mixture. (a) Sn-Bi at 568 K, (b) Sn-Cd at 568 K, (c) Sn-Zn at 568 K, (d) Sn-Pb at 568 K, (e) Sn-Pb at $723 \mathrm{~K}$, (f) Bi-Pb at $568 \mathrm{~K}$ and (g) Sn-Ga at $568 \mathrm{~K}$. 66

Figure 3- 2: Thermodiffusion coefficient of binary molten metals at various temperatures and $x 1=0.5$ by using Stokes-Einstein equation for each component and the proposed model for: Sn-Bi, Sn-Pb, Sn-Ga, Bi-Pb, and Na-k.

Figure 4 - 1: Computational cell geometry and boundary conditions.....................74

Figure 4 - 2: Temperature and concentration distribution with top heating condition; (a) Lines of constant temperature; (b) Temperature distribution along the cavity height; (c) Lines of constant concentration; (d) Concentration variation along the cavity height.

Figure 4 - 3: Comparison of (a) temperature distribution, and (b) Concentration distribution by applying surface radiation and external natural convection on vertical walls to the ambient in Sn-Bi filled square cavity with thermodiffusion effect at $\mathrm{Ra}=1000$.

Figure 4 - 4: Temperature and concentration distribution in the cell in bottom heating; (a) Constant temperature lines in the cavity; (b) Constant concentration lines in the cavity..... 82

Figure 4 - 5: Variation of concentration at different Rayleigh numbers in bottom heating. ....... 83

Figure 5 - 1: Schematic of the two-phase nature of a solidifying mushy region [106]..........89

Figure 5 - 2: Schematic diagram of the model for molten alloy......................................... 96

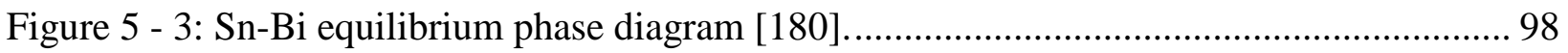


Figure 5 - 4: Simulated result for temperature distribution at 25\% solidification of Pb-0.35Sn; (a)

Our reproduced simulation, (b) Extracted from published research... 99

Figure 5 - 5: comparison of the simulated result for composition distribution at 25\% solidification of $\mathrm{Pb}-0.35 \mathrm{Sn}$. 99

Figure 5 - 6: Simulated result for streamlines at 25\% solidification of Sn-Bi; (a) without thermodiffusion simulation, (b) with thermodiffusion. 104

Figure 5 - 7: Simulated result for temperature distribution at 25\% solidification of Sn-Bi; (a) without thermodiffusion simulation, (b) with thermodiffusion 105

Figure 5 - 8: Simulated result for liquid fraction at 25\% solidification of Sn-Bi; (a) without thermodiffusion simulation, (b) with thermodiffusion 105

Figure 5 - 9: Simulated result for composition distribution at 25\% solidification of Sn-Bi; (a) without thermodiffusion simulation, (b) with thermodiffusion 106

Figure 5 - 10: The time dependence temperature at the point $\mathrm{z}=50 \mathrm{~mm}$ above the bottom of the cavity for two cases; without /with thermodiffusion. 107

Figure 5 - 11: The line dependence of volume fraction of liquid phase at point $\mathrm{z}=50 \mathrm{~mm}$ above the bottom of the cavity for two cases; without /with thermodiffusion. 107

Figure 5 - 12: Simulated result for streamlines at 25\% solidification of Sn-Zn; (a) without thermodiffusion simulation, (b) with thermodiffusion.

Figure 5 - 13: Simulated result for streamlines at 25\% solidification of Sn-Pb; (a) without thermodiffusion simulation, (b) with thermodiffusion.

Figure 5 - 14: Simulated result for streamlines at 25\% solidification of Sn-Cd; (a) without thermodiffusion simulation, (b) with thermodiffusion. 
Figure 5 - 15: Simulated result for streamlines at 25\% solidification of Bi-Pb; (a) without thermodiffusion simulation, (b) with thermodiffusion.

Figure 5 - 16: Simulated result for streamlines at 25\% solidification of Sn-Ga; (a) without hermodiffusion simulation, (b) with thermodiffusion.

Figure 5 - 17: Simulated result for temperature distribution at 25\% solidification of Sn-Zn; (a) without thermodiffusion simulation, (b) with thermodiffusion. 114

Figure 5 - 18: Simulated result for temperature distribution at 25\% solidification of Sn-Pb; (a) without thermodiffusion simulation, (b) with thermodiffusion.

Figure 5 - 19: Simulated result for temperature distribution at 25\% solidification of Sn-Cd; (a) without thermodiffusion simulation, (b) with thermodiffusion.

Figure 5 - 20: Simulated result for temperature distribution at 25\% solidification of Bi-Pb; (a) without thermodiffusion simulation, (b) with thermodiffusion.

Figure 5 - 21: Simulated result for temperature distribution at 25\% solidification of Sn-Ga; (a) without thermodiffusion simulation, (b) with thermodiffusion. 116

Figure 5 - 22. Progress of solidification for binary molten metals; Sn-Zn, Sn-Pb, Sn-Cd, Bi-Pb, and Sn-Ga.

Figure 5 - 23: Simulated result for composition distribution at 25\% solidification of Sn-Zn; (a) without thermodiffusion simulation, (b) with thermodiffusion 118

Figure 5 - 24: Simulated result for composition distribution at 25\% solidification of Sn-Pb; (a) without thermodiffusion simulation, (b) with thermodiffusion 118

Figure 5 - 25: Simulated result for composition distribution at 25\% solidification of Sn-Cd; (a) without thermodiffusion simulation, (b) with thermodiffusion 
Figure 5 - 26: Simulated result for composition distribution at 25\% solidification of Bi-Pb; (a) without thermodiffusion simulation, (b) with thermodiffusion 119

Figure 5 - 27: Simulated result for composition distribution at 25\% solidification of Sn-Ga; (a) without thermodiffusion simulation, (b) with thermodiffusion ..................................... 120 


\section{List of Appendices}

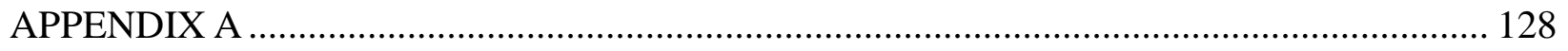

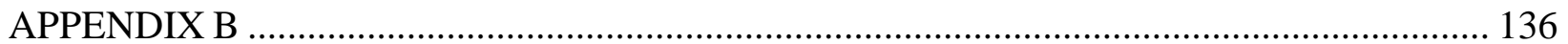




\section{Nomenclature}

$c_{j} \quad$ Mass fraction of mixture component $j(-)$

C Non-dimensional concentration of a mixture component (-)

D Molecular diffusion coefficient $\left(\mathrm{cm}^{2} . \mathrm{s}^{-1}\right)$

$D_{T} \quad$ Thermodiffusion coefficient $\left(\mathrm{cm}^{2} \cdot \mathrm{s}^{-1} \cdot \mathrm{K}^{-1}\right)$

$E_{i}^{v i s} \quad$ Activation energy of viscous flow of the component $i\left(\mathrm{j} \cdot \mathrm{mol}^{-1}\right)$

$g \quad$ Gravitational acceleration $\left(\mathrm{cm} . \mathrm{s}^{-2}\right)$

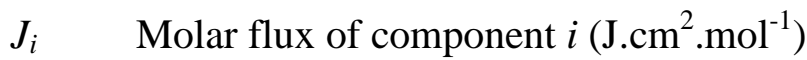

$L_{i k} \quad$ Phenomenological coefficient

p $\quad$ Pressure $(\mathrm{Pa})$

$P \quad$ Non-dimensional pressure

$\mathrm{K}$ Thermal conductivity $\left(\mathrm{J} \cdot \mathrm{s}^{-1} \cdot \mathrm{cm}^{-1} \cdot \mathrm{K}^{-1}\right)$

$\mathrm{C}_{\mathrm{p}} \quad$ Specific heat capacity $\left(\mathrm{J} \cdot \mathrm{g}^{-1} \cdot \mathrm{K}^{-1}\right)$

$Q_{k}^{*} \quad$ Heat of transport of component k $\left(\mathrm{J} \cdot \mathrm{mol}^{-1} \cdot \mathrm{K}^{-1}\right)$

$S_{T} \quad$ Soret Coefficient $=\frac{D_{T}}{D}\left(K^{-1}\right)$

T Temperature (K)

$u \quad$ Velocity component in the $\mathrm{x}$ direction $\left(\mathrm{cm} . \mathrm{s}^{-1}\right)$

$U \quad$ Non-dimensional velocity component in the X direction (-)

$u_{o} \quad$ Characteristic velocity $=\sqrt{g \cdot \beta_{T} \cdot \Delta T \cdot L}\left(\mathrm{~cm} \cdot \mathrm{s}^{-1}\right)$

$v \quad$ Velocity component in the y direction $\left(\mathrm{cm} . \mathrm{s}^{-1}\right)$

$V \quad$ Non-dimensional velocity component in the Y direction (-) 
$x_{i} \quad$ Mole fraction of a mixture component $i$

$S_{\text {Is }}(\mathrm{T})$ Partial specific entropy difference (J.K $\left.{ }^{-1}\right)$

$S_{i} \quad$ Entropy of component $i\left(\mathrm{~J}^{\mathrm{K}} \mathrm{K}^{-1}\right)$

|e $\quad$ Absolute value of an electron

$\mathrm{Z}_{i} \quad$ Valence of ion $i$

$\mathrm{V}_{i} \quad$ Molar volume of component $i\left(\mathrm{~cm}^{3} \cdot \mathrm{mol}^{-1}\right)$

$\mathrm{m}_{i} \quad$ Molar mass of component $i\left(\mathrm{~g} \cdot \mathrm{mol}^{-1}\right)$

$R \quad$ Gas constant. $\left(\mathrm{J} \cdot \mathrm{mol}^{-1} \cdot \mathrm{K}^{-1}\right)$

$N \quad$ Avogadro number

$\mathrm{K}_{B} \quad$ Boltzman constant (J.K $\left.\mathrm{K}^{-1}\right)$

$\mathrm{E}_{f} \quad$ Fermi energy $(\mathrm{eV})$

$D_{m} \quad$ Molecular diffusion coefficient $\left(\mathrm{cm}^{2} . \mathrm{s}^{-1}\right)$

$D_{T} \quad$ Thermodiffusion coefficient $\left(\mathrm{cm}^{2} \cdot \mathrm{s}^{-1} \cdot \mathrm{K}^{-1}\right)$

$g \quad$ Gravitational acceleration( $\left(\mathrm{cm}^{\mathrm{s}} \mathrm{s}^{-2}\right)$

$\vec{J} \quad$ Thermodiffusion flux $\left(\mathrm{J} . \mathrm{cm}^{2} \cdot \mathrm{mol}^{-1}\right)$

$p \quad$ Pressure (Pa)

$\mathrm{K}$ Thermal conductivity $\left(\mathrm{J} . \mathrm{s}^{-1} \cdot \mathrm{cm}^{-1} \cdot \mathrm{K}^{-1}\right)$

$\mathrm{C}_{\mathrm{p}} \quad$ Specific heat capacity $\left(\mathrm{J} \mathrm{g}^{-1} \cdot \mathrm{K}^{-1}\right)$

$S_{T} \quad$ Soret Coefficient $=\frac{D_{T}}{D}\left(K^{-1}\right)$

$T_{m} \quad$ Melting Temperature (K)

$\frac{\partial D}{\partial T} \quad$ Temperature Coefficient $\left(\mathrm{cm}^{2} \cdot(\mathrm{s} . K)^{-1}\right)$

$r \quad$ Radial Coordinate

z Vertical Coordinate 


$\begin{array}{ll}C & \text { Solute Concentration (wt\%) } \\ \mathrm{T}_{\mathrm{e}} & \text { Eutectic Temperature (K) } \\ \mathrm{L} & \text { Latent Heat }\left(\mathrm{J} .(\mathrm{kg})^{-1}\right) \\ \mathrm{D}_{\mathrm{a}} & \text { Darcy Number } \\ \mathrm{k} & \text { Permeability }\left(\mathrm{m}^{2}\right) \\ \mathrm{f}_{\mathrm{l}} & \text { Liquid Fraction } \\ \mathrm{f}_{\mathrm{s}} & \text { Solid Fraction } \\ \mathrm{t} & \text { Time (s) } \\ R_{r} & \text { Darcian Resistance Term in } r \text { Direction } \\ R_{z} & \text { Darcian Resistance Term in } z \text { Direction } \\ B_{z} & \left.\text { Body Force (N.m }{ }^{-3}\right) \\ \varepsilon & \text { Porosity } \\ B & \text { Volume Fraction } \\ C_{\text {ref }} & \text { Reference Solute Concentration } \\ \mathrm{T}_{\mathrm{ref}} & \text { Reference Temperature (K) }\end{array}$

\section{Non-dimensional numbers}

Pr Prandtl number $=\frac{v}{\alpha}$

Re $\quad$ Reynold number $=\frac{\rho_{o} \cdot u_{o .} L}{\mu}$

Sc $\quad$ Schmidt number $=\frac{\mu}{\rho . D}$

Ra $\quad$ Rayleigh number $=\frac{g \cdot \beta}{v \cdot \alpha} \cdot \Delta T \cdot L^{3}$ 


\section{Greek Symbol}

$\alpha_{T} \quad$ Thermodiffusion factor $=T \frac{D_{T}}{D}$

$\alpha \quad$ Thermal diffusivity $\left(\mathrm{cm}^{2} . \mathrm{s}^{-1}\right)$

$\beta c \quad$ Solutal Expansion (-)

$\beta_{T} \quad$ Thermal volume expansion $\left(\mathrm{K}^{-1}\right)$

$\theta \quad$ Non-dimensional temperature $=\frac{T-T_{C}}{\Delta T}$

$\mu_{k} \quad$ Chemical potential of component $k\left(\mathrm{~J} \mathrm{~mol}^{-1}\right)$

$\eta \quad$ Dynamic viscosity $\left(\right.$ g. $\left.\mathrm{cm}^{-1} \cdot \mathrm{s}^{-1}\right)$

$v \quad$ Kinematic viscosity $\left(\mathrm{cm}^{2} \cdot \mathrm{s}^{-1}\right)$

$\rho_{o} \quad$ Density of fluid at reference temperature $T_{\mathrm{o}}\left(\mathrm{g} \cdot \mathrm{cm}^{-3}\right)$

$\Delta c_{j} \quad$ Concentration (mass fraction) difference of component $j(-)$

$\Delta T \quad$ Temperature difference $=T_{H}-T_{C}(\mathrm{~K})$

$\nabla \quad$ Spatial gradient of a variable

\section{Subscript}

C Cold side of mixture

$\mathrm{H} \quad$ Hot side of mixture

o $\quad$ Reference 


\section{CHAPTER ONE}

\section{General Introduction}

\subsection{Introduction}

Natural convection in a closed cavity is a rather complex and interesting subject in heat transfer. It has been studied mainly in two-dimensional rectangular enclosures where a temperature gradient has been applied across the enclosure from two opposing walls, either vertically or horizontally. This heating has traditionally taken the form of heating from below (Rayleigh-Benard convection) or heating applied from one vertical wall and cooling on the opposing wall. Depending on the configuration, the temperature gradient can cause a boundarydriven flow.

The complexity of fluid flow and heat transfer within the cavity increases when a binary system is considered, and when radiation is present it can be even more complex, since the assumption of adiabatic boundary conditions no longer holds. The temperature gradient acting on a binary system, such as a two-component molten metal alloy, causes separation of the constituents in a process called thermodiffusion. This makes the modeling and prediction of flow in a binary system much more complex that for a system with a single component.

Natural convection is very common in practice. It occurs when a fluid moves across a surface with a temperature variation. These temperatures difference cause density variations in the liquid, and results in fluid flow over the surface owing to the buoyancy force arising from the density differences inside the liquid. Natural convection can be either an internal flow, like a flow in a container such as a pipe, or an external flow, such as fluid flowing up a heated wall. 
Since the 1980s, natural convection within enclosed cavities has been the focus of many studies due to its diverse applications in single crystal growth systems, semi-conductor industries, solar collectors, heating and cooling of buildings, and thermal pollution dispersion in lakes or estuaries [1]. Another equally important factor that can interact with natural convection is thermal radiation. The influence of radiation can be neglected in studies that use geometries with polished surfaces, which cause a very weak emissivity. In general, radiation has a greater influence on natural convection than forced convection due to the inherent coupling that exists between the temperature and flow fields in natural convection. Coupled natural convection and radiation occur in furnaces, natural water bodies, solar energy utilization, crystal growth, etc. There are numerous experimental and numerical studies on natural convection of an enclosed cavity that are available in literature. However, very few of these studies consider the influence that surface radiation has on natural convection, particularly in cases where the fluid contained in the enclosure is radiatively nonparticipating.

Understanding the effects of natural convection on the flow motion and the resulting interface shape between solids and liquids is of critical importance in designing and optimizing solidification systems for material processing. Nowadays, about $80 \%$ the commercially available materials are grown from a molten state. Advanced technical fields such as the aerospace industry and the optical and electronic industries need high quality crystals with low levels of defects and high levels of solute uniformity. Previous studies have indicated that the combined effects from imposed thermal gradients and gravity result in improper convective flow patterns in the melt. This has been identified as a major cause of structural defects and nonhomogeneity in crystals [1]. During solidification, the microscopic flow and heat and / or mass transfer within the growing dendritic structures near the solidified front are strongly affected by 
the macroscale bulk motion in the liquid pool. This gives rise to unsteady temperature and concentration distributions in the molten zone, which in turn causes striation and nonhomogeneity in the solid and finally results in crystals unsuitable for high quality requirements [2]. It is important to note that solidification processes play a vital role in the metallurgy of the solidified metal, and they often dictate the physical and mechanical properties of the resulting solid materials. Major procedures that use this process are obtaining ingots of ferrous and nonferrous metals, growing of semiconductor crystals, synthesizing optical crystals, obtaining ultra-pure materials, etc. Even though extensive research has been performed on solidification process, many attributes of the physics of solidification still remain unclear, such as various types of micro- and macrostructures in solids. For this reason, the current thesis considers a model complicated by the Soret effect and temperature dependent diffusion.

The Ludwig-Soret effect, also known as thermodiffusion, thermal diffusion, thermomigration, or the Soret effect (more generally, the name thermal diffusion is used to describe this effect in a gas mixture, whereas the Soret effect or the Ludwig effect is used in liquids), is a classic example of coupled heat and mass transport in which the motion of the particles in a fluid mixture is driven by a heat flux coming from a thermal gradient. Most often, the heaviest particles move from hot to cold, but the reverse is also seen under some conditions. In the past 150 years, the Soret effect has been studied in some particularly active time periods motivated by economic interests, e.g., the separation of isotopes in the 30s and petroleum engineering in the 90s, to name a few [3]. Many researchers have developed different techniques to measure this effect and theories to explain it. However, because of the complexity of this coupled phenomenon, only recently, there has been an agreement on the values of the thermal diffusion coefficients measured by different techniques. Theoretically, there exists a rigorous 
approach based on the kinetic gas theory, which explains the thermal diffusion effect for binary and multi-component ideal gas mixtures. For liquids, the theories developed are not precise enough to fully model the phenomenon, and there is still a lack of understanding of the basis of the effect for liquid mixtures. The situation becomes even more complex when considering solidification in these systems.

\subsection{Research Objective}

The present research addressed three objectives, which are met through the research described in chapters 3,4 , and 5 , respectively: The first objective was to determine more accurate thermodiffusion factors; the second objective was to study the effects of external natural convection and surface radiation in the presence of thermodiffusion on the temperature, velocity, and concentration distributions; and the third objective was to the simulate the effect of thermodiffusion on solidification of binary molten metals using the finite element technique.

In chapter 3, an expression was derived to estimate a thermodiffusion factor for binary molten metal alloys based on a linear non-equilibrium thermodynamics formulation. The expression contains two terms: The first term explains the interaction due to a temperature gradient between the two species, and the second term describes an electronic contribution, which is equivalent to the mass diffusion caused by an internal electric field induced as a result of the thermal gradient. In chapter 4, the external natural convection and surface radiation to the ambient in rectangular quartz cavity filled with binary molten metal alloys were modeled to include the effect of the thermodiffusion. The estimated thermodiffusion factors for molten metal alloys were applied to simulate the external natural convection and surface radiation in a 2D rectangular quartz cavity filled with a tin-bismuth (Sn-Bi) alloy. The solidification of Sn-35 
wt.\% Bi alloy in a cavity was studied by solving the heat transfer, fluid flow, and species equations, and by using the finite element method (FE) in the presence of thermodiffusion. Also, this simulation was further extended in chapter 5 to study the solidification processes for five more binary molten metals whose thermodiffusion factors were determined as part of this work. These binary systems were tin-cadmium (Sn-Cd), tin-zinc ( $\mathrm{Sn}-\mathrm{Zn})$, tin-lead (Sn-Pb), tin-gallium ( $\mathrm{Sn}-\mathrm{Ga}$ ) and bismuth-lead (Bi-Pb). To the best of our knowledge, there is no other work that considers thermodiffusion in the convective flow of molten metal alloys.

\subsection{Motivation of this Research}

The effect of thermodiffusion during the solidification of binary molten metal mixtures has not been recently studied. However, the thermodiffusion effect exists during this process and influences the microstructure of the end product. Furthermore, it is important to consider the external natural convection and surface radiation in order to determine their influence on the solidification process. Therefore, the current work is to identify and address the limitations of the existing models and propose an accurate model for the estimation of thermodiffusion factors in systems consisting of binary molten metals. Ultimately, the goal of this research is the study of convection and radiation in a vertical solidification process of binary molten metals in the presence of thermodiffusion.

\subsubsection{Industrial Interest of the Soret Effect}

In order to optimize production costs when extracting from a fluid field, it is important to know precisely the distribution of the different species in the field. This distribution is usually generated over a long formation period with the separation mainly influenced by gravity and the 
distribution of pressure in the reservoir. Various methods have been implemented in order to obtain reliable thermodynamic models of the constituents in a reservoir. Since it is not possible to ignore the important vertical extension of a given field, it is highly probable that this distribution is influenced by both thermal diffusion and convection.

The temperature gradient causes the migration of species through the Soret effect. This is the creation of a concentration gradient of the chemical composition by the presence of a thermal gradient, i.e., the existence of a thermal gradient is causing migration of species. This effect, discovered by C. Ludwig in 1856 [4] and better described by C. Soret in 1880 [5], is a particular phenomenon since it is associated with several thermodynamic phenomena. It is a flux-force coupling phenomenon, that is, a flux created by a force of different nature, in this case, a concentration gradient induced by the presence of a thermal gradient. Table 1.1 summarizes the flux-force coupling effects for heat and mass transfer.

Table 1 - 1: Flux-force coupling between heat and mass [5].

\begin{tabular}{lll}
\hline Flux/Force & \multicolumn{1}{c}{$\nabla T$} & \multicolumn{1}{c}{$\nabla C$} \\
\hline Heat & Fourier’s Law of Conduction & Dufour effect \\
\hline Mass & Soret effect & Fick’s law of diffusion \\
\hline
\end{tabular}

The study of the relations between fluxes and forces of this type is called thermodynamics of linear irreversible processes [6]. The main characteristic quantity for thermal diffusion is a coefficient called the Soret coefficient $\left(S_{T}\right)$. Many studies have been performed to determine this quantity using different approaches: Experimental approaches, for example, Soret coefficients in crude oil in microgravity $[7,8]$ and using a thermo-gravitational column; or theoretical approaches, such as using molecular dynamics simulations $[9,10]$ or multicomponent numerical models [11]. Generally, the theoretical values differ from the values found 
experimentally. The differences are mainly due to the fact that the measurements are technically simpler in a medium without the mushy zone. Failure in the thermo-gravitational model based on free fluid equations is a good example of the need to determine a new model for describing the phenomena of molecular diffusion and thermal diffusion during the process of solidification.

\subsubsection{Industrial Applications of Solidification}

Solidification is an extremely important manufacturing process whose applications include casting, welding, laser processing, and crystal growth. Solidification is controlled by various phenomena that closely interact with each other, such as transport of energy and solute, convection, nucleation, growth, etc. The properties of solidified materials, such as segregation, porosity, structure, and strength are controlled by one or more of these factors [12]. In most cases, the transformation of liquid to solid occurs in the presence of natural convection. The common causes of natural convection are thermal buoyancy, solutal buoyancy, shrinkage, etc. The flow can also be activated by means of external forces. It is understood that during the solidification process, the flow in the mushy region significantly influences the properties of the solid products. Some of these special effects are listed below:

- Morphology of solid-liquid interface: Flow significantly affects the transition from planar to cellular and cellular to dendritic interface.

- Microstructure: Flow affects the microstructure by changing the cooling rate and solute concentration.

- Macrostructure: Flow also can significantly affect the morphology and morphological transition (columnar-to-equiaxed transition, for example).

- Segregation: Macrosegregation is determined by convection in the mushy zone and superheated regions. It is affected by flow. 
- Microsegregation: It is caused primarily by diffusion. However, it is affected by the morphology and the solute concentration.

- Freckles: This defect is related to the plumes of solute-rich liquid originating from channels in the mushy region.

The study of thermodiffusion in molten metal (also known as thermo-transport and thermomigration) was driven by its potential applications in the isotopic enrichment of materials [12], design of nuclear reactors that experience high temperatures [13], and other nuclear-related activities [14,15].

\subsection{Dissertation Organization}

The background and main objective of this research was introduced in this chapter. The remainder of this research work is organized as follows:

- Chapter 2 presents a comprehensive literature review of the thermal diffusion effect, natural convection, and surface radiation as well as of the solidification process in binary systems of molten metal.

- Chapter 3 presents the derivation of the proposed thermodiffusion factors along with other widely used theoretical models.

- Chapter 4 explains the effects of external natural convection and surface radiation on a binary molten metal system in a quartz cavity, taking into account thermodiffusion.

- Chapter 5 presents the simulation of the solidification process of binary molten metal alloys using finite element software.

- Chapter 6 outlines the summary, conclusions, and future work of this research. 


\section{CHAPTER TWO}

\section{Literature Review}

\subsection{Introduction}

For the past three decades, scientists in fields related to convection have focused their attention on the natural convection inside cavities. This is due to the importance of single crystal growth in various applications such as semi-conductor industries, solar collectors, and heating and cooling of buildings [16]. Natural convection, surface radiation, and solidification in rectangular cavities have been studied numerically and experimentally for several decades, but studies have not included thermodiffusion. In other words, various simulations have been carried out to solve different cases of convection without the inclusion of the Soret effect and temperature dependent diffusion. However, this is a simplified view of the problem, since temperature gradients imposed on a binary mixture causes separation of the species from one another in a phenomenon called thermodiffusion. Thermodiffusion, or the Soret effect, is one of the simultaneous heat and mass transfer phenomena that occurs in a solid, liquid, or gas mixture, as a result of an external temperature gradient imposed across the mixture. Thermodiffusion may be present and utilized in several non-isothermal phenomena and processes.

This literature review will only consider the work closely related to the present research of laminar natural convection, surface radiation, and solidification due to differentially heated end walls. The second section in this chapter will describe the pertinent literature and the methods through which the thermodiffusion factor for binary component mixtures has been derived. The third section will review natural convection and surface radiation, and the fourth section will discuss the studies on solidification in the presence of a constant temperature 
gradient.

\subsection{Theoretical Model for Thermodiffusion Factor}

A few researchers have considered the problem of thermodiffusion in molten metal alloys from a theoretical perspective and consequently have presented various models to describe it. A great deal of debate exists surrounding this topic due to inaccuracies in measurements and discrepancies in the theories. It is, however, agreed that the reason for mass diffusion in general is the spatial concentration of species (also known as chemical potential difference) within the mixture. In a homogeneous solution, temperature or pressure gradients contribute to the mass diffusion in the process of thermodiffusion. Generally, the thermodiffusion factor $\alpha$, the Soret coefficient $S_{T}$, and the thermodiffusion coefficient $D_{T}$ are associated with each other and with the molar flux of component $1\left(J_{1}\right)$ through the modified Fick's law [17]:

$J_{1}=-c D\left[\nabla x_{1}+\frac{\alpha x_{1} x_{2}}{T} \nabla T\right]=-c D\left[\nabla x_{1}+S_{T} x_{1} x_{2} \Delta T\right]=-c\left[D \nabla x_{1}+D_{T} x_{1} x_{2} \nabla T\right]$

where $c$ is the mixture molar density, $D$ is molecular diffusion coefficient, $x_{1}$ and $x_{2}$ are the mole fractions of components 1 and 2, respectively, $\nabla x_{1}$ is the spatial gradient of component 1 , and $\nabla T$ is the spatial temperature gradient. Eq. 2.1 is generally used in one dimension, as most experiments are designed such that the heat and mass transfer occur in one direction only. Thermodiffusion is present in situations such as liquid molten metal mixtures [17, 18], separation of polymer blends [19], particle and macromolecule manipulation [20], and underground hydrocarbon reservoirs in a porous medium [21], to name a few. 
In linear non-equilibrium thermodynamics (LNET), heat and mass (molar) fluxes are associated linearly with thermodynamic forces, such as temperature and concentration gradients [22]. In the presence of a temperature gradient within a mixture, after introducing the net heat of transport and assuming that the pressure gradient is zero both locally and globally, the molar diffusion flux of the $i^{\text {th }}$ component may be written as follows [23, 24]:

$J_{i}=\sum_{k=1}^{n} L_{i k}\left[Q_{k}^{*} \frac{\nabla T}{T}+\sum_{j=1}^{n-1} \frac{\partial \mu_{k}}{\partial x_{j}} \nabla x_{j}\right]$

where $J_{i}$ is the molar flux of component $i, T$ is the mixture temperature, $\nabla x_{j}$ is the spatial gradient of component $j, L_{i k}$ are the phenomenological coefficients, $\mu$ is the chemical potential, $Q_{k}^{*}$ is the net heat of transport of component $k$, which is the heat flow per mole of the diffusing component $k$ needed to move to the local region by mass diffusion to keep the temperature steady. In a steady or stationary state, when a non-uniform concentration profile is formed during solidification, the mass fluxes disappear while a temperature gradient and heat flow exist. Bearing in mind that for thermodiffusion, for a coupled heat and mass transfer phenomenon to be present the phenomenological coefficients $L_{i k}$ are non-zero, and one may show that the summation of all terms in the square brackets in Eq. 2.2 is zero [25]. This operation produces two equations relating $\nabla T$ and $\nabla x_{j}$. Those equations, combined with Eq. 2.1 and the GibbsDuhem relationship $\left(\sum x_{i} d \mu_{i}=0\right)$ at constant pressure and temperature, results in the thermodiffusion factor of the first component in terms of the net heat of transport [25]:

$\alpha=\frac{Q_{1}^{*}-Q_{2}^{*}}{x_{1}\left(\frac{\partial \mu_{1}}{\partial x_{1}}\right)}$ 
Eq. 2.3 is a general expression for the thermodiffusion factor. The thermodiffusion factor can be calculated through the use of two main theoretical models: the phenomenological approach (or thermostatic approach) and the kinetic approach. Both methods have been used as the basis of modeling the thermodiffusion factor in binary compositions, and both methods depend on the postulates of the phenomenological theory of non-equilibrium thermodynamics. The concept of non-equilibrium thermodynamics is extended from equilibrium thermodynamics by the introduction of the following four assumptions (Curie's postulates) [26]:

1. The equilibrium thermodynamics relations may also apply to systems that are not in equilibrium if the gradients are not too large (quasi-equilibrium postulate).

2. All fluxes in the system may be considered as having linear relations involving all the forces (linear postulate).

3. No combination of fluxes and forces occurs if the difference in order of the flux and force is an odd number (Curie's postulate).

4. When the magnetic field is not considered, the matrix of the coefficients in the flux-force relations is symmetric (Onsager's reciprocal relations).

Two common models that use the thermostatic approach are the Haase model and the Kempers model. The Haase model is based on phenomenological theory, and the net heat of transport is introduced with the partial molar enthalpy. Therefore, the net heat of transport for a binary mixture is given by [27]:

$Q_{2}^{*}-Q_{1}^{*}=\frac{M_{1} M_{2}}{M_{1} x_{1}+M_{2} x_{2}}\left(\frac{\bar{H}_{2}}{M_{2}}-\frac{\bar{H}_{1}}{M_{1}}\right)$

where $\bar{H}_{i}$ is the partial molar enthalpy of component $i$ and $M_{i}$ is the molecular weight of component i. By substituting Eq. 2.4 into Eq. 2.3, the thermodiffusion factor is expressed as [27] 
$\alpha_{T}^{H}=\frac{M_{1} \bar{H}_{2}-M_{2} \bar{H}_{1}}{\left(M_{1} x_{1}+M_{2} x_{2}\right) x_{1}\left(\frac{\partial \mu_{1}}{\partial x_{1}}\right)}$

The thermodiffusion factor for an ideal fluid at standard state is then given as [27]

$$
\alpha_{T}^{\circ}=\frac{M_{1} \bar{H}_{2}^{\circ}-M_{2} \bar{H}_{1}^{\circ}}{R T\left(M_{1} x_{1}+M_{2} x_{2}\right)}
$$

For an ideal fluid, the relation $x_{i}\left(\frac{\partial \mu_{i}}{\partial x_{i}}\right)=R T$ pertains, where $\mathrm{R}$ is the gas constant.

Winter and Drickamer [28] applied an alternate form of Eq. 2.3 following a treatment in a volume frame of reference. They noted that if the net heat of transport, which is the energy required to be absorbed by the local area to activate a molecule of type $i$ and help that molecule to jump to the next available site, is approximated by half of the volume density of the activation energy of viscous flow, a good match is obtained between the model predictions and their experimental data. Below is their semi-empirical expression for the thermodiffusion factor [28]:

$\alpha=\frac{M_{2} \overline{V_{1}}+M_{1} \overline{V_{2}}}{2 \bar{M} x_{1}\left(\frac{\partial \mu_{1}}{\partial x_{1}}\right)}\left[\frac{E_{1}^{v i s}}{\overline{V_{1}}}-\frac{E_{2}^{v i s}}{\overline{V_{2}}}\right]$

In Eq. 2.7, $M_{i}, \bar{V}_{l}$ and $E_{i}^{v i s}$ are the molecular weight, the partial molar volume and the activation energy of viscous flow of component $i$, respectively, and $\bar{M}=M_{1} x_{1}+M_{2} x_{2}$. 
In a series of studies on different liquid compositions, Eslamian and Saghir [17] followed an approach similar to that of Winter and coworkers [28]. They assumed that the activation energy of a viscous flow was equal to the net heat of transport. While this assumption is true in the case of a pure liquid system, but the activation energy might be different in the case of viscous flow of a component considered in a mixture. Note that their model is systematically valid for a number of liquid mixtures with only slight modifications. For liquid systems composed of non-associating molecules, this equation is written as follows [17]:

$$
\alpha=\frac{E_{1}^{v i s}-E_{2}^{v i s}}{x_{1}\left(\frac{\partial \mu_{1}}{\partial x_{1}}\right)}
$$

The method that the authors used to find the activation energy of viscous flow is explained in detail in [29]. The logarithmic plot of the temperature-dependent viscosity multiplied by liquid molar volume (kinematic viscosity) against $1 / R T\left(\mathrm{~J}^{-1} \mathrm{~mole}\right)$ is a line whose angle is the activation energy of viscous flow of that liquid ( $\mathrm{J} / \mathrm{mole})$. According to [17], this is the energy per mole needed to put the liquid in motion. Eq. 2.8 has shown reasonable performance while tested against the experimental data of binary linear hydrocarbon mixtures. This modeling approach, with proper modifications, has been applied on associating components as well, such as alcohol-water systems. Indeed, it is the first stand-alone model that has been able to predict a sign change in the thermodiffusion factor in associating components with varying mixture compositions [17].

The study of thermodiffusion in molten metal alloys and the theories describing it were initially under the influence of those thermodiffusion models that were derived for the 
temperature-driven migration of impurities in solid metal alloys. In solid metal alloys, the thermodiffusion analysis is based on a drift velocity for each component [31]. The drift velocity is a function of the heat of transport and the molar enthalpy change in the lattice that forms a void in the surrounding solvent atoms. However, this description is comparable to the nonequilibrium-based thermodynamic models, which describe a heat of transport and activation energies. The thermodiffusion models for solids were then revised and expanded to include molten metal alloys by other researchers such as Lodding [32]. Lodding developed the following approximate expression for the separation or enrichment factor, $Q$, which was outlined as the ratio of concentration (c) of components 1 and 2 on the hot side to that on the cold side. Note that $Q$ is derived from the net heat of transport expressed in non-equilibrium thermodynamics [32]:

$$
Q=\left(\frac{\left(\frac{c_{1}}{c_{2}}\right)_{H}}{\left(\frac{c_{1}}{c_{2}}\right)_{c}}\right)=E_{D}\left(\frac{1}{T_{C}}-\frac{1}{T_{H}}\right)\left(\frac{\phi_{1}-\phi_{2} \alpha^{\prime}}{c_{2}+c_{1} \alpha^{\prime}}\right)+1
$$

In Eq. 2.9, $E_{D}$ is the activation energy stated as the derivative of the natural logarithm of the diffusion coefficient scaled by $1 / R T, \alpha^{\prime}=D_{2} / D_{1}$ is the mobility ratio, where $D_{1}$ and $D_{2}$ are the molecular diffusion coefficients and $c_{1}$ and $c_{2}$ are the average concentration of components 1 and 2 in the composition, respectively, and $T_{C}$ and $T_{H}$ are the temperatures at the cold side and the hot side, respectively. The parameter $\phi$ is associated with the particle diameter and transposition. It is hard to estimate the performance of Eq. 2.9, as it comprises many undetermined factors.

Later, Galina and Omini [33] reconsidered Lodding’s theory and expanded it to include expressions that could be used to estimate the thermodiffusion factor in metal isotopic 
components and mixtures of different metal alloys. Unlike non-associating non-ionic liquids, such as linear hydrocarbons, liquid molten metals consist of large ions and movable, conduction electrons. They proposed that in Liquid metals, there are two forces that are exerted on the particles: a thermal force $F_{T H}$ due to a temperature gradient (which is also present in nonassociating liquid compositions such as hydrocarbons) and an electronic force due to an induced electric field $F_{E}$. The former is sometimes called the extrinsic force (impact between ions) and the latter is called the intrinsic force (electronic contact and scattering between conduction electrons and ions). In a case that conduction electrons do not exist in a liquid molten metal alloy, the ions of various components would act as insulating components composed of hard spheres. In the case of a molten metal alloy, when the composition experiences a temperature gradient, an internal electric field is induced. This is partly due to the movement of the conduction electrons and partly due to ions in the thermal field. In the Galina-Omini approach, both thermal and electronic forces were studied. They claimed that at temperatures close to the melting point of the composition, the electronic force is one order of magnitude smaller than the thermal force. However, as the composition temperature increases, the electronic force becomes larger and more important. This effect will be explored later in this thesis in chapter 3. For an isotopic mixture, Galina-Omini simplified the equations and suggested a model for the estimation of the separation factor $Q$. Their proposed model was verified by comparing the results with the experimentally measured data of thermodiffusion in isotopic mixtures of $\mathrm{Li}, \mathrm{K}$, Ga, and In. The accuracy of their model was notable, even though it is not clear how consistent their ground-based experimental data were. For binary molten metals, their equations are more complex, since numerous geometric and lattice factors were included. For a dilute solution, in 
which the concentration of one component was small, their equations were simplified and decoupled from the enhancement factor [33]:

$$
Q=\frac{L w_{2}}{D_{2}}\left(1-\frac{\gamma_{21}}{\gamma_{11}} \frac{D_{2}}{D_{1}} \frac{w_{1}}{w_{2}}\right)-\delta_{2} \Delta T\left(1-\frac{\lambda_{21}}{\gamma_{11}} \frac{\delta_{1}}{\delta_{2}}\right)+\frac{\frac{\gamma_{21}}{\gamma_{11}}-1}{1+n \gamma_{11}}\left(\frac{L w_{1}}{D_{1}}-\delta_{1} \Delta T\right)+1
$$

In Eq. $2.10, L$ is the length of the thermodiffusion cell along which a temperature difference $\Delta T$ is used, $D_{i}$ is the molecular coefficient of component $i, w_{i}$ is the drift or mobility velocity of component $i, \gamma_{i j}$ and $\delta_{i}$ are two parameters correlated to the particle average free path and the probability per unit time that a particle obtains enough energy to jump to the next available location, respectively. $\delta_{i}$ has been defined in terms of measurable parameters, but it is not straightforward to do so for $\gamma_{i j}$. For isotopes, however, there is no need to calculate $\gamma_{i j}$, as $\gamma_{11}$ $=\gamma_{21}$. Using the Lamb formula (similar to the Stokes formula in fluid dynamics) and considering ions as spheres flowing in a stream, the drift velocity $w_{i}$ was predicted as a function of viscosity $\eta$ and the ion radius $r_{i}$ as below [33]:

$$
w_{i}=\frac{1}{4 \pi \eta r_{i}} F_{T}
$$

where $F_{T}$ is the total of all forces acting on a particle or ion in a composition. Galina and Omini argued that the temperature-induced force applied to a particle in a liquid mixture is almost equivalent to the force applied to a particle in a solid lattice, assuming that the particle performs as an Einstein harmonic oscillator. This thermal force is given as $F_{T H}=-K \nabla T$, and in the absence of all other forces it causes a particle to transfer normally from the hot side of a 
temperature gradient to the cold side. In molten metal alloys, owing to the existence of the conduction electrons, an extra electronic force is applied on the ions. Galina and Omini extracted the following equation for this force [33]:

$$
F_{E}=\left\{z\left(\frac{\pi^{2} k_{B}^{2} T}{2 E_{f}}-e S\right)\left(\frac{1}{\sigma} \frac{\partial \sigma}{\partial c}\right)+e z \frac{\partial S}{\partial c}\right\} \nabla T-e \Delta z S \Delta T
$$

In Eq. 2.12, $e$ is the electron charge and is negative, $E_{f}$ is the Fermi energy, $S$ is the metal thermoelectric power, $\sigma$ is the conductivity, $\mathrm{z}$ is the valance of the solvent and solute ions, $\Delta z$ is the difference of solute valence with regards to the solvent valence, and $c$ is the solute concentration. In order to complete the Galina-Omini model, $F_{T}$ in Eq. 2.11 is defined as $F_{T}=F_{T H}+F_{E}$. Compared to the Lodding formula, the Galina-Omini model is more complete and sophisticated as theoretically it can be appropriately applied to systems with two different metal components as well. On the other hand, the assumption that the thermal forces act as $F_{T H}=-k \nabla T$ in this approach is not without controversy, and Eq. 2.12 includes many parameters that may not be easily available for binary molten metals [33].

Bhat and Swalin [34] expressed thermodiffusion in a molten metal alloy by applying nonequilibrium thermodynamics in a volume frame of reference. In their equations, alongside the typical equations such as the equation developed by Dougherty and co-workers [23], they suggested that in the steady state once the material fluxes vanish, in the continuous volume system both of the following equations instantaneously hold true: $J_{k}^{\prime}=0$ and $\sum \bar{V}_{k} J_{k}^{\prime}=0$, where $J_{k}^{\prime}$ is the molar flux and $\bar{V}_{k}$ is the partial molar volume of component $k$. Conversely, only one of the aforementioned expressions may hold at a time, subject to the frame of reference that is chosen [32]. As the modified Fick's law (Eq. 2.1) plays no role in the derivation of their 
equation, their expression takes the following form, which connects the net heat of transport of the two components to other properties of the composition, ignoring the thermodiffusion factor or coefficient [23]:

$$
\left(\frac{1}{\bar{V}_{1} x_{1}}+\frac{1}{\bar{V}_{2} x_{2}}\right) d \mu_{2}=-\frac{1}{T}\left(\frac{Q_{2}^{*}}{\bar{V}_{2}}-\frac{Q_{1}^{*}}{\bar{V}_{1}}\right) d T
$$

Subsequently, Bhat and Swalin defined the net heat of the transport of a binary system as:

$Q^{*}=Q_{2}^{*}-Q_{1}^{*}\left(\frac{\bar{V}_{2}}{\bar{V}_{1}}\right)$. Assuming an ideal dilute solution (in an ideal solution, chemical potential may be presented in terms of the mole fraction), Eq. 2.13 takes the following form for the net heat of transport in a binary system [34]:

$Q^{*}=R \frac{d \ln x_{2}}{d\left(\frac{1}{T}\right)}$

Other researchers, such as Thernquist [35] and Tyrell [36], have suggested equations similar to P. Praizey et al. [37], who followed a similar method to Bhat and Swalin [34], but in order to describe the Soret coefficient they presumed $J_{1}+J_{2}=0$ (a mass or molar frame of reference) and also $J_{1}=J_{2}=0$.

Regardless of the validity of Eq. 2.14, when the natural logarithm of the relative concentration of solute is plotted against $1 / R T$, an almost linear curve is obtained through a temperature range. The slope of that line has been studied as the net heat of transport [25]. Various researchers have successfully used Eq. 2.14 to predict the net heat of transport using the experimental data of similar systems [38]. 
The approach used for thermodiffusion in other liquid compositions is completely different than the method discussed above. This difference has created a debate and has led to confusion in the best way to model thermodiffusion in molten metal alloys. However, the net heat of transport defined by Eq. 2.14 may still be used as a measure of thermodiffusion in these systems. Other equations have also been applied to estimate the net heat of transport in additional systems, such as by A. D. Payton et al. [39]. Note that in the non-equilibrium thermodynamicbased models, the net heat of transport is a property of the mixture and is a function of the phenomenological coefficients that have to be physically derived or calculated before the thermodiffusion factors can be estimated. Therefore, for consistency, it has been accepted the classic explanation of the net heat of transport applied in non-equilibrium thermodynamics.

Inspired by Eq. 2.14, which allows for the prediction of the net heat of transport experimentally, and in an effort to improve the model to calculate the heat of transport, both Bhat and Swalin [34] and Thernquist [35] presented the net heat of transport derived in Eq. 2.14 as the sum of the electronic and ionic influences. To explain the electronic contribution, they applied Gerl's formula [40], while for the thermal contribution for dilute isotope gas mixtures Jones' formula [41] was used, and for dilute binary mixtures the model developed by Chapman and Cowling [42] was used. The formulas suggested by Jones [41] or Chapman and Cowling [42] were presented for non-uniform gases with application of kinetic theory and as such was not applicable to molten metal alloys.

In Gerl's [40] expanded theory for dilute mixtures, he considers solute particles as point charges that are affected by moving electrons. The force exerted by the conduction electrons on a solute ion is given as follows [36]: 


$$
F_{E}=\frac{K_{e}}{\sqrt{2}}\left(\frac{m^{*}}{m}\right)^{\frac{1}{2}} E_{F}^{-\frac{1}{2}} A_{Z}\left(E_{F}\right) \times\left[1+2\left(\frac{d A / A}{d E / E}\right)_{E_{F}}\right] \nabla T
$$

where $m$ and $m^{*}$ are the rest and effective masses of an electron, $E_{F}$ is the Fermi energy, $K_{e}$ is obtained by using the Shimoji's equation [43], and $A_{Z}$ is the effective cross-section of electronion scattering. While this equation is beneficial, calculation of $A_{Z}$ and the term in the square bracket is challenging. Estimating $\frac{m^{*}}{m}$ is also a complex issue, but this ratio normally varies between 1 and 1.5, according to V.K. Ratti and E. Evans et al. [44]. Bhat and Swalin [34] presented the above-mentioned parameters for gold (in a dilute mixture of silver in gold): $A_{Z}$ is a function of the derivative of the electrical resistivity of the alloy and dependent on composition. For many molten metal alloys, this derivative is not constant across the composition range, and its sign may even change. This variation in $A_{z}$ suggests that as concentration changes, the magnitude and direction of the electronic forces may change as well, as predicted in Eq. 2.15.

Later, Praizey and co-workers [37] used the Bhat and Swalin [34] and Thernquist [35] expression with the addition of the Soret coefficient in order to find a link between the Soret coefficient and the heat of transport $Q^{*}$ given by Eq. 2.14. The Soret coefficient presented by Praizey $\left(S_{T}^{\prime}\right)$ based on the solute concentration is related to the mass flux as follows (in fact this definition is valid for dilute solutions $\left(x_{1}=1-x_{2} \cong 1\right)[37]$ :

$$
j_{2}=-c D\left[\nabla x_{2}+S_{T}^{\prime} x_{2} \nabla T\right]
$$


At steady state, the mass fluxes disappear and $S_{T}^{\prime}$ (of the second component or solute) is calculated by using the following equation: $S_{T}^{\prime}=-d \ln x_{2} / d T$. Praizey correlated $Q^{*}$ in Eq. 2.14 with $S_{T}^{\prime}$ through the following expression $Q^{*}=S^{\prime} T R T_{2}$.

Praizey applied the Bhat and Swalin expression to compare experimental data with thermodiffusion theory as $Q^{*}$ in Eq. 2.14 and $S_{T}^{\prime}$ can be obtained readily from experiment. Later Praizey and co-workers [45] adopted a different approach to estimate thermodiffusion factors rather than using the controversial equation of Bhat and Swalin [34] for the net heat of transport.

In summary, the above literature review shows a lack of consensus over the modeling of thermodiffusion in liquid molten metal alloys and a debate over the proper presentation and prediction of experimental data.

\subsection{Natural Convection and Surface Radiation}

To study the external natural convection in two-dimensional enclosures, a temperature gradient is applied across two opposing walls, either vertically or horizontally. One of the most readily used configurations is one in which the bottom surface is the heat source, to induce Rayleigh-Benard convection. In this configuration, flow is generated owing to the boundary conditions at the bottom wall and the applied temperature gradient. The fluid flow and heat transfer in the cavity becomes more complex as a binary system is considered and/or surface radiation is considered as well. The presence of surface radiation on the vertical outside walls indicates that the boundaries are no longer insulated. There are numerous studies on natural convection in cavities. Here, the pertinent natural convection studies on binary systems that include radiation are reviewed. 
The relationship of natural convection with thermal radiation from gray surfaces in a square cavity and heated air enclosure was studied numerically by Balaji and Venkateshan [46, 47] and by Akiyama and Chong [48]. They conducted experimental and numerical studies in a square cavity with variable heat source and isothermal vertical walls. They considered high as well as low values of emissivity for the inner surfaces of the cavity. They successfully calculated the adiabatic horizontal boundary conditions with air as the working fluid. A purely experimental study utilizing differential interferometry was conducted by Ramesh and Venkateshan [49]. They studied heat transfer by natural convection and surface radiation in a differentially heated square cavity filled with air with highly emissive walls.

Ridouane et al. [50] numerically studied the combination of natural convection and surface radiation in a square cavity with heating from the bottom wall. They explained that the surface radiation significantly decreases the critical Rayleigh numbers, which describe the transition from steady-state convection to an oscillatory convection. They also concluded that, depending on $R a$, a transition from a steady state to a chaotic state is achievable by increasing the value of the emissivity. Recently, Gururaja Rao et al. [51] studied the problem of multimode heat transfer with three identical heat sources on a square-shaped electronic device. They looked at how the governing parameters would affect the local temperature distribution and the maximum temperature of the device. They also looked at the contribution of natural convection and surface radiation to heat dissipation from the device. Bahlaoui et al. [52] numerically studied the combined effects of natural convection and radiation in a tall rectangular cavity. They concluded that there exists multiple steady-state solutions in an inclined cavity and that the presence of radiation affects the number of solutions. They then explained that increasing the emissivity causes a reduction of the number of solutions for low values of the Rayleigh number. 
Alternatively, increase of the latter will increase the number of solutions for all the values of emissivity considered. Han and Baek [53] showed numerically the combining radiation and natural convection in a rectangular enclosure with two incomplete divider under a large temperature difference. They presented that the radiation significantly alters the fluid dynamic and temperature distribution compared with cases without surface and gas radiation, and the effect of the latter was detected to be more significant than that of the prior. Balaji and Venkateshan [46, 47], Hinojosa et al. [54], and Dehghan and Behnia [55] studied the combination of convection and surface radiation in partially opened cavities either uniformly or separately heated. They found that the radiation affects the dynamic and thermal structure of the fluid, decreases the natural convective heat transfer component, and causes an increase in the total amount of heat exchanged.

In the above studies, it was assumed that the thermal boundary conditions were either steady isothermal or constant heat flux wall conditions. However, in many engineering applications this is not the case, and the energy inputs of the system vary with time, which causes unsteady natural-convection flow. Solar collectors and printed circuit boards are good examples of such systems that experience variable thermal boundary conditions. Furthermore, it is almost impossible to predict the thermal and dynamic behaviours of fluid under a time-dependent condition from results that are collected under constant temperature or heat flux conditions.

Lage and Bejan [56] numerically and theoretically explored the problem of natural convection in an enclosure with a pulsating heat flux. Their results showed that the buoyancyinduced flow resonates with a specific frequency of the pulsating heat input. The resonance was described by the maximum fluctuations obtained in the evaluation of heat transfer resulting from the time-dependent temperature. Lakhal et al. [57] numerically obtained results for a square 
cavity heated from below with cyclic variable applied temperatures. They demonstrated that the flow structure and heat transfer are strongly coupled to the amplitude and the period of the varying temperature. Lakhal et al. [58] later expanded their work and studied the transient natural convection in a square cavity. The cavity was cooled from above and partially heated from the side with temperature changing sinusoidal as a function of time. The oscillation frequency of the temperature that resulted in the resonance phenomenon was obtained for both types of applied excitations. The resonance frequency was found to be dependent on the heating amplitude. They showed that the pulsating temperature would result in an enhanced heat transfer as compared to that of the sinusoidally varying temperature.

Antohe and Lage [59] studied the transport of momentum and heat of a clear fluid and of a fully saturated porous medium. They considered an enclosure that was subjected to varying heat with a periodic heat flux. Their result showed that the natural convection within the enclosure was such that it produced several local maxima at specific values of the heating frequency. They determined that their results were independent of the heating amplitude for both the clear fluid and porous medium cases. They introduced a theoretical correlation between the resonance frequency and the Rayleigh and Darcy numbers that provided a precise means for guiding numerical simulations; one that results in a precise determination of this resonance frequency. Later, Antohe and Lage [60] studied the influence of the Prandtl number on the natural-convection flow in an enclosure with time-dependent heating applied from the side. The analysis of the theoretical model showed that the resonance frequency of a porous medium or fluid is directly proportional to $\mathrm{Pr}^{1 / 2}$. This resonance phenomenon is considered damped if the Prandtl number is increased or decreased from a value of unity, keeping the Rayleigh and Darcy numbers constant. 
Kwak and Hyun [61] and Kwak et al. [62] also studied this resonant phenomenon in natural convection. They determined that the resonant frequency could be simply computed using the Brunt-Vaisala frequency that is produced by the thermal stratification of a system. Abourida et al. [63] also explored the case of convective heat transfer for a square cavity filled with air that was subjected to various thermal boundary conditions on its horizontal and vertical walls. They showed that at high Rayleigh numbers the periodic heating could slightly enhance or decrease the heat loss, compared to the application of steady temperatures. They also reported that in a cavity with a temperature gradient applied vertically, the buoyancy in the system caused flow. This flow led to resonance in the presence of periodic heating, but only when the cold temperature is constant. Equally, the resonance phenomenon was not observed when the hot and cold temperatures at the opposing walls were simultaneously varied.

They discussed two-dimensional transient natural convection in a horizontal channel at with heating from the bottom wall at regular intervals. In their study, the temperature was changed sinusoidally with time, and rectangular adiabatic blocks were distributed on the lower wall. Their results showed that there are different routes leading to turbulent flow. These were identified by gradually increasing and varying the amplitude of heating as well as the period of the heating. In a more recent work, Zhao et al. [64] explored, numerically and theoretically, the transient laminar natural convection that is induced by two separate heating elements attached on one vertical wall of a square cavity. One heater was used to provide the variable time-dependent temperature to the system, and the second element provided a means for maintaining a constant heating temperature. The resonance frequencies for the system were determined numerically using a theoretical calculation. The interaction between the two heat sources was examined to determine the maximum overall heat transfer rate. It was found that when the upper heater 
delivers a variable temperature, the local Nusselt number evaluated at the lower section is negligible. Conversely, when the actions of the heaters were exchanged, and the time-varying temperature control is used with the lower heating element, a time-varying local Nusselt number is induced at the location of the upper heater.

Patterson and Imberger [65], studied a rectangular cavity with an aspect ratio (height/length) $<1$ with adiabatic top and bottom walls and differentially heated side walls by using a finite difference method. They have chosen different combinations of the Rayleigh number $(R a)$, Prandtl number $(P r)$, and aspect ratios, to simulate different possible flow models. The critical Rayleigh number was defined as $R a_{c}=\max \left(\operatorname{Pr}^{2}, A^{-12}\right)$. With regards to $R a_{c}$, the heat transport can be separated into three regimes: conduction, convection, and transition. While $R a<$ 1 , heat conduction is the main effect that controls the flow in the cavity, and due to the low velocity of the fluid the isotherms are straight lines. When $R a>R a_{c}$, the flow is dominated by heat convection, and thermal boundary layers develop. The isotherm lines in this case are affected by the convective loop over the cavity. For $1<R a<R a_{c}$, the flow is a combination of conduction and convection. However, when $R a>\operatorname{Pr}^{4} A^{-4}$, the regime reaches the steady state in an oscillating pattern.

Kublbeck et al. [66] presented a computational method that describes the laminar freeconvection flow inside the cavity. They solved the energy and momentum equations by applying the alternating direction implicit method (ADI) and the cyclic reduction to the Poisson equation. In their study, two structures were examined in test runs of natural convection in a twodimensional rectangular cavity: The cavity with consistently heated and cooled sidewalls, and the cavity with the three walls that were adiabatic with one vertical wall that was heated nonuniformly. Their method was shown to be effective and accurate. Ivey [67] presented an 
experiment to study the transient flow regime that was suggested in Patterson and Imberger [65]. In their experiment, the temperature oscillated with large amplitudes for a short period of time, and they found that the flow pattern was different compared to the earlier studies.

Schladow et al. [68] performed a number of two and three dimensional numerical simulations of transient flow based on one of the experimental cases predicted by Ivey [67], which was a cavity heated via the side walls and possessing a high $R a$ of $2 \times 10^{9}, A$ of 1 , and $P r$ of 7.1. Schladow found that during the early steps of transient flow in this cavity, oscillation over both the whole cavity and on the boundary oscillations occurred. The oscillation within the cavity was produced by the horizontal pressure gradient determined by the temperature gradient, and the boundary oscillations by two kinds of instabilities at the thermal boundary layers.

Along with individual numerical, analytical, or experimental studies on natural convection in cavities, several investigators made some comparisons between numerical and experimental prediction. Patterson and Armfield [69] and Jeevaraj and Patterson [70] studied convection caused by consecutively heating and cooling opposing vertical walls with a water or glycerol-water working fluid. They showed that cavity-scale oscillations were caused by the interactions between the two opposite vertical boundary layers. Patterson and Imberger [65] confirmed for the first time that the classification of the flow regime developed in stages before reaching a steady state.

There are many successful research studied that focus on time-dependent convective flow behavior in a bottom-heated cavity. In bottom heating, the flow is very sensitive to the thermal boundary conditions, and small agitations at the boundary can extinguish the established pattern of the flow. Koster [71] investigated experimentally the local instabilities of a Hele-Shaw cell with bottom heating and top cooling. In their case, the slot in the cell was filled with silicone oil 
with a $\operatorname{Pr}$ number of 37 . The material of the four vertical walls of the slot was Plexiglass, which possesses low thermal conductivity. They used the holographic interferometry technique in the slot to image the oscillatory convective flow patterns and to find the thermal boundary layer region where the instabilities were most likely to exist. This work showed that the thermal boundary layer was a major cause of oscillatory convection. Koster and Muller [72] showed that different boundary layers might be the reason that temperature oscillations with different amplitudes and frequencies appear. Hence, the instability criterion has to be applied individually based on each boundary layer.

Kazmierczak and Chinoda [73] numerically studied laminar flow driven by buoyancy in a square cavity, with one fixed temperature vertical wall and sinusoidally varying temperature applied on the opposite wall. The governing equations were applied by using the control volume formulation. The power law scheme was used to calculate the heat and mass fluxes across the boundaries of each control volume. They concluded that the transient flows were periodic with the temperature oscillation, although the time to achieve that periodic state was highly dependent on the amplitude and the period of the surface oscillation. A primary recirculation loop was found within the cavity, and a weak secondary cell was seen at the corner of the cavity. The sinusoidal wall temperature only took effect across the thermal boundary layer. Even though the velocity and temperature distribution have a significant change near the boundaries, the average heat transfer along the cavity was not altered owing to the long heating period.

The numerical work of Abourida et al. [63] considered the transient natural convection of an air-filled square cavity. They looked at three cases in their study: In the first case, the temperature of the top wall varied sinusoidally with time and the opposite wall was kept at a steady temperature. In the second case, temperatures of both horizontal walls were periodic in 
phase. The third case was similar to second except that the temperature applied between the two walls had a phase difference of $\pi$. All other cavity walls were assumed to be adiabatic. They used a finite difference procedure in the simulation and the mesh independence was verified. They studied the velocity distributions, contours of the temperature distribution, and the rate of heat transfer for all three cases. Their results showed that flow is periodic when $R a$ is less than $10^{6}$. Therefore, the heat transfer can be increased or diminished due to various heating modes, periodic temperatures, and the $R a$.

Poujol et al. [74] worked on the transient natural convection, both numerically and experimentally, in a square cavity heated by a time-dependent heat flux on one vertical wall and constant temperature on the opposing wall. The fluid in this study was silicon oil and thermocouples were used to measure temperatures at the boundary and inside the cavity. The velocity profiles from the experimental work was visually determined and compared with the results from the numerical model. However, the results were not able to determine the magnitude of the velocity due to the low quality of the images from experiments. Higgins [75] carried out some preliminary studies on oscillating natural convection inside a rectangular cavity. Their studies showed that the flow pattern becomes unstable after only a few cycles. However, they found out that the comparisons between the experimental data and numerical simulations were unsatisfactory and require new techniques for flow visualization and data analyses.

Mahidjiba et al. [76] discovered the onset of double-diffusive convection in a porous rectangular cavity. The thermal and solute boundary conditions were utilized on the top and bottom of the cavity once the vertical walls were considered impermeable and insulated. Galerkin finite element technique was applied to solve the governing equations. They explained that the start of convection depends on many factors, such as normalized porosity, the aspect 
ratio of the cavity, solute to thermal buoyancy ratio, Lewis number, and the temperature and concentration boundary conditions. The convection in an enclosed cavity can be in steady state, transition, or oscillation depending on the Rayleigh number.

Sundaravadivelu and Kandaswamy [77] outlined double diffusive convection driven by the coupling temperature and species gradients in a square cavity, numerically. They solved the momentum, the heat, species, and continuity transport governing equations by using the alternating direction implicit method (ADI) and successive over relaxation (SOR) methods. The velocity streamline, concentration, and temperature distributions as a function of various Schmidt numbers $(S c)$ were expressed. The resulting Nusselt number shows that the heat and mass transfer rates are nonlinearly correlated with respect to the temperature gradient in the cavity. Jue and Ramaswamy [78] studied a two-dimensional cavity with thermal and solute differences in periodically modulated gravity. They conducted transient calculations of the fluid flow, heat, and mass transport. They showed two types of flow oscillations, synchronous and sub-harmonic reaction, which existed depending upon the frequencies of the g-jitter distortion. They also determined that the heat and mass transfer rates were related to the types of oscillations.

\subsection{Solidification}

Natural convection may be also present in some crystallization processes. This is the subject of the work presented in chapter 5. In the solidification of binary molten alloys, all phenomena involved in the heat transfer within a binary alloy without solidification still occur, with the addition of solidification as an additional stage of the process at the end.

The process of solidification plays a vital role in metallurgy and can determine the 
physical and mechanical properties of solid alloys. It is an important process in various industries such as welding, polymer crystallization, crystal growth, casting, energy conservation, metallurgy, and refrigeration. The majority of materials solidify upon a drop in temperature. As such they undergo a phase change from a pure liquid region to a liquid-solid two-phase region, which is also called the mushy region or mushy zone, to a completely solid phase. Therefore, solidification is comprised of three phases: The solid, mushy, and liquid phases. Flow in the mushy region plays a significant role in the final solidified microstructure. Specifically, diffusion controls the solute distribution in the liquid at a given growth rate, and therefore it determines the value of the characteristic super cooling. Numerous experimental and numerical investigations have been performed for the solidification of metal materials. Several numerical simulations were improved to include heat transfer, fluid flow, and solidification kinetics [79, 80]. Nonetheless, the knowledge about the effect of the flow on the development of the mushy region is limited. Due to this, the frontier area of solid growth is more complicated to research than the other two regions [81].

In the case of vertical solidification that forms from the bottom, the temperature is higher at the top and the liquid is densest towards the bottom. Very few computer simulations have been reported that are based on experimental data of vertical solidification. The reason for this is solely due to the lack of experimental data in existence. There is a growing need for the numerical simulation of this particular solidification, and for this reason bottom-cooled vertical solidification has been the focus of much attention both experimentally and theoretically by many researchers. Sazarin and Hellawell [82] studied channel formation in Pb-Sn and other molten metal alloys. Tewari and coworkers [83] have studied the influence of growth rate and mushy region length on the progress of macrosegregation in $\mathrm{Pb}-\mathrm{Sn}$ molten metal alloys. They 
carried out vertical solidification in a high temperature furnace by raising and lowering the furnace at different velocities in a stationary crucible. They showed that convection occurred due to solute formation ahead of a growing solid-liquid interface in $\mathrm{Pb}-\mathrm{Sn}$ molten metal alloys.

Felicelli et al. [84, 85] performed mathematical simulations of convection, macrosegregation, and freckles for the case of unidirectional solidification of Pb-Sn alloys by applying a volume-averaged, single-domain technique. Sazarin and Hellawell [82] implemented controlled laboratory experiments on Pb-Sn molten metal alloys. The details of the experimental procedure, data, and analysis have been explained by Basu and Singh [86, 87]. They performed an analysis of double-diffusive convection as well as a continuum conception based on a mathematical model. They suggested that the mushy zone is one of the most important areas to study on the mathematical modeling of convection. In their mathematical modeling, they used both isotropic and anisotropic permeability models. One important limitation to consider is that the experimental data on permeability are restricted to the center of the mushy zone.

Kawaguchi et al. [88] investigated the growth of Cadmium-Zinc-Telluride (Cd-Zn-Te) crystals by using the vertical gradient freezing (VGF) technique from a non-dilute liquid metal. They concluded that controlling the convective flow could directly affect the zinc separation in the crystal growth. Okano et al. [89-90] investigated a numerical simulation for the crystal growth of Indium-Antimonide (In-Sb) using the liquid encapsulated VGF method. Barvinschi and co-workers [90] showed a technique for the vertical solidification of molten In-Sb in a silica ampoule. They implemented various thermal conditions for the numerical simulations of velocity and temperature under normal gravity. Shemirani et al. [91] explored the simulation of the growing of large diameter single bulk crystals of silicon and germanium admixture from its melt 
using the Bridgman method. They controlled both radial and axial applied temperatures to obtain a homogeneous and uniform distribution of silicon in the solvent region.

Vynnycky and Kimura [92] simulated the solidification and phase change in the presence of convection by using both numerical and analytical techniques. Mechighel et al. [93] carried out a 2-D numerical simulation to study the velocity, temperature, and concentration distribution in the dissolution process of silicon into a germanium melt by utilizing the vertical Bridgman method. They showed that gravity has a strong effect on the shape of the interfaces and showed the transport becomes convection dominated when gravity is present. Celentano et al. [94] performed a comparative assessment of the finite volume and finite element methods in problems involving natural convection. They used a thermally combined system of the incompressible Navier-Stokes equations and evaluated the thermally induced fluid flow pattern, the modified temperature field, and the heat transfer conditions. Rady and Mohanty [95] implemented an enthalpy-porosity fixed-grid technique to study the melting and solidification of pure metals in a rectangular enclosure. They determined that during solidification, recirculation cells disappear and cells near the boundary influence the local solidification rate near the top of the cavity.

V.R. Voller et al. [96] proposed an enthalpy formulation based on a fixed-grid technique for the numerical solution of convection-diffusion. Their method was based on the representation of the latent heat of evolution and the flow in the mushy zone. Voller et al. [97-99] later expressed various ways of handling the zero solid velocities in fixed-grid enthalpy solutions of solidification in a thermal cavity. At the same time, they suggested a similar process to that used by Gartling [100]. S. Eckert et al. [101] presented directional solidification in cylindrical samples of Sn-15 wt. \% $\mathrm{Pb}$ subjected to a rotating magnetic field (RMF). They compared their 
experimental results with numerical results of the temperature and the velocity distribution in liquid phase. They showed the impact of the local flow characteristics on the formation of the mushy region.

Several related studies [102-105] discovered the substantial effect that formation of convection the liquid phase has on the kinetics of solidification and on the resulting macro- and microstructures. M. Wu et al. [106] used a mix solidification technique to simulate the interface between the liquid flow and the growing mushy zone. They referenced the effect of the melt flow for laminar flow patterns in the growing mushy region. They stated that the velocity and flow patterns close to the mushy and liquid zones play an important role in formation of the mushy zone. In their work, they modeled the turbulence in the mushy region by assuming that the turbulent kinetic energy linearly decreases with the mushy zone permeability. They verified the current mixture solidification model theoretically and experimentally [107-111]. They applied the same model to describe the growth of the solid phase thickness of a continuous-cast steel slab and compared their prediction with an experiment on the breakout shell.

A. L. Maples et al. [112] investigated alloy solidification that forms horizontally to the middle of a cavity. Their model followed the growth of the solid-liquid interaction next to the cold area. They showed that during solidification, the liquid density changes across the twophase zone. Their results showed inverse segregation at a cooled surface, macrosegregation resulting from solidification with the initial transient, and macrosegregation while the width of the two-phase area was larger than the half width of the cavity.

P.A. Nikrityuk et al. [113] numerically calculated the effect of an external magnetic field on the solidification processes of two component materials. They studied a cylindrical cell with adiabatic walls with heat applied on the top and cooling applied from the bottom. They 
concluded that the magnetic field induces a convex shape of the mushy zone with its maximum located on the z-axis. Experimental and theoretical study on the influence of external magnetic fields on the solidification process of binary metal alloys have shown that the motion of the liquid metal depends on the type of magnetic field applied to the molten metal alloy [114-117]. F. Mechighel et al. [118] performed a numerical simulation to analyze the temperature and concentration behaviour in the separation process of silicon into molten germanium. They used a simplified arrangement similar to the materials arrangement used in the vertical Bridgman growth technique. Their work showed that the transport of silicon into the melt increases with time and with a flat stable interface.

Tanai L. Marin [119] presented a finite element technique for the solidification of a free surface liquid phase using a $2 \mathrm{~mm}$ diameter liquid aluminum droplet. He used the level set technique and phase re-injection for mass conservation, and imposed movement on the free surface of the liquid droplet. He showed that his proposed model could be applied to systems with high densities, high viscosity ratios, and for materials with high latent heats of melting. Pasandideh-Fard et al. [120,121] experimentally and computationally studied the solidification of tin droplets and the heat transfer between water droplets and a hot surface. They used an axial symmetry element model and the volume of fluid methods (VOF) together with the enthalpy formulation for calculating the heat transfer of pure materials.

J. C. Heinrich et al. [122] studied the effect of thermo-solutal convection in the development of flows in a vertical solidification. They used three numerical simulations: the plane-front model, an extension of the plane-front model where they included a time independent dendritic region of a specified liquid volume fraction, and a model where the dendritic area is free to develop based on local equilibrium conditions. Their simulation predicted the correct 
subjective behaviours and followed the experimental conclusions with good accuracy. Coriell et al. [123] examined the coupled convective and morphological instabilities of a binary alloy in a vertical solidification process under the plane-front assumption. Their results for prediction of the physical properties of dilute $\mathrm{Pb}-\mathrm{Sn}$ alloys showed a convective instability of solidification velocities of less than $40 \mu \mathrm{m} / \mathrm{s}$. McFadden et al. [124] suggested a nonlinear approach using a momentum equation and heat and mass transfers to model a vertical solidification process. They assumed that the interfaces between the solid and the mushy regions and between the mushy region and the liquid were planar with a constant rate of the solidification process, a concept that originally was suggested by Nandapurkar et al. [125].

Ivaldo L. Ferreira et al. [126] proposed a 2-D extension of a one-dimensional model that was used initially for the analysis of macrosegregation of multicomponent molten metal alloys. They compared the performance of an explicit/implicit method with that of a completely implicit formulation for coupling the thermal-solutal fields. Their simulation results for two-dimensional solidification problems had good agreement with the vertical and two-dimensional experimental data. Schneider and Beckermann [127] proposed a continuum formulation of the transport equations for calculation of temperature, macrosegregation, and fluid flow patterns of multicomponent molten metal alloys during solidification. Krane and Incropera [128] applied a binary molten metal alloy solidification model to a ternary alloy to study the solidification process and to simulate the transport phenomena during casting. They utilized binary eutectic troughs, intermetallic compounds, and ternary eutectic and peritectic alloys. Boeira et al. [129] performed a macrosegregation model to explain the formation of micropores during the transient solidification of aluminum alloys. They compared numerical macrosegregation patterns and porous volumetric fractions with published experimental results. Voller et al. [130] studied the 
application of a numerical scheme for coupling temperature and concentration fields in a general solidification model.

D. V. Alexandrov and D. L. Aseev [131, 132] showed analytically the influence of a temperature-varying diffusion coefficient on the unidirectional solidification of a molten binary alloy with a quasi-equilibrium mushy zone. They presented the concentration fields in the liquid, solid, and mushy phases. They also determined the rate of solidification and mushy zone thickness as a function of all thermophysical parameters. Alexandrov [133] used an exact analytical scheme to study the solidification of a binary melt possessing a mushy region.

P. A. Nikrityuk et al. [134] numerically studied the fluid flow in a binary alloy of (Pb-Wt. $85 \%$ Sn) during directional solidification. They performed the study in the presence of convection induced by a rotating magnetic field. They concluded that there are specific fluid flow phases. They reported that the secondary flow velocity surpasses the velocity of the solidification front, thus leading to a convex-shaped mushy zone.

S. Van Vaerenbergh et al. [135] applied linear stability analysis to study the influence of a temperature-dependent solute diffusion coefficient on the morphological stability during solidification of a binary alloy. Their simulation showed for an Sn-Ag alloy, the diffusion coefficient linearly depends on temperature. They further showed that the temperature dependence of the diffusion coefficient causes great variation in the wavelength at the beginning of instability.

A. K. Singh, et al. [136] investigated the role of convection during solidification using a mathematical model. They simulated the influence of different mushy zone models on convection and macrosegregation numerically and compared the results with experimental values. They showed the role of material properties on double diffusive convection by 
performing a comparative study of solidification of aqueous ammonium chloride, Fe-C, and $\mathrm{Pb}$ Sn binary system. S. D. Ridder et al. [137] used a coupled theoretical and experimental study of convective heat and fluid flow in liquid molten metals and their segregation in an axisymmetric cavity. They found that flow ahead of the liquidus isotherm to the interdendritic fluid flow is responsible for macrosegregation in the mushy zone.

The surface radiation, and natural convection haven’t been considered previously and here their effects have been overlooked. In addition, the need for a more accurate calculation of thermodiffusion factor for solidification simulation has been outlined through out the literature review. Therefore, the research work presented her focuses on addressing the effect of surface radiation to ambient and natural convection as well as the effect of thermodiffusion on solidification of binary molten metals. 


\section{CHAPTER THREE}

\section{Theoretical Approach for the Estimation of the Thermodiffusion Factor}

\subsection{Introduction}

This chapter presents an overview of the theory of the relevant mathematical models and proposes an expression for the estimation of the thermodiffusion factor in binary molten metal alloys. Specifically, the expression for calculating the thermodiffusion factor for binary molten metal mixtures was derived in detail. The derivation was based on Eslamian and Saghir's model [17] and uses Maier's approach [138] and the transition state theory to account for the chemical potential effects.

The performance of the proposed expressions on some binary molten alloys was examined and results were satisfactory. Therefore, it was followed a similar approach and developed an expression for the thermodiffusion factors for liquid metal alloys. The new formula was derived with this method accurately predicts the experimental data with greater accuracy than other existing methods.

\subsection{Model Development and Derivation}

In this work, thermodiffusion in liquid metal alloys was considered as a phenomenon consisting of two parts: ion-ion contacts known as the extrinsic or ionic contribution, and electron-ion interactions known as the intrinsic or electronic contribution. It was noted that Eq. 2.1, which was derived using the principles of linear non-equilibrium thermodynamics, only accounts for the bulk contacts between molecules in a liquid mixture in which only temperature 
and concentration gradients are present. The net heat of transport in Eq. $2.1 Q_{k}^{*}$ is therefore defined for such a system. Therefore, this equation does not appropriately describe liquid molten metal alloys. Due to this limitation, it is sensible to define a new net heat of transport, $q_{k}^{*}$ to include the presence of an induced electric field in a liquid metal mixture. This term accounts for the amount of heat required to be absorbed or released by the system in order to keep the local temperature constant. This new term results in the following modified phenomenological equation for the diffusive molar flux of component $i$ in a liquid metal alloy [34]:

$$
J_{i}=\sum_{k=1}^{n} L_{i k}^{\prime}\left[\left(Q_{k}^{*}+q_{k}^{*}\right) \frac{\nabla T}{T}+\sum_{j=1}^{n-1} \frac{\partial \mu_{k}}{\partial x_{j}} \nabla x_{j}\right] \quad i=1,2 \ldots n
$$

where $L_{i k}^{\prime}$ are the modified phenomenological coefficients. In a steady-state condition where the mass fluxes in the mixture vanish, the terms in the square brackets equal zero and Eq. 3.1 reduces to that of simple liquid mixture [34]. The resultant equations combined with Eq. 3.1 and the Gibbs-Duhem equation at constant pressure and temperature $\left(\sum x_{i} d \mu_{i}=0\right)$, lead to the following expression for the thermodiffusion factor of component 1 in a liquid metal alloy [17]:

$\alpha=\frac{Q_{1}^{*}-Q_{2}^{*}}{x_{1}\left(\frac{\partial \mu_{1}}{\partial x_{1}}\right)}+\frac{q_{1}^{*}-q_{2}^{*}}{x_{1}\left(\frac{\partial \mu_{1}}{\partial x_{1}}\right)}$

As the next step towards developing an expression for the estimation of the thermodiffusion factor, the net heat of transport was modeled by Eslamian et al. using Eq. 3.2 [17]. 


\subsubsection{Ion-Ion Net Heat of Transport}

In thermodiffusion, the structural components of the mixture such as molecules or atoms need some energy to move away from their neighbours and jump to the next existing spot. The necessary energy for this transition can be considered as equal to the net heat of transport $Q_{k}^{*}$. Flow of a viscous fluid and mass transfer in thermodiffusion may be considered similar but not identical. In both phenomena, there is an activation energy required to overcome the cohesive energy between particles and put them in motion. Hence, it is rational to consider the net heat of transfer of each component to be equal to the activation energy of viscous flow of the same component in the mixture [30]. However, the activation energy of viscous flow of a pure component is usually considered instead of the net heat of transport for the component in the mixture, since the activation energy of viscous flow for a component in a mixture is not defined [31]. Several researchers have mentioned the development of several parts of this issue; however, de Groot [6], Prigogine [140], Eyring [141], and Drickamer [28] have had the most substantial contributions to the formal development of the theory. Modeling using these assumptions has been the most popular modeling approach in thermodiffusion for numerous liquid mixtures, even though the inherent simplifications in non-equilibrium thermodynamic theory are not fully justified for this application [29]. Recently, instead of the activation energy of viscous flow, Madariaga et al. [141] directly associated the thermodiffusion coefficient with the mixture viscosity and found reasonable agreement with the experimental data.

A liquid metal alloy, although composed of near-spherical atoms, is still considered a complex mixture, owing to the presence of conduction electrons. The complexity of a mixture is determined by its behaviour during mixing and how the mixture properties compare to the properties of the individual components. Viscosity is one such property. In associating mixtures, 
a relation was found between the irregular (nonlinear) behaviour of mixture viscosity for a component and a sign change in the thermodiffusion factor [30]. In components where the mixture viscosity varies linearly with composition, no sign change for the thermodiffusion factor was observed, whereas in nonlinear systems, a crossover in migration of composition occurred.

In liquid molten metal alloys, there is no general trend describing the changes in shear viscosity with composition. Viscosities in simple liquid alloys, for instance $\mathrm{Pb}-\mathrm{Sn}$ and $\mathrm{Pb}-\mathrm{Bi}$, differ almost linearly with concentration. However, in multiple mixtures, such as Hg-Na and Hg$\mathrm{K}$ that have large negative excess enthalpies of mixing, the variation in viscosity with composition is not linear. Furthermore, in some alloys, such as $\mathrm{Ga}-\mathrm{Bi}$, the variation in viscosity changes significantly near to a critical point in the mixture composition [35]. It is predictable that a relationship exists between the sign of the thermodiffusion factor due to ion-ion impacts and variation in the viscosity of liquid metal alloys, similar to the relationship in associating mixtures.

\subsubsection{Electronic Net Heat of Transport}

The electronic net heat of transport per mole of component $i$ is correlated to the force acting on a solute ion by conduction electrons through the following equation [31]:

$$
q_{i}^{*}=F_{E}^{i} N \frac{T}{\nabla T}
$$

where $N$ is Avogadro's number. As mentioned previously, several investigators, such as Omini [33] and Gerl [40], have suggested expressions to describe this force. However, these expressions are empirical in nature and require the determination of several factors before they can be used. In a series of studies on thermodiffusion and electron movement, using statistical 
mechanics, Jones and co-workers obtained an expression for the role of electronic or intrinsic forces on the overall thermodiffusion in liquid metal alloys. Wherever a temperature gradient is applied through a mixture, they determined the following equation for the net force that is acting on one ion of type $i$ by the conduction electrons in the composition [38]:

$F_{E}^{* i}=-|e|\left(z S_{i}-z_{i} S\right) \nabla T$

where $|e|$ is the absolute value of an electron charge, $z$ is the mean valence of the ions in the mixture, $z_{i}$ is the valence of ion $i$, and $S_{i}$ is the thermoelectric power of ions of type $i$. A linear mixing rule is the simplest approximation to attain the total thermoelectric power [38]:

$$
S=\sum x_{i} s_{i}
$$

In Eq. 3.4, the magnitude of all parameters may be promptly calculated. Nevertheless, the thermoelectric power of some liquid metal alloys and their resistivity may vary linearly with changes in the composition, and, therefore, Eq. 3.5 may not be suitable for all metal mixtures. The thermoelectric power of a pure liquid metal might be obtained by employing the following equation [142]:

$S_{i}=-\frac{\pi^{2} k_{B}^{2} T}{3|e| E_{f}} \xi_{i}$ 
where $\xi$ is a dimensionless factor and is only mildly weakly dependent on temperature. Values of $\xi$ are derived from [142], which were inferred from their thermoelectric power $\left(E_{F}\right)$ [143]. The Fermi energies [144] and $\xi$ values used in the current study are provided in Table 3-1.

Table 3 - 1: Fermi energy and thermoelectric power parameter of selected molten metals.

\begin{tabular}{lll}
\hline Metal & $E_{F}(\mathrm{eV})$ & $\xi$ \\
\hline Tin $(\mathrm{Sn})$ & 10.2 & 0.4 \\
\hline Bismuth (Bi) & 9.90 & 0.55 \\
\hline Cadmium (Cd) & 7.47 & -0.2 \\
\hline Zinc (Zn) & 9.47 & -0.3 \\
\hline Lead (Pb) & 9.47 & 2.1 \\
\hline Gallium (Ga) & 10.4 & 0.55 \\
\hline Sodium (Na) & 3.24 & 2.8 \\
\hline Potassium (K) & 2.12 & 3.5 \\
\hline
\end{tabular}

Combining Eqs. 3.3 and 3.4 produce the following expression for the molar electronic net heat of transport of component 1 with respect to component 2 :

$q_{1}^{*}-q_{2}^{*}=-|e|\left(z S_{1}-z_{1} S\right) T$

\subsection{Proposed Formula}

Eslamian et al. [17] expressed a formula for the thermodiffusion factor of the first component in a liquid metal alloy. They used the activation energy of viscous flow in the pure state instead of the net heat of transport of each component owing to the ion-ion contacts, i.e., $Q_{i}^{*}=E_{i}^{v i s}$. Using Eq. 3.7 to provide the net heat of transport due to the electronic forces, Eq. 3.2 
was transformed into the next expression and was used to evaluate the thermodiffusion factor in several liquid metal alloys [17]:

$\alpha=\frac{E_{1}^{v i s}-E_{2}^{v i s}}{x_{1}\left(\frac{\partial \mu_{1}}{\partial x_{1}}\right)}+\frac{-|e|\left(z S_{1}-z_{1} S\right) T N}{x_{1}\left(\frac{\partial \mu_{1}}{\partial x_{1}}\right)}$

Maier et al. [138] also derived a theoretical model for calculation of thermodiffusion factors for binary molten metals by describing the heat of transfer by using of the latent heat of fusion, which can be found for many materials in thermodynamic databases. With the thermodynamics of irreversible processes and the theory of Onsager, the flux of component $i$ in a composition of $n$ components can be described based on at constant pressure and in the absence of external forces in the barycentric coordination [138]:

$J_{i}=\sum_{k=1}^{n-1} L_{i k}\left(-\sum_{j=1}^{n-1} \frac{\partial\left(\mu_{k}-\mu_{n}\right)}{\partial C_{j}} \nabla C_{j}-\left(Q_{k}^{*}-h_{k}+h_{n}\right) \frac{\nabla T}{T}\right)$

Here, $L_{i k}$ are the phenomenological constants, $C_{j}$ is the mass fraction of component $j, h_{k}$ is the partial specific enthalpy of component $k$, and $\mu_{k}$ the partial specific chemical potential of component $k . Q_{k}^{*}$ is defined as the heat of transfer, which is the heat transported through a specified reference plane at a constant temperature per unit of mass of compound $k$. For two components, with the help of the Gibbs-Duhem equation $C_{1} d \mu_{1}+C_{2} d \mu_{2}=0$ and $C_{1}+C_{2}=1$ at constant $T$ and $P$, Eq. 3.9 becomes [145]: 


$$
J_{1}=-J_{2}=L_{11}\left(-C_{2}^{-1} \frac{\partial \mu_{1}}{\partial C_{1}} \nabla C_{1}-\left(Q_{1}^{*}-h_{1}+h_{2}\right) \frac{\nabla T}{T}\right)
$$

They measured a microscopic section of the fluid, which was made up of one half of component 1 and one half of component 2 by mass. The average specific internal energy was then $u=$ $\frac{1}{2}\left(u_{1}+u_{2}\right)$, and the average specific volume $v_{m s p e c}=\frac{1}{2}\left(v_{1}+v_{2}\right)$ with $u_{k}$ and $v_{k}$ as the partial specific internal energy and the partial specific volume of component $k$, respectively. When the atoms of component 1 in this volume were transferred to the next equilibrium location, an amount of energy $Q_{1}^{*} d C_{1}$, a result of $Q^{*}$, will be transported by the diffusion flow. Since it was assumed that temperature and pressure were constant, a quantity of heat $d q$ had to be added and a total work of $P v_{1} d C_{1}$ had to be completed. Once a mass fraction $d C_{1}$ of component 1 was transported to the next equilibrium location, the same amount of component 2 by mass was transported from this location to the old equilibrium location of component 1 . Thus there is extra work $\mathrm{Pv}_{2} d C_{2}$ done. As a result, the change of the internal energy can then be expressed as [138]:

$$
d u=\frac{1}{2}\left(-u_{1} d c_{1}+u_{2} d c_{2}\right)=-\frac{1}{2} Q_{1}^{*} d C_{1}+d q+\frac{1}{2}\left(p v_{1} d C_{1}-P v_{2} d C_{2}\right)
$$

At this point, u, $Q^{*}, P, v$ and $d q$ are the partial specific internal energy, the specific heat of transfer, the pressure, the partial specific volume, and the heat attracted of the position of the fluid, respectively. With $h=u+p v$, the heat $d q$ added in this section of the fluid is as follows [138]:

$$
d q=\frac{1}{2}\left[\left(Q_{1}^{*}-h_{1}\right) d C_{1}+h_{2} d C_{2}\right]
$$


Hence, the authors balance the second term of Eq. 3.10 by a coefficient of $\frac{1}{2}$ and get finally

$$
J_{1}=-J_{2}=L_{11}\left(-c_{2}^{-1} \frac{\partial \mu_{1}}{\partial C_{1}} \nabla C_{1}-\frac{1}{2}\left(Q_{1}^{*}-h_{1}+h_{2}\right) \frac{\nabla T}{T}\right)
$$

to obtain the thermodiffusion factor. It was stated that the coefficient of $\nabla C_{1}$ and the coefficient of $\nabla T$ of Eq. 3.12 equal to $-\rho D_{12}$ and $\rho D_{12}^{T} C_{1} C_{2}$, respectively. $D_{12}$ and $D_{12}^{T}$ are the molecular diffusion coefficient and the thermodiffusion of component 1 in component 2, respectively [145]:

$\alpha=\frac{D_{12}^{T} T}{D_{12}}=\frac{1}{2} \frac{Q_{1}^{*}-h_{1}+h_{2}}{C_{1} \frac{\partial \mu_{1}}{\partial C_{1}}}$

To discover a physical description of the heat of transfer, the model of Garai has been used for the physical equivalent of the latent heat of fusion. This would mean that the latent heat of fusion is the energy required to weaken the viscous drag, and it would also be governed by the molar volume of the fluid, $V_{\text {mol }}[146]$ :

$L_{f}=\frac{V_{m o l} \eta v_{t h e r m}}{(n+1)^{2} d}$

where $L_{f}, V_{\text {mol }}, v_{\text {therm }}, d, \eta$ and $n$ are the molar latent heat of fusion, the molar volume of the melt, the thermal velocity of the atoms, the atomic distance, the viscosity, and the number of coupled atoms, respectively. While the authors considered a microscopic portion of the fluid 
where component 2 is replaced with component 1 by a diffusional flow as explained above, they did examine an average molar volume of this area:

$V_{m m o l}=\frac{1}{2}\left(V_{1}+V_{2}\right)$

The latent heat of fusion of component 1 was then increased by the fraction of the average molar volume to the molar volume of component 1 . For that reason, the additional energy transferred with component 1 , which is $Q_{1}^{*}$, is the difference between of the latent heat of fusion of component 1 in the average molar volume of the composition and the latent heat of fusion of the pure component $1[138]$ :

$Q_{1}^{*}=\frac{L_{f}}{m_{l}}\left(\frac{\frac{1}{2}\left(V_{1}+V_{2}\right)}{V_{1}}-1\right)$

Here, $m_{l}$ is the molar mass of component 1 . The latent heat of fusion was found by the difference of the entropy of the liquid and the entropy of the solid at the melting point multiplied by the melting temperature. Generally, transport phenomena have to be explained at a different temperature than the melting temperature, so the authors used the difference of the entropy of the liquid and the entropy of the solid at a new temperature multiplied by that temperature. They then used the FactSage database for computation of the entropy of the liquid and solid state, where the thermodynamic data is extracted by extrapolated polygons. It is conceivable that the temperature of the transport process is higher than the melting temperature of one of the pure components. This situation would mean that the entropy of the solid state of this component at the process temperature could be estimated by the extrapolated entropy function derived from the 
database, as the solid state of this component physically does not exist. Therefore, the heat of transfer develops as [138]:

$Q_{1}^{*}=\Delta S_{l}^{l s}(T) T \frac{V_{m m o l}-V_{m o l l}}{V_{m o l l}}$

Here $S_{l}^{l s}(T)$ is the partial specific entropy variation between solid and liquid state of component 1 at temperature $T$. They then obtained the derivative of the chemical potential of component 1 with reference to $C_{1}$ for dilute compositions in units of mass with $C_{1}+C_{2}=1$ and mass fraction $C_{i}$ as below [138]:

$\frac{\partial \mu_{1}}{\partial C_{1}}=\frac{\partial\left(\frac{R}{m_{1}} \ln \left(\frac{C_{1} \rho_{1}}{C_{1} \rho_{1}+C_{2} \rho_{2}}\right)\right)}{\partial C_{1}}=\frac{R T V_{2} m_{1}}{C_{1}\left(C_{1} V_{1} m_{2}+V_{2} m_{1}-V_{2} m_{1} C_{1}\right)}$

where $\rho_{i}$ is the density of component $i$ and $R$ is the gas constant. The ultimate equation of the thermodiffusion factor is then produced as follows [138]:

$\alpha=\frac{1}{2} \times \frac{\left(\Delta S_{l s}(T) T \frac{V_{m m o l}-V_{m o l l}}{V_{m o l l}}-h_{1}+h_{2}\right)\left(C_{1} V_{1} m_{2}+V_{2} m_{1}-V_{2} m_{1} C_{1}\right)}{R T V_{2} m_{1}}$

Based on the earlier discussion, it was formulated a practical expression for the thermal diffusion factor of the first component in a liquid metal alloy by introducing the new chemical potential given by Eq. 3.19 into Eq. 3.8: 


$$
\alpha=\frac{E_{1}^{v i s}-E_{2}^{v i s}}{\left(\frac{R T V_{2} m_{1}}{C_{1}\left(C_{1} V_{1} m_{2}+V_{2} m_{1}-V_{2} m_{1} C_{1}\right)}\right)}+\frac{-|e|\left(z S_{1}-z_{1} S\right) T N}{\left(\frac{R T V_{2} m_{1}}{C_{1}\left(C_{1} V_{1} m_{2}+V_{2} m_{1}-V_{2} m_{1} C_{1}\right)}\right)}
$$

Eq. 3.21 has two terms on the right hand side that may have the same or opposite signs. This equation has been derived in the framework of non-equilibrium thermodynamics. The ionion thermal and electronic forces may act on the ion of type $i$ in the same or opposite directions depending on metal alloy characteristics. For the purpose of comparison, the results of the proposed expression were compared with those of the expression derived by Eslamian et al. [17], as well as some experimental data $[45,46]$. This comparison has shown that the proposed thermodiffusion factor agrees well with the experimental results (see section 3.5).

In order to acquire the ion-ion contribution in Eq. 3.21, the activation energy of the viscous flow of metals in the pure state was obtained by using the liquid metal viscosity and density or molar volume data at different temperatures (shown in Table 3-2).

Table 3 - 2: Activation energy of viscous flow of some molten metals at different temperatures.

\begin{tabular}{lcc}
\hline Metal & Temp. $\left({ }^{\circ} \mathrm{C}\right)$ & $E_{i}^{\text {vis }}(\mathrm{J} /$ mole $)$ \\
\hline Tin $(\mathrm{Sn})$ & 295 & 560 \\
\hline Bismuth (Bi) & 295 & 7863 \\
\hline Cadmium (Cd) & 375 & 6737 \\
\hline Zinc $(\mathrm{Zn})$ & 500 & 22702 \\
\hline Lead $(\mathrm{Pb})$ & 450 & 8156 \\
\hline Gallium $(\mathrm{Ga})$ & 295 & 3915 \\
\hline Sodium $(\mathrm{Na})$ & 200 & 5648 \\
\hline Potassium $(\mathrm{K})$ & 200 & 5058 \\
\hline
\end{tabular}

The viscosity data of some metals were acquired from the following sources: tin from [147], mercury from [148], lead from [149], bismuth and zinc from [150], gallium from [151], 
cobalt from [152], viscosity and density of cadmium from [153], and viscosity of the rest of the metals were taken from the CRC handbook of chemistry and physics [154]. The measured molar volume or density data of pure metals were extracted from [155-158]. While it is preferable that the viscosity data is available close to the composition mean temperature, It is not always possible due to either to the absence of experimental data or because one of the components in the pure state is not in the liquid phase at the composition temperature. In these cases, the activation energies of the viscous flows were derived at a temperature somewhat higher than the component melting points. This issue introduces additional uncertainties while applying Eq. 3.21 for calculating the thermodiffusion factor.

Note that for ion-ion contact, i.e., the first term on the right hand side of Eq. 3.21, further expressions that have been suggested for the evaluation of the thermodiffusion factor in liquid molten metals may be applied. Regardless, some of these expressions are not obviously prescribed for metal alloys. Here, for the purpose of comparative study, our proposed model, the Winter-Drickamer [28], Haase [27], Kempers [159], Shukla-Firoozabadi [160], and Eslamian [17] formulations were tested against the experimental data (Eq. 3.21). The shortened versions of the Haase and Kempers formulas (neglecting the kinetic influence and also the enthalpies at the initial state) and the Shukla-Firoozabadi expression (for a constant matching parameter equal to 4) are written as follows:

$$
\begin{aligned}
\text { Winter-Drickamer } & \alpha & =\frac{M_{2} \bar{V}_{1}+M_{1} \bar{V}_{2}}{2 \bar{M} x_{1}\left(\frac{\partial \mu_{1}}{\partial x_{1}}\right)}\left[\frac{E_{1}^{v i s}}{\bar{V}_{1}}-\frac{E_{2}^{v i s}}{\bar{V}_{2}}\right] \\
\text { Haase } & \alpha & =\frac{M_{1} \bar{H}_{2}-M_{2} \bar{H}_{1}}{\left(M_{1} x_{1}+M_{2} x_{2}\right) x_{1}\left(\frac{\partial \mu_{1}}{\partial x_{1}}\right)}
\end{aligned}
$$


Kempers

$$
\alpha=\frac{\bar{V}_{1} \bar{H}_{2}-\bar{V}_{2} \bar{H}_{1}}{\left(\bar{V}_{1} x_{1}+\bar{V}_{2} x_{2}\right) x_{1}\left(\frac{\partial \mu_{1}}{\partial x_{1}}\right)}
$$

Shukla-Firoozabadi $\quad \alpha=\frac{\bar{V}_{1} \bar{V}_{2}}{\bar{V} x_{1}\left(\frac{\partial \mu_{1}}{\partial x_{1}}\right)}\left[\frac{\bar{U}_{1}}{4 \bar{V}_{1}}-\frac{\bar{U}_{2}}{4 \bar{V}_{2}}\right]$

Note that Haase and Kempers consider the equilibrium properties of the composition and are most appropriate for liquid molten metals close to the critical points as mentioned by Kempers [159].

\section{Equation of State (EOS)}

A comprehensive equation of state is not available for liquid molten metal alloys. This is due to the complex thermodynamics of such a composition. However, a perturbed hard-sphere equation of state (EOS) might be used for density estimation of most liquid molten metal alloys. The combination of this EOS with some correlations offers a set of equations that may be used to evaluate the molar properties of liquid molten metal alloys required to estimate the performance of Eqs. (3.22) to (3.25).

\section{Fundamental Equations}

Here an EOS is described that may be used for the evaluation of density in single or multicomponent liquid molten metal alloys. In a perturbed hard-sphere EOS, such computations usually make use of the hard-sphere fluid as a reference system, and the impact of attractions and the smoothness of revulsions are studied as perturbations in statistical-mechanical theories of fluids. However, for the smoother core potentials, like metals in liquid phases, the reference part is shaped by a set of freely jointed tangent hard-spheres [161]. Thus, the perturbed hard-sphere EOS for pure fluids was drafted as follows [162]: 
$\frac{P}{\rho K T}=1+b \rho g\left(d^{+}\right)-\frac{a \rho}{K T}$

where $P$ is the pressure, $\rho$ is the molar density, $d$ is the hard-sphere diameter, $g\left(d^{+}\right)$is the pair radial distribution function of hard-spheres at contact and $k T$ is the thermal energy per one molecule. The following analytical formulation may be applied for $g\left(d^{+}\right)$[163]:

$g\left(d^{+}\right)=\frac{1-\zeta / 2}{(1-\zeta)^{3}}$

Here $\zeta$ is the packing fraction described as $\zeta=0.25 b \rho$. Parameter $a$ in Eq. (3.24) is a measure of the strength of the attractive forces between spheres, and $b$ is the van der Waals co-volume that may be measured in terms of the appropriate reducing constants [164]:

$$
\begin{aligned}
& a(T)=\frac{2 \pi}{3} \sigma^{3} \varepsilon F_{a}\left(\frac{K T}{3}\right) \\
& b(T)=\frac{2 \pi}{3} \sigma^{3} \varepsilon F_{b}\left(\frac{K T}{3}\right)
\end{aligned}
$$

where $\varepsilon$ is the depth of minimum on potential energy curve, $\sigma$ is the distance of zero potential, $F_{a}$ and $F_{b}$ are universal functions of the decreased temperature, $k T / \varepsilon$, and can be empirically expressed as follows [163]:

$F_{a}\left(\frac{K T}{\varepsilon}\right)=a_{1} \exp \left(-a_{2} \frac{K T}{\varepsilon}\right)+a_{3} \exp \left[-a_{4}\left(\frac{K T}{\varepsilon}\right)^{3 / 2}\right]$ 


$$
F_{b}\left(\frac{K T}{\varepsilon}\right)=b_{1} \exp \left(-b_{2} \frac{K T}{\varepsilon}\right)+b_{3} \exp \left[-b_{4}\left(\frac{K T}{\varepsilon}\right)^{3 / 2}\right]
$$

The coefficients $a_{i}$ and $b_{i}$ have been regulated by fitting the EOS predictions with density experimental data and were found in [165].

To expand the proposed model to liquid molten metal alloys, the equation for pure metals can be written as follows [166]:

$$
\frac{P}{\rho K T}=1+\rho \sum_{i j}^{n} x_{i} x_{j} b_{i j} g_{i j}\left(d_{i j}^{+}\right)-\frac{\rho}{K T} \sum_{i j}^{n} x_{i} x_{j} a_{i j}
$$

where $x_{i}$ and $x_{j}$ are the mole fractions of component $i$ and $j$, respectively, $n$ is the number of components in the composition, and $g_{i j}\left(d_{i j}^{+}\right)$is the pair radial distribution function of hard-sphere composition, which takes the pattern of the Boublik-Mansoori-Carnahan-Starling (BMCS) equation [166]:

$g_{i j}\left(\eta, \xi_{i j}\right)=\frac{1}{1-\eta}+\frac{3}{2} \frac{\xi_{i j}}{(1-\eta)^{2}}+\frac{1}{2} \frac{\xi_{i j}^{2}}{(1-\eta)^{3}}$

where

$\eta=\frac{\rho}{4} \sum_{i}^{n} x_{i} b_{i}$

$\xi_{i j}=\left(\frac{b_{i} b_{j}}{b_{i j}}\right)^{1 / 3} \frac{\rho}{4} \sum_{k}^{n} x_{k} b_{k}^{2 / 3}$ 
The $b_{i j}(T)$ in Eq. (3.35) has been considered as follows:

$b_{i j}(T)=\frac{2 \pi}{3} a_{i j}^{3}(T)=\frac{1}{8}\left(b_{i}+b_{j}\right)$

$a_{i j}(T)$, which defines the attractive forces between components $i$ and $j$, has the following form:

$a_{i j}(T)=\frac{2 \pi}{3} \sigma_{i j}^{3} \varepsilon_{i j} F_{i j}\left(\frac{K T}{\varepsilon_{i j}}\right)$

where

$F_{i j}\left(\frac{K T}{\varepsilon_{i j}}\right)=\sqrt{F_{a}\left(\frac{K T}{\varepsilon_{i}}\right) F_{b}\left(\frac{K T}{\varepsilon_{j}}\right)}$

For binary liquid molten metal alloys, the geometric mean for potential well depth, viz.

$\varepsilon_{i j}=\sqrt{\varepsilon_{i} \varepsilon_{j}}$ and arithmetic mean for the potential minimum location, viz. $\sigma_{i j}=\left(\sigma_{i}+\sigma_{j}\right) / 2$ may be applied. Using the above-mentioned method, it was obtained the density of selected molten metal alloys at provided concentrations and temperatures. The density data are used in the next section to calculate the partial molar volumes of the components in the alloys. 


\section{Partial Molar Properties}

Using the EOS illustrated above, partial molar volumes, $\bar{V}_{i}, i=1,2$ may be determined in terms of the slope of the curve $V_{m}$ plotted as a function of $x_{i}$ along the isotherm-isobar $\left(\frac{\partial V_{m}}{\partial x_{i}}\right)_{P, T}$ [167]:

$$
\begin{aligned}
& \bar{V}_{i}=V_{m}-x_{i}\left(\frac{\partial V_{m}}{\partial x_{i}}\right)_{P, T} \\
& \bar{V}_{j}=V_{m}-\left(1-x_{i}\right)\left(\frac{\partial V_{m}}{\partial x_{i}}\right)_{P, T}
\end{aligned}
$$

where $V_{m}$ is the composition molar volume and is the reciprocal of the composition density achieved in the previous part. It should be noted that the perturbed hard-sphere EOS can be applied only to define the liquid densities. As a result, for theoretical calculation of partial molar enthalpies $\bar{H}_{i}$ the molecular interaction volume model (MIVM) was used, which is a twoparameter model and is capable of predicting some component activities in liquid molten metal alloys and solid solutions $[168,169]$. In agreement with the MIVM, one can get the partial molar mixing enthalpy of component $i$ in a multicomponent mixture $\Delta \bar{H}_{i}^{m}$ from the below equation [170]:

$$
\frac{\Delta \bar{H}_{i}^{m}}{R T}=-\frac{1}{2}\left[\frac{Z_{i} \sum_{j}^{n} x_{j} B_{j i} \ln B_{j i}}{\sum_{l}^{n} x_{l} B_{l i}}+\sum_{j}^{n} \frac{Z_{j} x_{j} B_{i j}}{\sum_{l}^{n} x_{l} B_{l i}}\left(\ln B_{i j}-\frac{\sum_{t}^{n} x_{t} B_{t j} \ln B_{t j}}{\sum_{l}^{n} x_{l} B_{l i}}\right)\right]
$$


where $Z_{i}$ and $Z_{j}$ are the adjacent molecules or the first coordination numbers and $B_{i j}$ and $B_{j i}$ are the pair-potential energy interaction parameters estimated by fitting for the infinite dilution mixing enthalpies of the binary liquid molten metal alloys [171].

There is another thermodynamic property, $\partial \mu_{i} / \partial x_{i}$, in the formulation presented here for the estimation of the thermodiffusion factor. This other property is known from reliable estimation models of statistical mechanics. The equations for the activities $a_{i}^{\prime}$ of liquid compositions are required for the calculation of this new term [171]:

$$
\mu_{i}-\mu_{i}^{0}=R T \ln a_{i}^{\prime}=R T \ln \left(\gamma_{i} x_{i}\right)
$$

To estimate the magnitude of $\partial \mu_{i} / \partial x_{i}$, the expression of the activity coefficient, $\gamma_{i}$ in MIVM theory was applied, which is suitable to all binary molten metal alloys used here except the Na-K alloy [171]:

$$
\begin{aligned}
& \ln \gamma_{i}= \\
& 1+\ln \frac{V_{m i}}{\sum_{j}^{n} x_{j} V_{m j} B_{j i}}-\sum_{k}^{n} \frac{x_{k} V_{m i} B_{i k}}{\sum_{j}^{n} x_{j} V_{m j} B_{j k}}-\frac{1}{2}\left[\frac{Z_{i} \sum_{j}^{n} x_{j} B_{j i} \ln B_{j i}}{\sum_{l}^{n} x_{l} B_{l i}}+\sum_{j}^{n} \frac{Z_{j} x_{j} B_{i j}}{\sum_{l}^{n} x_{l} B_{l i}}\left(\ln B_{i j}-\right.\right. \\
& \left.\left.\frac{\sum_{t}^{n} x_{t} B_{t j} \ln B_{t j}}{\sum_{l}^{n} x_{l} B_{l i}}\right)\right]
\end{aligned}
$$

where $V_{m i}$ and $V_{m j}$ are the molar volumes of $i$ and $j$ components in their pure state, respectively, and were attained from the temperature-dependent interactions proposed in [170]. Table 3-3 lists the predicted partial molar volumes, enthalpies, and $\partial \mu_{i} / \partial x_{i}$ for selected liquid molten metal alloys. For the systems containing alkali metal alloys or mercury, the essential parameters in the 
above equations were not available. Therefore, the activity of $\mathrm{Na}$ in liquid Na-K alloy was attained by using the work of Shpilrain et al. [171].

Table 3 - 3: Estimated partial molar volume $\bar{V}_{i}$, enthalpies $\bar{H}_{i}$, and activity parameter $\partial \mu_{1} / \partial x_{1}$ of component at given mole fraction of the first component $\left(x_{i}\right)$ and temperature for selected composition.

\begin{tabular}{lccccccc}
\hline Mixture & $T_{\text {ave }}\left({ }^{\circ} \mathrm{C}\right)$ & $x_{1}$ & $\bar{V}_{1}\left(\mathrm{~cm}^{3} / \mathrm{mole}\right)$ & $\bar{V}_{2}\left(\mathrm{~cm}^{3} / \mathrm{mole}\right)$ & $\bar{H}_{1}(\mathrm{~J} / \mathrm{mole})$ & $\bar{H}_{1}(\mathrm{~J} / \mathrm{mole})$ & $\begin{array}{c}\partial \mu_{1} / \partial x_{1} \\
(\mathrm{~J} / \mathrm{mole})\end{array}$ \\
\hline Sn-Bi & 295 & 0.50 & 21.5 & 19.5 & 99.02 & 99.01 & 11433 \\
\hline $\mathrm{Sn}-\mathrm{Cd}$ & 295 & 0.50 & 13.3 & 17.5 & 1168.30 & 1658.40 & 8068 \\
\hline $\mathrm{Sn}-\mathrm{Zn}$ & 375 & 0.50 & 10.1 & 18.1 & 1487.60 & 3831.30 & 10949 \\
\hline $\mathrm{Sn}-\mathrm{Pb}$ & 295 & 0.50 & 21.0 & 19.0 & 772.47 & 851.89 & 4944 \\
\hline $\mathrm{Sn}-\mathrm{Pb}$ & 450 & 0.50 & 23.4 & 21.4 & 983.27 & 1084.40 & 6314 \\
\hline $\mathrm{Sn}-\mathrm{Ga}$ & 295 & 0.50 & 12.2 & 17.0 & 622.88 & 719.49 & 8847 \\
\hline $\mathrm{Bi}-\mathrm{Pb}$ & 295 & 0.50 & 23.3 & 24.3 & -718.26 & -749.26 & 17460 \\
\hline $\mathrm{Bi}-\mathrm{Sn}$ & 500 & 0.023 & 28.1 & 26.4 & 514.53 & 0.2852 & 46013 \\
\hline
\end{tabular}

\subsection{Model Validation}

Mathematical models are considered validated if their prediction agrees with reliable experimental data. There are many issues that influence the accuracy of experimental data and in turn the validity of numerical models. Gravity is one of the parameters that may affect the accuracy of thermodiffusion data. Gravity can trigger convective flows, particularly in mixtures such as molten metal alloys.

Thermodiffusion coefficient factors in selected mixtures estimated by our proposed equation are shown in Table 3-5. The experimental values and predictions of Eslamian et al. [17] are given in Table 3-5 as well. Our proposed formula, Eq. 3.21 predicted the experimental data more accurately than that of Eslamian et al. [17]. The relative error between the experimental data and the calculated values for the thermodiffusion factor were calculated from a theoretical model expression defined as follows: 
Error $=\frac{\alpha_{\text {Theory }}-\alpha_{\text {Exp. }}}{\alpha_{\text {Exp. }}} \times 100$

Both Eq. 3.21 and the equation of Eslamian et al. fail to predict the sign of the thermodiffusion factor in the Sn-Ga mixture. Nevertheless, Eq. 3.21 shows an overall better prediction power. Note that the Maier's expression, Eq. 3.20, does not account for the electronic forces, and it is therefore incomplete when used for metal-containing mixtures. In addition, Figure 3-1 depicts the predictive trend of variation of the thermodiffusion factor versus mole fraction for various molten metal mixtures at given temperature obtained by the proposed model and also by the Haase [27], Kempers [159], Shukla and Firoozabadi [160], Winter and Drickamer [28], and Eslamian et al. [17] models. It must be noted that for each mixture there is only one experimental data point for a given concentration.

Figure 3-2 shows the thermodiffusion coefficient of the first component of $\mathrm{Sn}-\mathrm{Bi}$, $\mathrm{Sn}-\mathrm{Pb}$, $\mathrm{Bi}-\mathrm{Pb}$, Sn-Ga, and Na-K mixtures versus temperature. To calculate the thermodiffusion coefficient from the thermodiffusion factor, the molecular diffusion coefficient $D_{m}$ should be obtained a priori. As an approximation, the Einstein-Stokes relation was employed to estimate the molecular diffusion coefficient of spherical molecules in a liquid of given viscosity [17].

$D_{m}=\frac{K_{B} T}{6 \pi \eta r}$ 
where $r$ is the hydrodynamic radius of molecules, which are assumed to be spherical. In this work, for the purpose of comparison, it has added this effect to the Maier model and compared the results with the experimental data. The results are listed in Table 3-6.

\subsection{Result and Discussion}

In this research work, it was proposed a model to predict the thermal diffusion factor of a series of binary molten metal mixtures. The model was developed based on the thermal diffusion coefficients of each constituent of a binary molten metal mixture, which were obtained by utilizing the Einstein-Stokes relation. The proposed model is based on the assumption that there are three forces acting on ions [29]. These forces are due to a thermal gradient, an induced concentration gradient, and an induced electric field. Further, it was assumed that these forces are balanced in the steady state.

The proposed model is within the framework of linear non-equilibrium thermodynamics and considers the thermodiffusion factor in terms of the net heats of transport of the electronic and ion-ion thermal interactions. Table 3-4 showed that the proposed model predicts experimentally measured thermal diffusion factors more accurately than do other prominent models. Note that, the data provided on table 3-4 has large deviation as compared to experimental data and it is due to complexity of the material behaviour in molten state. Thus, some values have been estimated and its reflection s the large deviation that is tabulated in table 3-4Table 3-5 shows the results of Maier's model with and without the electron-ion force. As can be seen, the electron-ion force negatively affects the accuracy of Maier's model. Figure 3-1 also reiterates this result visually and shows the comparison of the proposed model to experimental and other numerical models. Figure 3-2 displays the results of the calculation of the 
thermodiffusion coefficients of different binary metal mixtures vs. temperature. The thermodiffusion coefficients vary due to different melting temperatures, variations in molecular dynamics, differences in atomic radii, and varying dynamic viscosities.

Table 3 - 4: Comparison of calculated thermodiffusion factor against the experimental data.

\begin{tabular}{llllllllll}
\hline Mix & $\mathbf{T}_{\text {ave }}(\mathbf{K})$ & $\mathbf{x}_{\mathbf{i}}$ & Exp. & Haase & Kempers & Firoozabadi & Drickamer & Eslamian & $\begin{array}{l}\text { Proposed } \\
\text { Model }\end{array}$ \\
\hline $\mathrm{Sn}-\mathrm{Bi}$ & 568 & 0.5 & 1 & -0.01 & 0.02 & 0 & -5.18 & -3.96 & 1.41 \\
\hline $\mathrm{Sn}-\mathrm{Cd}$ & 568 & 0.5 & -3.5 & 1.41 & 0.26 & -0.06 & 1.4 & -2.63 & -3.98 \\
\hline $\mathrm{Sn}-\mathrm{Zn}$ & 648 & 0.5 & -41 & 7.09 & 1.52 & -0.38 & -19.18 & -30.85 & -40.99 \\
\hline $\mathrm{Sn}-\mathrm{Pb}$ & 568 & 0.5 & -19 & -1.46 & 0.65 & -0.16 & -13.13 & -11.43 & -17.53 \\
\hline $\mathrm{Sn}-\mathrm{Pb}$ & 723 & 0.5 & -8.3 & -1.46 & 0.61 & -0.15 & -7.78 & -6.85 & -9.46 \\
\hline $\mathrm{Sn}-\mathrm{Ga}$ & 568 & 0.5 & -1.8 & 1.01 & -0.28 & 0.07 & 7.89 & 3.74 & 0.99 \\
\hline $\mathrm{Bi}-\mathrm{Pb}$ & 568 & 0.5 & -11.3 & -0.04 & 0 & 0 & 0.05 & -0.72 & -2.6 \\
\hline
\end{tabular}

Table 3 - 5: Experimental and calculated using the Maier model (from Eq. 3.20) and modified model with Electron-Ion forces against the experimental data.

\begin{tabular}{cccccc}
\hline Alloy & $\mathbf{C}_{\mathbf{1}}$ (wt \%) & $\mathbf{T}_{\text {Mean }}(\mathbf{K})$ & $\boldsymbol{\alpha}_{\boldsymbol{T} \text { (exp) }}$ & $\boldsymbol{\alpha}_{\boldsymbol{T} \text { (calculated) }}$ & $\begin{array}{c}\boldsymbol{\alpha}_{\boldsymbol{T} \text { (modified) } \mathbf{w i t h}} \\
\text { electron-electron } \\
\text { forces }\end{array}$ \\
\hline $\mathrm{Bi}-\mathrm{Sn}$ & 4.0 & 773 & $0.758 \pm 0.066$ & $0.785(3.6)$ & 0.8548 \\
\hline $\mathrm{Ag}-\mathrm{Sn}$ & 0.04 & 773 & $-0.175 \pm 0.049$ & $-0.162(-7.4)$ & -0.191 \\
\hline $\mathrm{Co}-\mathrm{Sn}$ & 0.04 & 773 & $-1.86 \pm 0.040$ & $-1.712(-7.9)$ & -1.686 \\
\hline $\mathrm{Au}-\mathrm{Sn}$ & 0.07 & 823 & $0.837 \pm 0.012$ & $0.934(11.6)$ & 0.705 \\
\hline
\end{tabular}




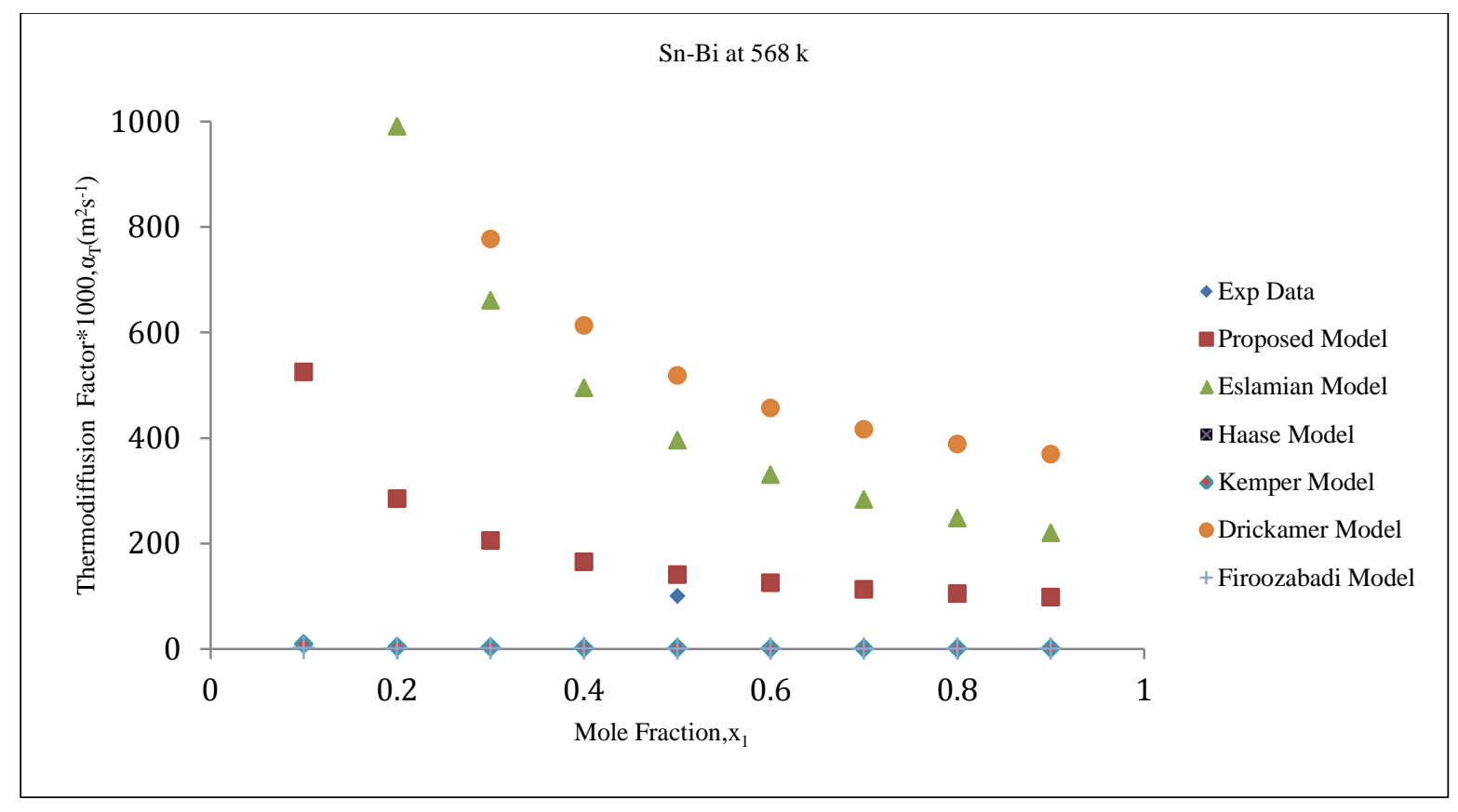

(a)

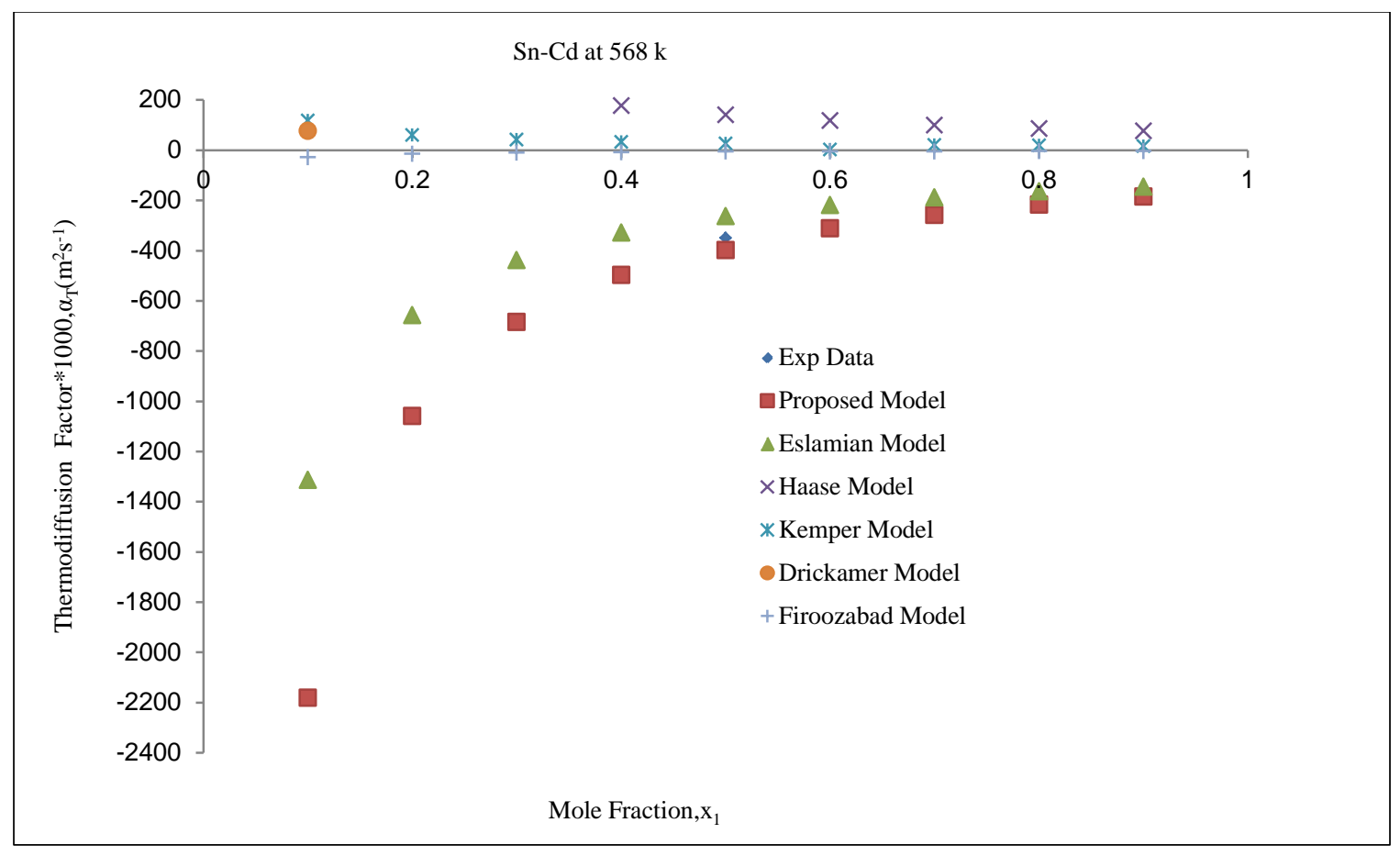

(b) 


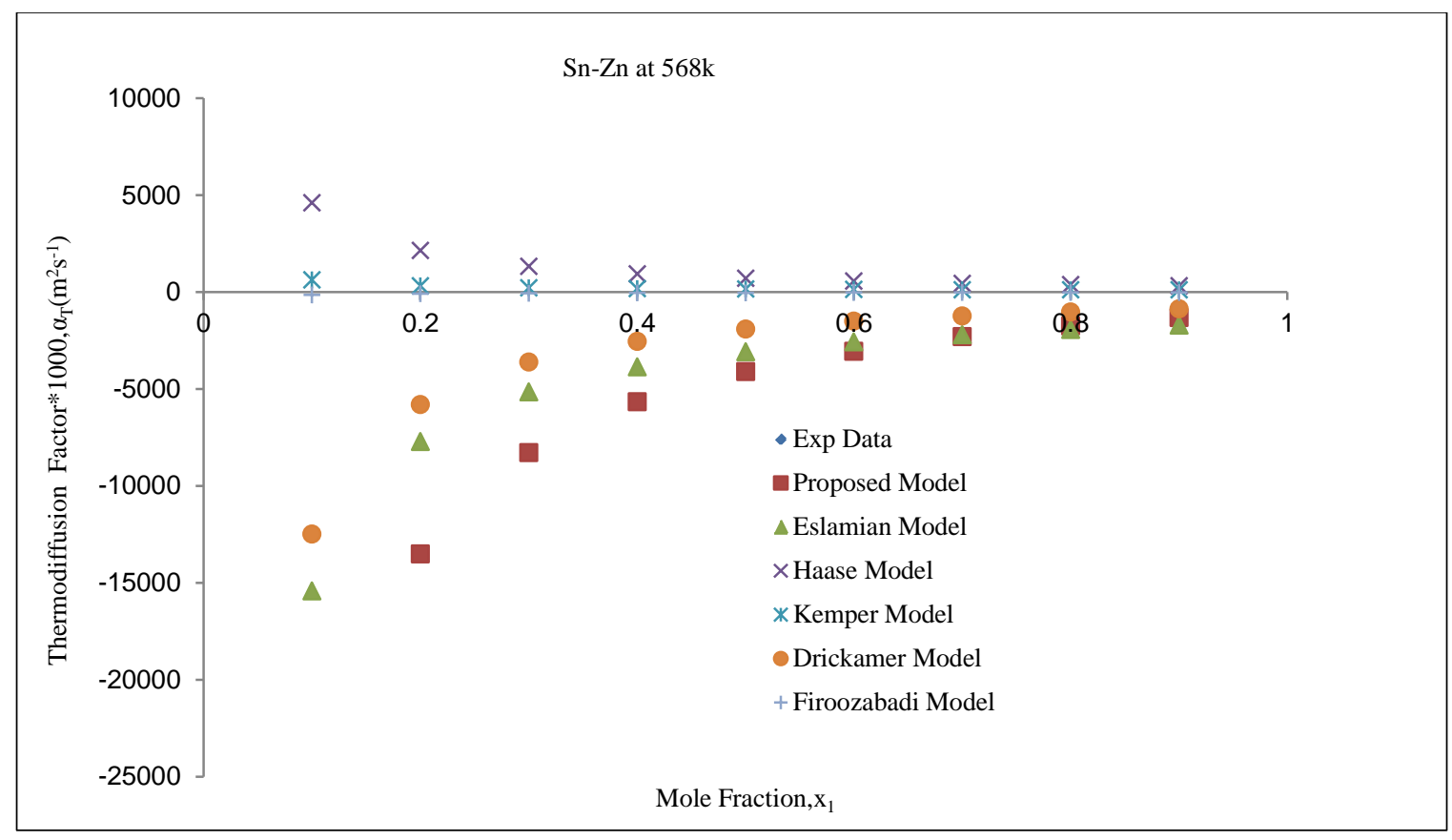

(c)

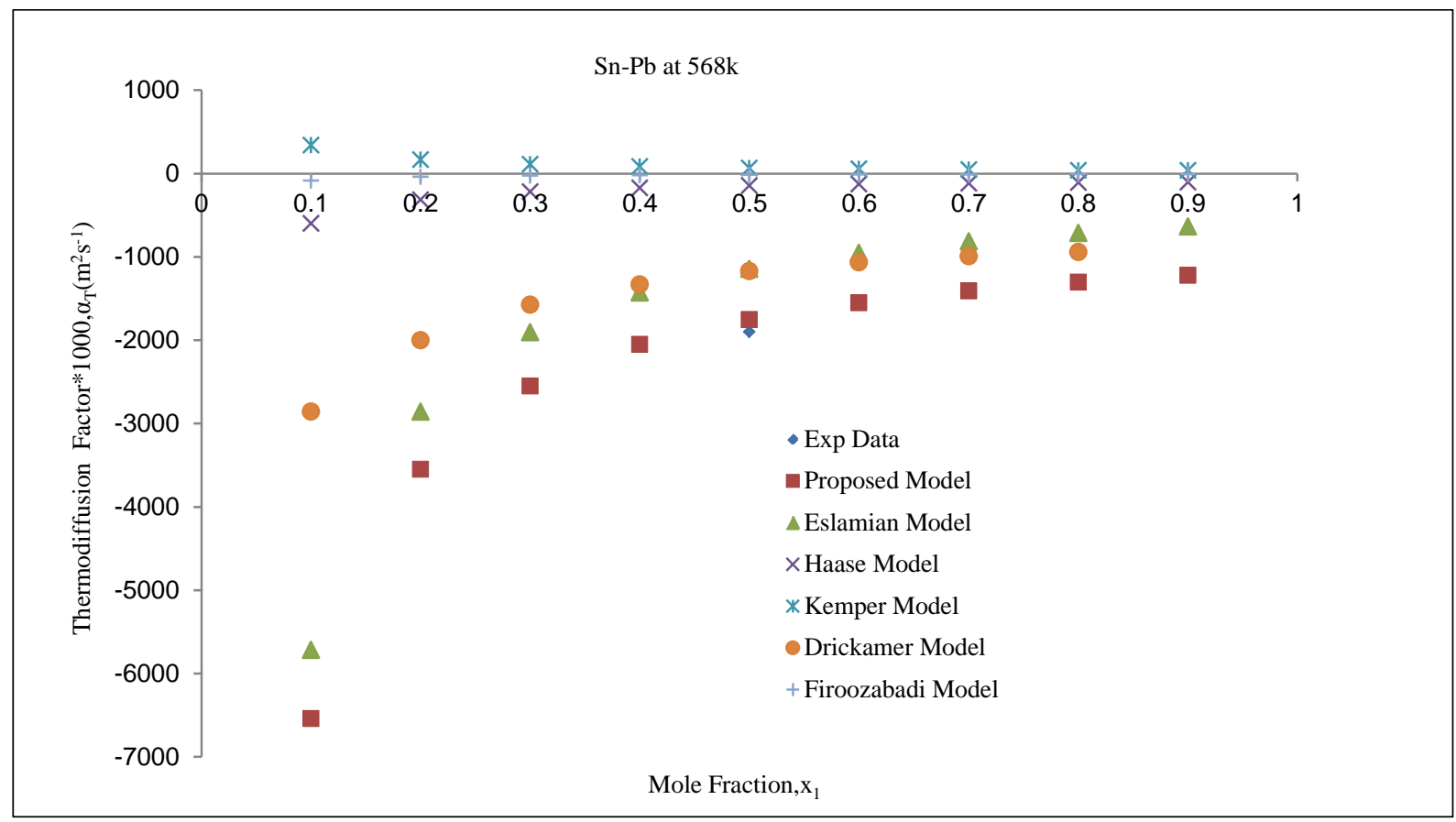

(d) 


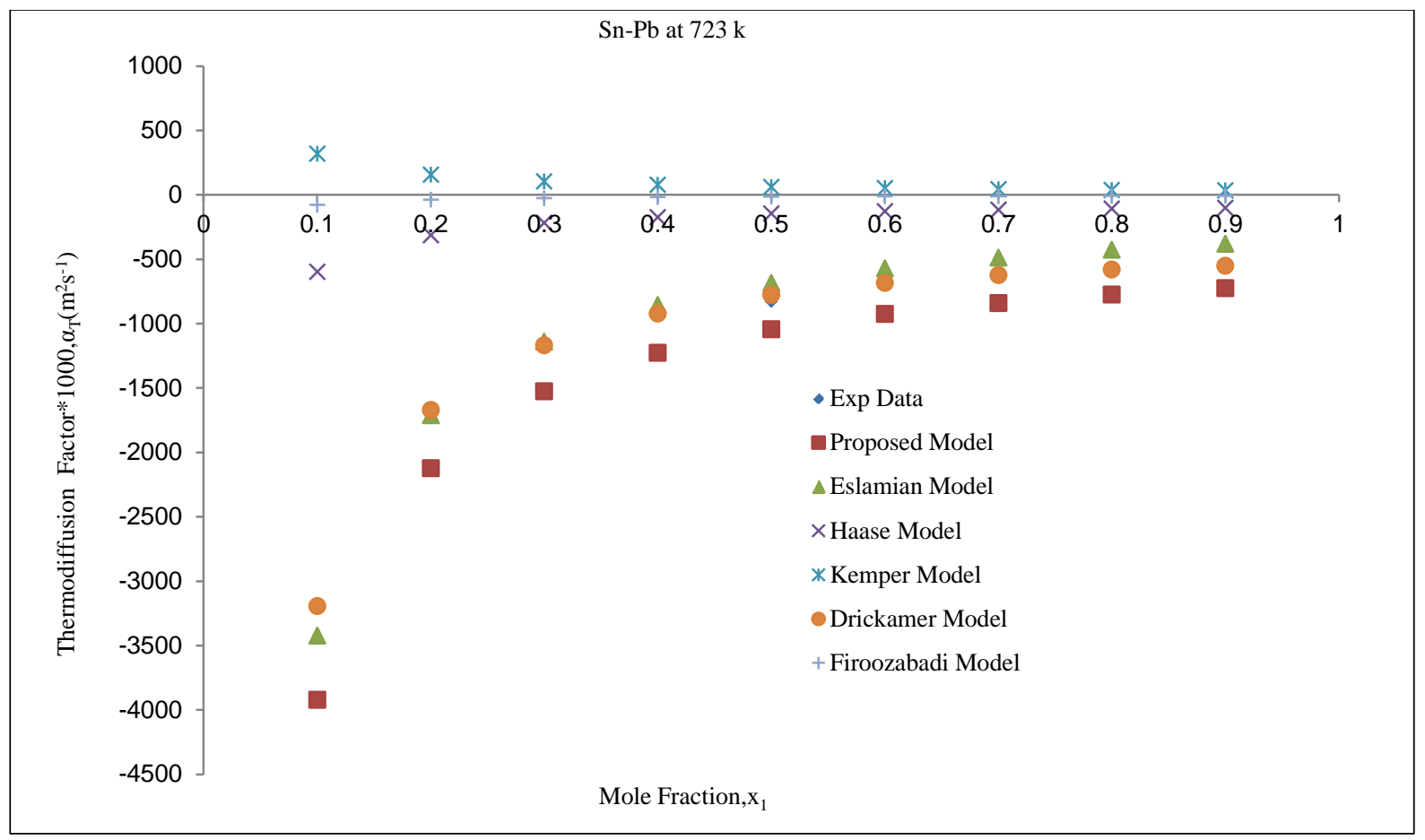

(e)

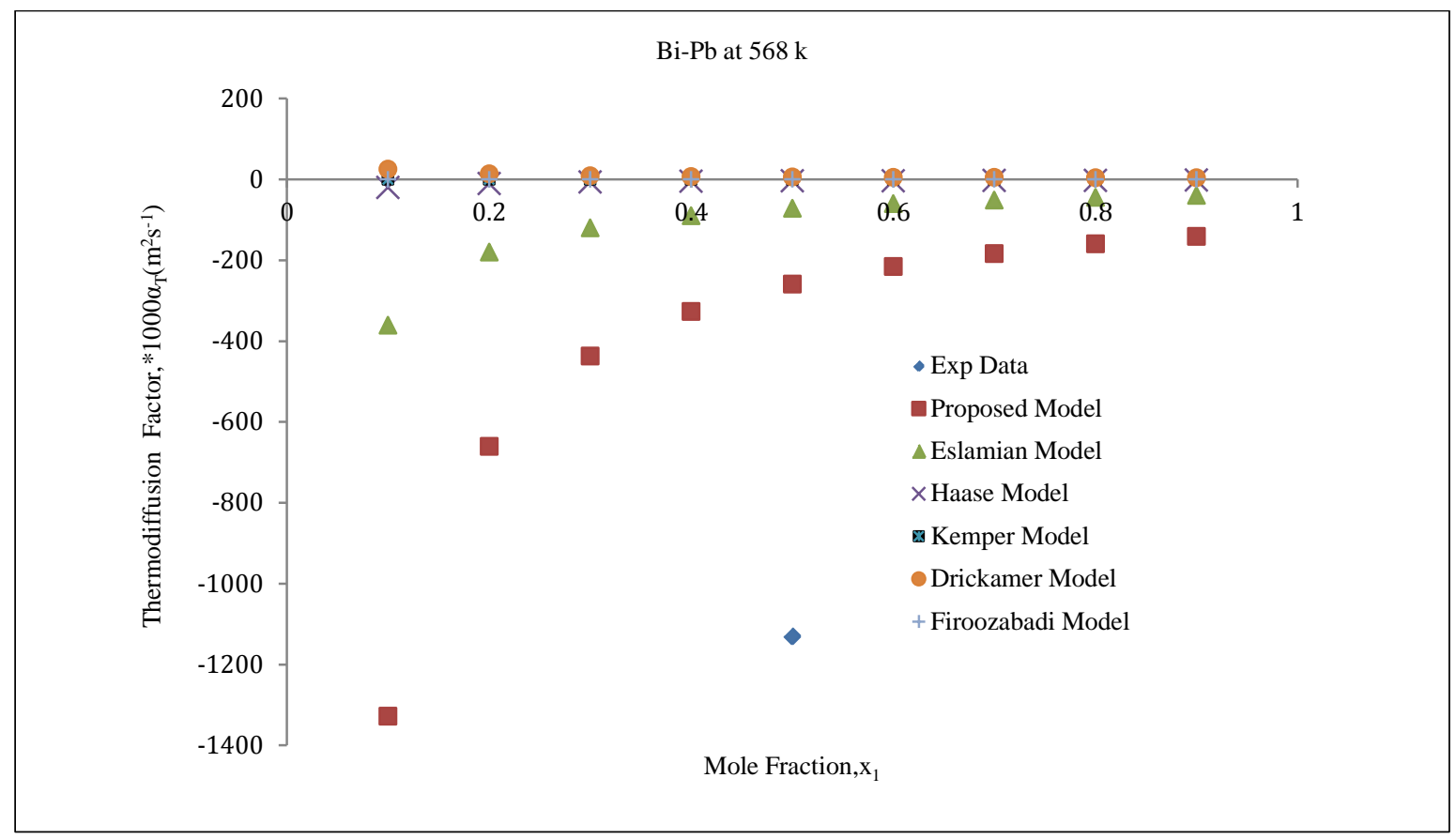

(f) 


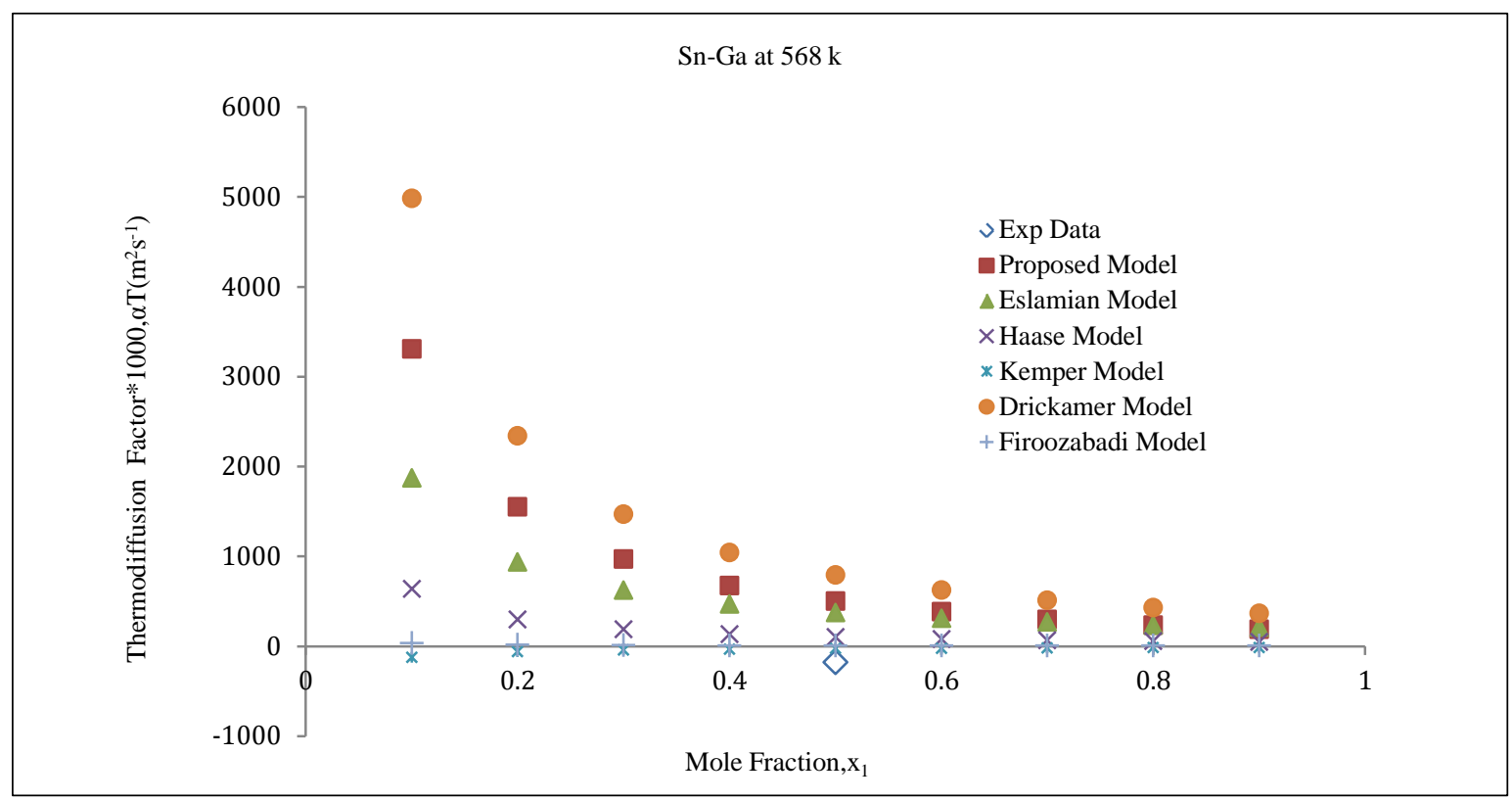

(g)

Figure 3- 1: Effect of concentration on thermodiffusion factors: Comparison of the prediction of different models. Note that the experimental data is only available for an equimolar mixture. (a) $\mathrm{Sn}-\mathrm{Bi}$ at $568 \mathrm{~K}$, (b) Sn-Cd at 568 K, (c) Sn-Zn at 568 K, (d) Sn-Pb at 568 K, (e) Sn-Pb at 723 K, (f) Bi-Pb at 568 K and (g) Sn-Ga at $568 \mathrm{~K}$. 


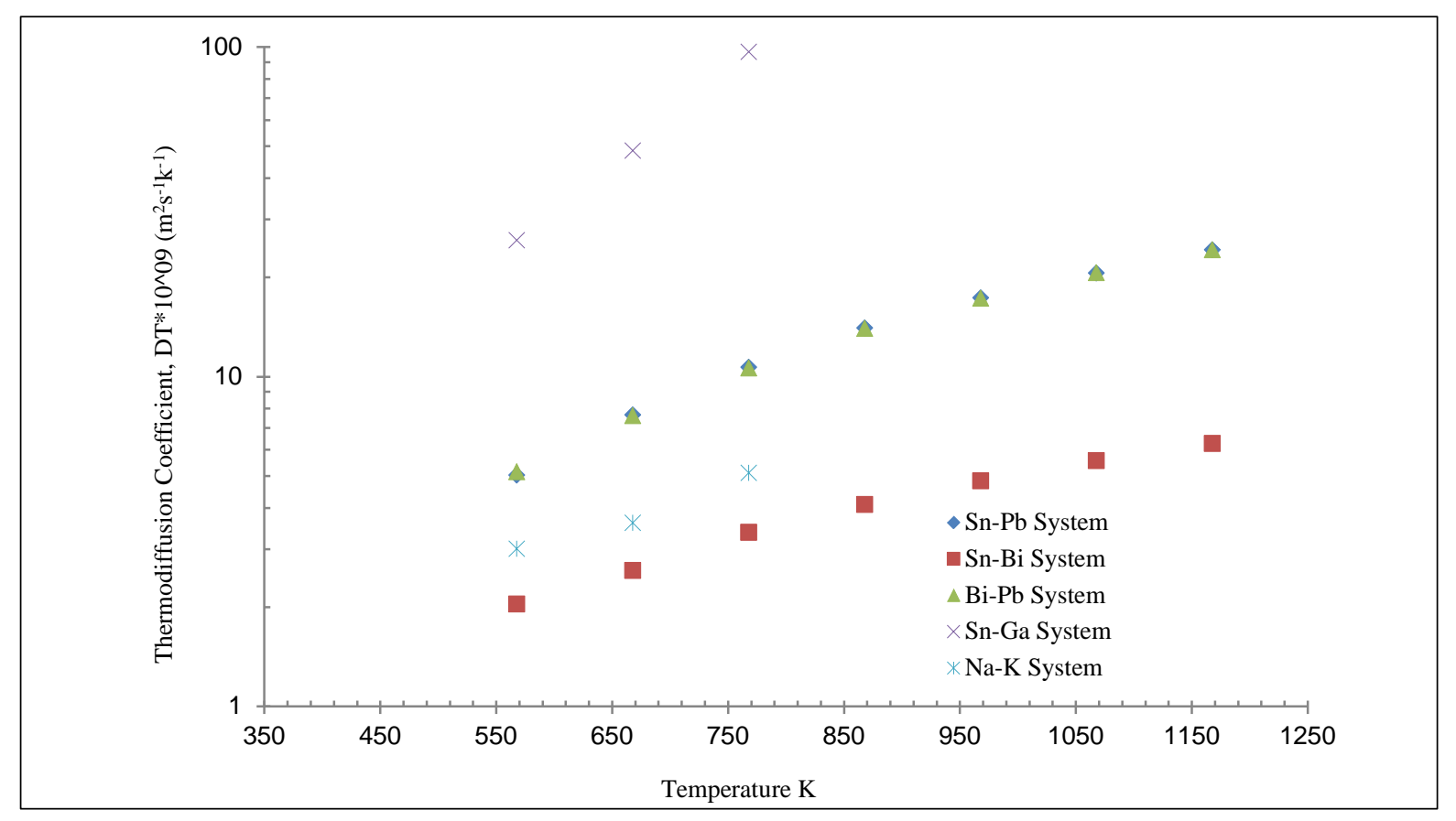

Figure 3- 2: Thermodiffusion coefficient of binary molten metals at various temperatures and $x_{1}=0.5$ by using Stokes-Einstein equation for each component and the proposed model for: $\mathrm{Sn}-\mathrm{Pb}, \mathrm{Sn}-\mathrm{Bi}, \mathrm{Bi}-\mathrm{Pb}, \mathrm{Sn}-$ Ga, and Na-k.

\subsection{Summary}

In this chapter, various mathematical models for the determination of thermodiffusion factor were presented. A detailed explanation of the proposed mathematical expression was also given. The proposed model was derived based on the models of Eslamian [37] and Maier [138]. Furthermore, a comparison of the proposed model with experimental data and with models from other studies was conducted. It was demonstrated that the proposed model resulted in a more accurate prediction of thermodiffusion factors than other models when compared to experimental data, as reflected by Table 3-4. 


\section{CHAPTER FOUR}

\section{The Role of Convection and Radiation Coupled with Thermodiffusion Effect in Binary Molten Metals}

\subsection{Introduction}

This chapter presents the finite element (FE) simulations and the results of the modeling of a binary molten metal in a vertical rectangular cell encapsulated by a quartz container. The effect of external natural convection and surface radiation to the ambient is studied and compared to the case without convection and radiation for both situations of heating from the bottom and top of the cell. This chapter also presents the results of the study of thermosolutal convection in an enclosure filled with a molten Sn-Bi alloy, which was solved by using the transport equations modified to incorporate the thermodiffusion factor, as explained in chapter 3.

In general, the thermal boundary conditions were supposed to be either steady and isothermal or at a constant heat flux. In the present work, it was assumed steady boundary conditions on the top and bottom walls and unsteady boundary conditions on the sidewalls. On the sidewalls, the ambient temperature was assumed constant, and the presence of radiation and natural convection from the outer walls resulted in the unsteady boundary condition.

\subsection{Solution Technique}

Significant effort has been focused toward the improvement of approximate solutions by applying numerical methods. One of these numerical methods, which was first developed for 
stress analysis, is the finite element method. These days, finite element technique is commonly used in fluid mechanics, heat transfer, and many other areas. Finite element method is a powerful technique for finding the numerical solution to differential and integral equations that occur in several fields of engineering and applied science.

The basis of finite element technique comes from the classical variation method and the weighted residual method. The finite element technique behaves as a geometrically complex domain with an assembly of simple geometric sub-domains, known as finite elements. Therefore, the estimated function for each small element is developed so that any continuous function can be signified by a linear arrangement of algebraic polynomials. The interpolation functions are described in terms of the values of the field variable at particular points, known as nodes, on the boundary or inside the element, that absolutely explain the performance of the field variable within the element. When the nodal values are determined, the interpolation functions describe the field variable within the assembly of the elements.

Finite element technique was used to assess and investigate the concentration, velocity, and temperature distributions within the quartz enclosure. For convergence checking between two successive time steps (iteration), the next equations need to be satisfied: $\frac{\left\|\Delta u_{i}\right\|}{\|u\|} \leq \varepsilon_{u}, \Delta u_{i}=$ $u_{i}-u_{i-1}$, and $\frac{\left\|R_{i}\right\|}{\left\|R_{0}\right\|} \leq \varepsilon_{F}$ are applied where $u_{i}$ represents the temperature, velocities, and concentration along the $\mathrm{z}$ and $r$ axes for each node and $R_{0}$ is a reference vector. $R_{0}$ is generally used as $R\left(u_{i}\right)$, which is the residual force vector. $\varepsilon_{u}$ is the convergence tolerance according to the relative error and $\varepsilon_{F}$ is the residual convergence tolerance for each variable at each iteration. Subsequently, both $\Delta u_{i}$ and $R\left(u_{i}\right)$ tend to be zero near the solution, so the problem is presumed converged when the system satisfies the above condition within a factor of $10^{-4}$ for both the solution vector and the residual force vector. For our work, the maximum number of nonlinear 
iterations for fluid problem was fixed to 450 . The norm $\|||$ is a root mean square norm summed to total the equations for the model [172].

To explain convective heat transfer problems with a mathematical formulation, the complete nonlinear Navier-Stokes equations for laminar, incompressible Newtonian flows in the steady-state condition together with the energy and mass equations were solved numerically using the finite element (FE) technique [172].

\subsection{Governing Equations and Boundary Conditions}

The continuity, concentration, momentum, and energy balance equations were solved in order to study the flow, concentration, and heat transfer characteristics of the problem. The governing equations were rendered dimensionless by using the following non-dimensional variables [30]:

$U=\frac{u}{u_{0}} \quad V=\frac{v}{v_{0}} \quad X=\frac{x}{L} \quad Y=\frac{y}{L} \quad P=\frac{P L}{\eta u_{0}} \quad \theta=\frac{T-T_{c}}{\Delta T} \quad C=\frac{c-c_{0}}{\Delta c} \quad \Delta T=T_{H}-T_{C}$

$u_{0}=\sqrt{g \beta_{T} \Delta T L}$

where $U$ and $V$ are dimensionless velocities, $P$ is the dimensionless hydrodynamic pressure, $\theta$ is non-dimensional temperature, $C$ is the non-dimensional concentration of a mixture component, $g$ is the gravitational acceleration, $u_{0}$ is a reference velocity, $\Delta T$ is the initial temperature difference, and $L$ is a characteristic length.

Dimensional analysis is another useful tool of modern fluid mechanics. It is the mathematics of dimensions of quantities [173]. This method was used to reduce the number and 
complexity of experimental variables, which affect a given physical phenomenon, by using a kind of compacting technique. It was used to make all of the relevant governing equations, such as Navier-Stokes, to become non-dimensional, a property that further enhances the speed of the computation processes. All of the non-dimensionalization processes are explained step-by-step in Appendix-A.

\section{Continuity equation}

$$
\begin{aligned}
& \frac{\partial u}{\partial x}+\frac{\partial v}{\partial y}=0 \\
& \frac{\partial U}{\partial X}+\frac{\partial V}{\partial Y}=0
\end{aligned}
$$

(Non-Dimensional)

\section{Concentration equation}

$$
\begin{aligned}
& \rho_{f}\left[u \frac{\partial C}{\partial x}+v \frac{\partial C}{\partial y}\right]=\rho_{f} \cdot D_{m}\left[\frac{\partial^{2} C}{\partial x^{2}}+\frac{\partial^{2} C}{\partial y^{2}}\right]-\rho_{f} \cdot D_{T}\left[\frac{\partial^{2} T}{\partial x^{2}}+\frac{\partial^{2} T}{\partial y^{2}}\right] \quad \text { (Dimensional) } \\
& R e\left[U \frac{\partial C}{\partial X}+V \frac{\partial C}{\partial Y}\right]=\frac{1}{S c}\left\{\left[\frac{\partial^{2} C}{\partial X^{2}}+\frac{\partial^{2} C}{\partial Y^{2}}\right]+\alpha_{T}\left[\frac{\partial^{2} \theta}{\partial X^{2}}+\frac{\partial^{2} \theta}{\partial Y^{2}}\right]\right\}_{\text {(Non-Dimensional) }}^{\text {(Non) }}
\end{aligned}
$$

\section{Momentum equation}

$X$-direction

$$
\begin{aligned}
& {\left[u \frac{\partial u}{\partial x}+v \frac{\partial u}{\partial y}\right]=-\frac{\partial p}{\partial x}+\mu\left[\frac{\partial^{2} u}{\partial x^{2}}+\frac{\partial^{2} u}{\partial y^{2}}\right]} \\
& R e\left[U \frac{\partial U}{\partial X}+V \frac{\partial U}{\partial Y}\right]=\frac{\partial P}{\partial X}+\left[\frac{\partial^{2} U}{\partial X^{2}}+\frac{\partial^{2} U}{\partial Y^{2}}\right]
\end{aligned}
$$

(Dimensional)

$$
\text { ( Non-Dimensional) }
$$

Y-direction

$\rho_{f}\left[u \frac{\partial v}{\partial x}+v \frac{\partial v}{\partial y}\right]=-\frac{\partial p}{\partial y}+\mu\left[\frac{\partial^{2} v}{\partial x^{2}}+\frac{\partial^{2} v}{\partial y^{2}}\right]-\rho_{f} \cdot g\left[\beta_{T}\left(T-T_{0}\right)-\beta_{C}\left(C-C_{0}\right)\right]$ 
(Dimensional)

$$
R e\left[U \frac{\partial V}{\partial X}+V \frac{\partial V}{\partial Y}\right]=\frac{\partial P}{\partial X}+\left[\frac{\partial^{2} V}{\partial X^{2}}+\frac{\partial^{2} V}{\partial Y^{2}}\right]-R e[\theta-N C]
$$

(Non-Dimensional)

\section{Energy equation}

$$
(\rho C p)_{f}\left[u \frac{\partial T}{\partial x}+v \frac{\partial T}{\partial y}\right]=k_{f}\left[\frac{\partial^{2} T}{\partial x^{2}}+\frac{\partial^{2} T}{\partial y^{2}}\right]
$$

$$
R e \cdot P r\left[U \frac{\partial \theta}{\partial X}+V \frac{\partial \theta}{\partial Y}\right]=\left[\frac{\partial^{2} \theta}{\partial X^{2}}+\frac{\partial^{2} \theta}{\partial Y^{2}}\right]
$$

(Non-Dimensional)

\section{Surface radiation equation}

$q_{r}=\varepsilon \sigma\left(T_{a m b}^{4}-T^{4}\right)$

(Dimensional)

$$
Q_{r}=\varepsilon_{i}\left[\left(1+\frac{\theta}{\theta_{0}}\right)^{4}-\sum_{j} F_{i j} G_{j}\right]
$$

(Non-Dimensional)

Where, in the above equations, $S_{c}=\mu / \rho D$ is the Schmidt number, $\alpha_{T}=T D_{T} / D$ is the thermodiffusion factor, $\operatorname{Re}=\left(\rho_{o} \cdot u_{o} . L\right) / \mu$ is the Reynolds number, and $\operatorname{Pr}=v / \alpha$ is the Prandtl number. The aim of this work is to study the distribution of temperature and concentration due to the presence of thermodiffusion using the proposed thermodiffusion factor as one of the variables within a cavity filled with a Sn-Bi molten metal binary mixture and in the presence of surface radiation to ambient and external natural convection. Equations (4.2) to (4.7) were solved numerically using the finite element technique to achieve this goal. 


\subsection{Numerical Modeling of Natural Convection}

Two numerical simulations were carried out on the natural convection of a binary molten metal alloy of Sn-Bi mixture enclosed in a cavity incorporating thermodiffusion and heat loss by surface radiation to ambient. The flow was assumed to be laminar and incompressible. In this simulation, a constant heat source was applied to model the temperature difference at the opposing boundaries. Two configurations were studied: top heating and bottom heating, both in a rectangular cavity encapsulated by quartz walls. Heat losses by natural convection and surface radiation from the vertical walls to the ambient were taken into consideration. Material properties for the model are given in Table 4-1.

Table 4 - 1: The physical properties of Sn-Bi mixture at $295^{\circ} \mathrm{C}[25]$ and the references therein.

\begin{tabular}{|c|c|c|}
\hline Physical Properties & Symbols & Sn-Bi \\
\hline Initial Concentration for Sn & $\mathrm{C}_{\mathrm{i}}$ & 0.05 \\
\hline Dynamic Viscosity & $\eta$ & $16 \times 10^{-3}$ g.cm ${ }^{-1} \cdot \mathrm{s}^{-1}$ \\
\hline Density & $\rho$ & 8.325 g.cm ${ }^{-3}$ \\
\hline Hot Temp & $\mathrm{T}_{\mathrm{H}}$ & $307^{\circ} \mathrm{C}$ \\
\hline Cold Temp & $\mathrm{T}_{\mathrm{C}}$ & $283^{\circ} \mathrm{C}$ \\
\hline Conductivity & $\mathrm{K}$ & $0.381 \mathrm{~J}^{-\mathrm{s}^{-1}} \cdot \mathrm{cm}^{-1} \cdot \mathrm{k}^{-1}$ \\
\hline Specific Heat Capacity & $\mathrm{C}_{\mathrm{p}}$ & $0.1745 \mathrm{~J} \cdot \mathrm{g}^{-1} \cdot \mathrm{k}^{-1}$ \\
\hline Prandtl Number & $\mathrm{Pr}$ & $7.33 \times 10^{-3}$ \\
\hline Solutal Expansion & $B c$ & $48.02 \times 10^{-4}$ \\
\hline Thermal volume expansion & $\beta_{T}$ & $1.01 \times 10-{ }^{4}\left(\mathrm{~K}^{-1}\right)$ \\
\hline Kinematic Viscosity & $v$ & $1.922 \times 10^{-} \mathrm{cm}^{2} \cdot \mathrm{s}^{-1}$ \\
\hline Thermal Diffusivity & $\alpha$ & $0.2622 \mathrm{~cm}^{2} . \mathrm{s}^{-1}$ \\
\hline Characteristic Velocity & $u_{o}$ & $0.2622 \mathrm{~cm} . \mathrm{s}^{-1}$ \\
\hline Reynolds Number & $\operatorname{Re}$ & 136 \\
\hline Soret Coefficient & $S_{T}$ & $0.248\left(\mathrm{~K}^{-1}\right)$ \\
\hline Schmidt Number & Sc & 101.566 \\
\hline Thermodiffusion Factor & $\alpha_{T}$ & 0.141 \\
\hline
\end{tabular}




\subsection{Model Geometry and Boundary Conditions}

Figure 4-1 shows the geometry of the rectangular cavity filled with the binary molten metal. The cell is $10 \mathrm{~mm}$ in the vertical direction with two quartz plates of $1 \mathrm{~mm}$ thick, one at the bottom and one on the top. The width of the model cell is $3 \mathrm{~mm}$ in the horizontal direction with two quartz plates of $1 \mathrm{~mm}$ thick on each side. The thermal boundary conditions here dictate heat losses to the surrounding through radiation and external natural convection from the vertical walls. Two cell configurations were considered, one with top heating and one with bottom heating. A constant heat source was applied to the top surface in the first simulation, and the hot and cold surfaces were reversed in the second simulation. Furthermore, the velocity and the mass flux $\left(J_{i}\right)$ at all external walls of the cavity were assumed zero, given that this is a boundarydriven flow problem.

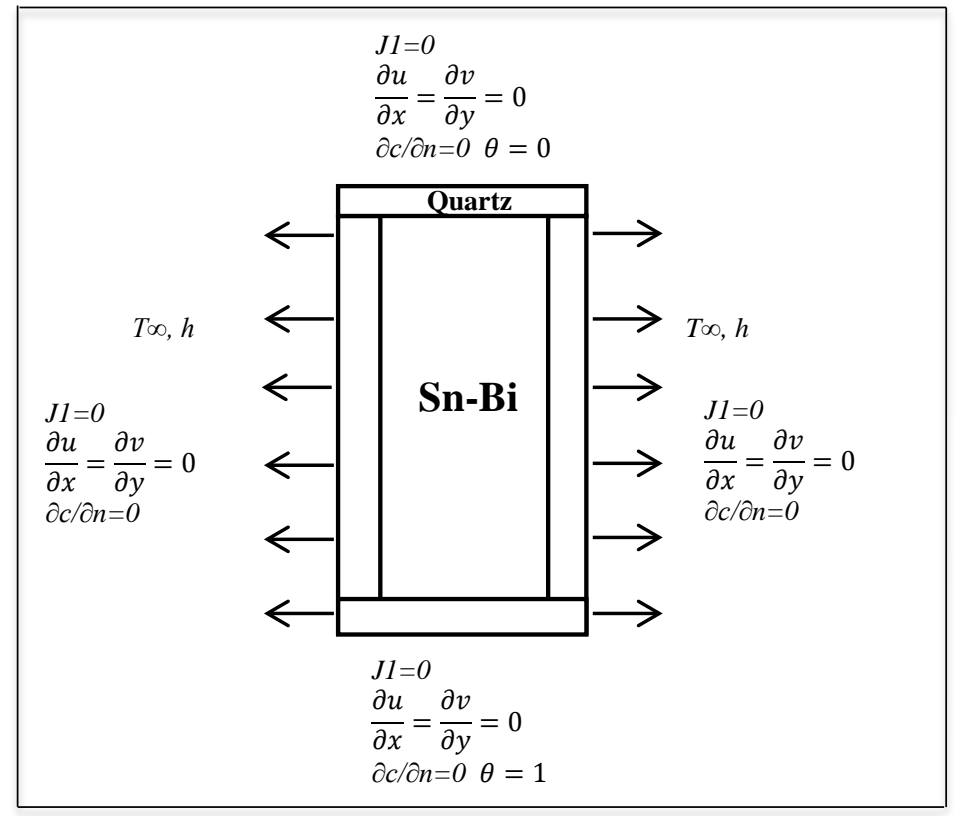

Figure 4 - 1: Computational cell geometry and boundary conditions 


\subsection{Mesh Sensitivity Analysis}

In general, the final results of the numerical simulations can be sensitive to the mesh size. A series of simulations with identical boundary conditions were performed to study the mesh sensitivity and to determine the optimal mesh size. The model originally was constructed with 300 elements and after series of refinements, the model with 4556 elements and 2356 node was rendered insensitive to mesh size. In addition, the mesh at the boundary was further refined to achieve a greater accuracy of temperature and velocity variation at the boundaries. As the first step, a model with a coarse mesh was used to simulate the fluid behaviour. In order to check the mesh independency, the mesh was refined. The process of refining was stopped, as the simulation results from the previous simulation to the current simulation did not change by more than a few percent. This procedure ensured that the numerical results were not sensitive to the mesh size and the results were consistent.

\subsection{Results and Discussion}

The transport equations combined with the Soret effect were solved in a rectangular quartz cell containing a molten Sn-Bi mixture. Dimensionless solutions for the flow with different Raleigh numbers for the top and bottom heating cases with thermodiffusion, surface radiation, and external natural convection effect are shown in Figures 4-2 to 4-5. The main purpose of this study was to investigate the effect of thermodiffusion, surface radiation, and external natural convection on heat transfer in a molten Sn-Bi mixture.

\subsubsection{Case 1: Top Heating Condition}


Figure 4-2 shows temperature and concentration distribution along the rectangular cavity when heating from the top surface. Consequently, the bottom surface was considered to be the cold surface. Simulation results revealed that the system is in a purely diffusive regime. Thermodiffusion effects showed separation of the solute in the molten metal alloy. Heat losses to the environment proved not to have any major effect on the separation process.

Figure 4-2 (a) shows the contour plot of the temperature distribution. In Figure 4-2 (b), the horizontal axis is the distance along the vertical direction and the vertical axis is the temperature distribution. The temperature decreases linearly from the top surface to the bottom surface. As the graph indicates, the temperature gradient is constant along the vertical walls since it is a purely diffusive regime. Figure 4-2 (c) depicts the contour plot of the concentration of Sn and Figure 4-2 (d) depicts the distribution of the Sn concentration. In this simulation, the initial concentration of Sn was set at 0.05 . In the top heating case, thermodiffusion causes the Sn component to migrate toward the cold bottom surface and so the Bi migrates to the top heated plate. Thus, a linear separation occurs. The thermodiffusion factor is positive for Sn, and a positive thermodiffusion factor describes the migration of Sn particles from the hot zone to the cold zone [30]. The pattern is displayed in Figure 4-2 showing the Sn concentration increases by nearly $4 \%$ at the cold zone and decreases by the same amount near the hot zone. However, the concentration changes linearly as a function of the height of the cavity. For temperature, the concentration distribution is linear with a constant gradient [173]. These results for the variation of the temperature in a binary mixture resemble those of a single component fluid in the top heating configuration. Radiation and natural convection also did not affect the temperature and concentration profiles. 
Figure 4-3 shows the effects of natural convection from the outer surfaces of the sidewalls and radiation to the ambient on the temperature and concentration distributions. As these graphs indicate, in the range of temperatures considered here, the effect of natural convection and radiation are negligible. 


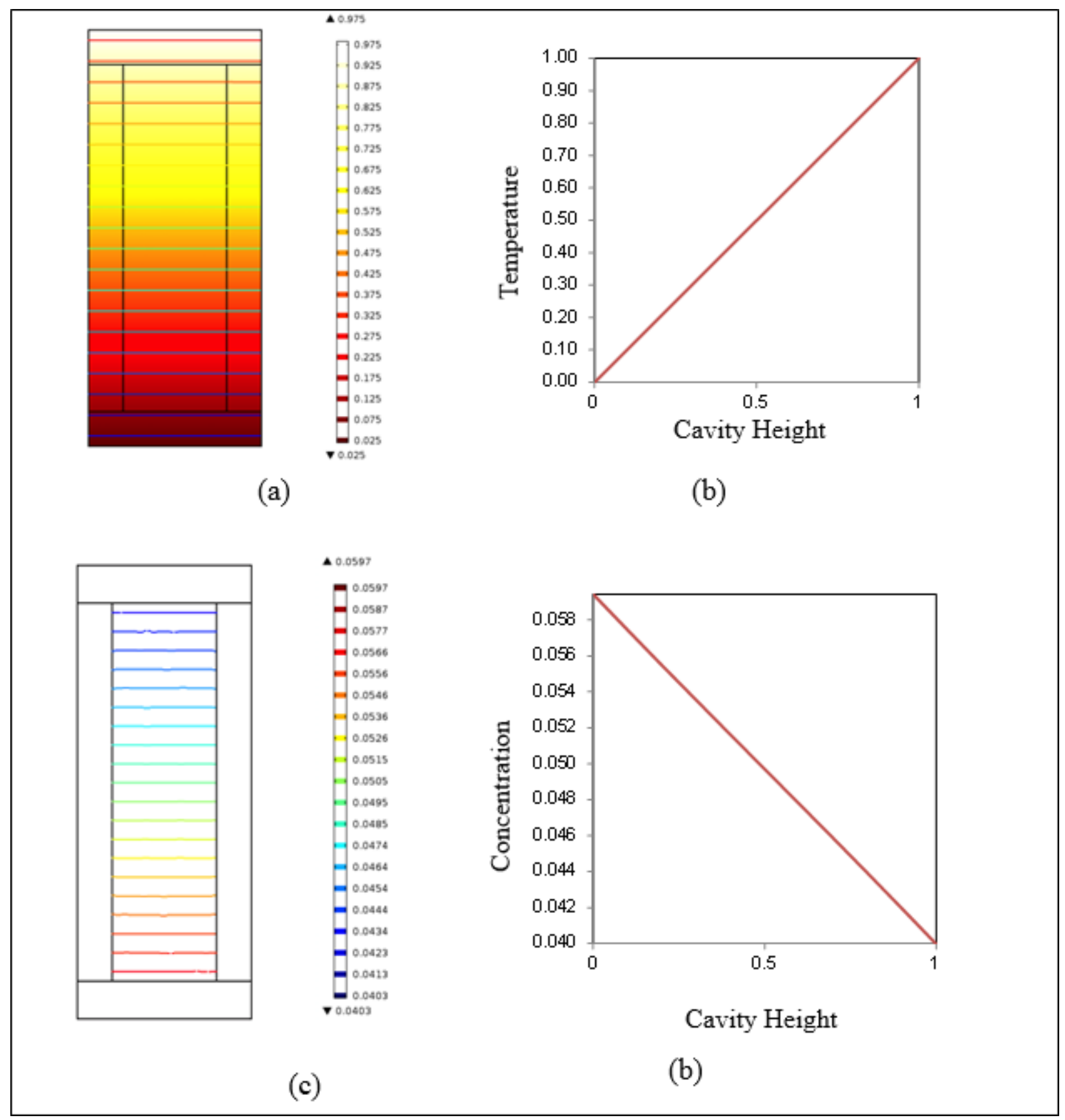

Figure 4 - 2: Temperature and concentration distribution with top heating condition; (a) Lines of constant temperature; (b) Temperature distribution along the cavity height; (c) Lines of constant concentration; (d) Concentration variation along the cavity height. 


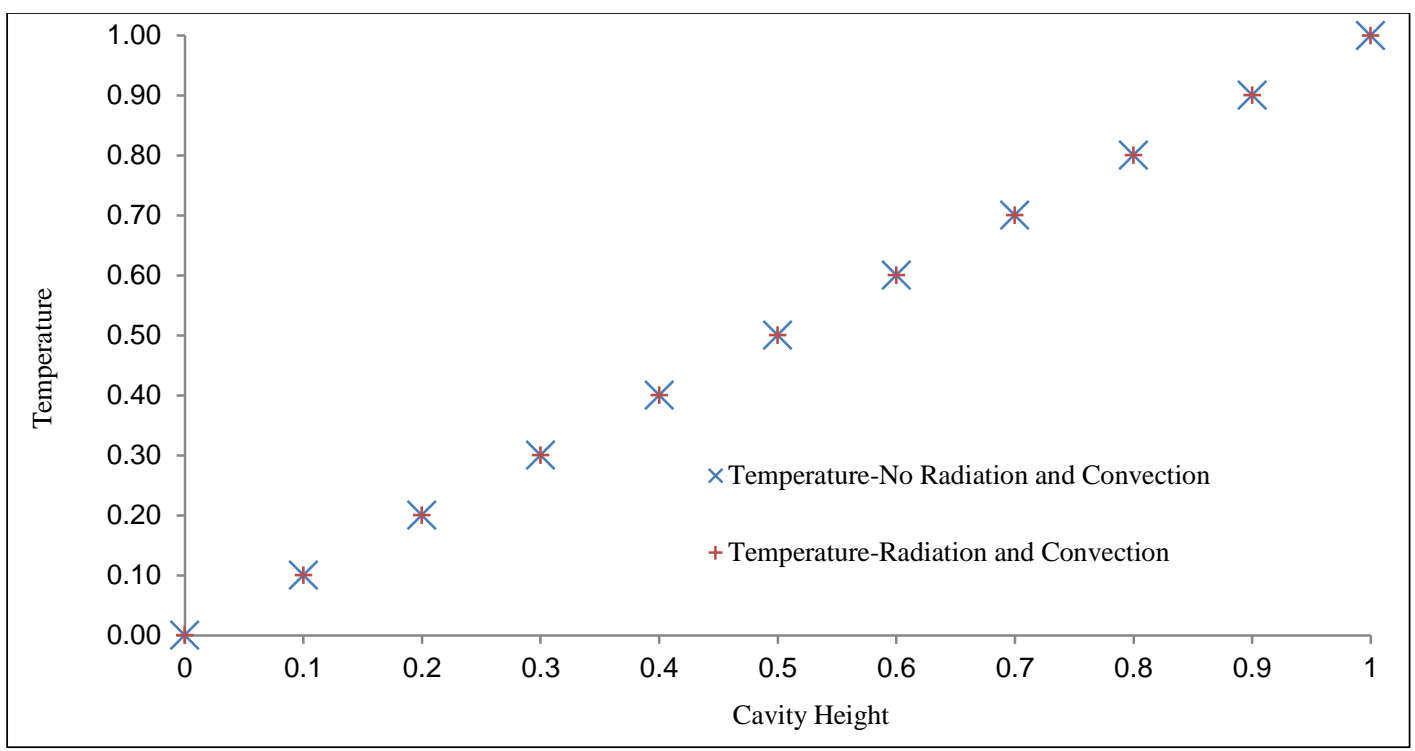

(a)

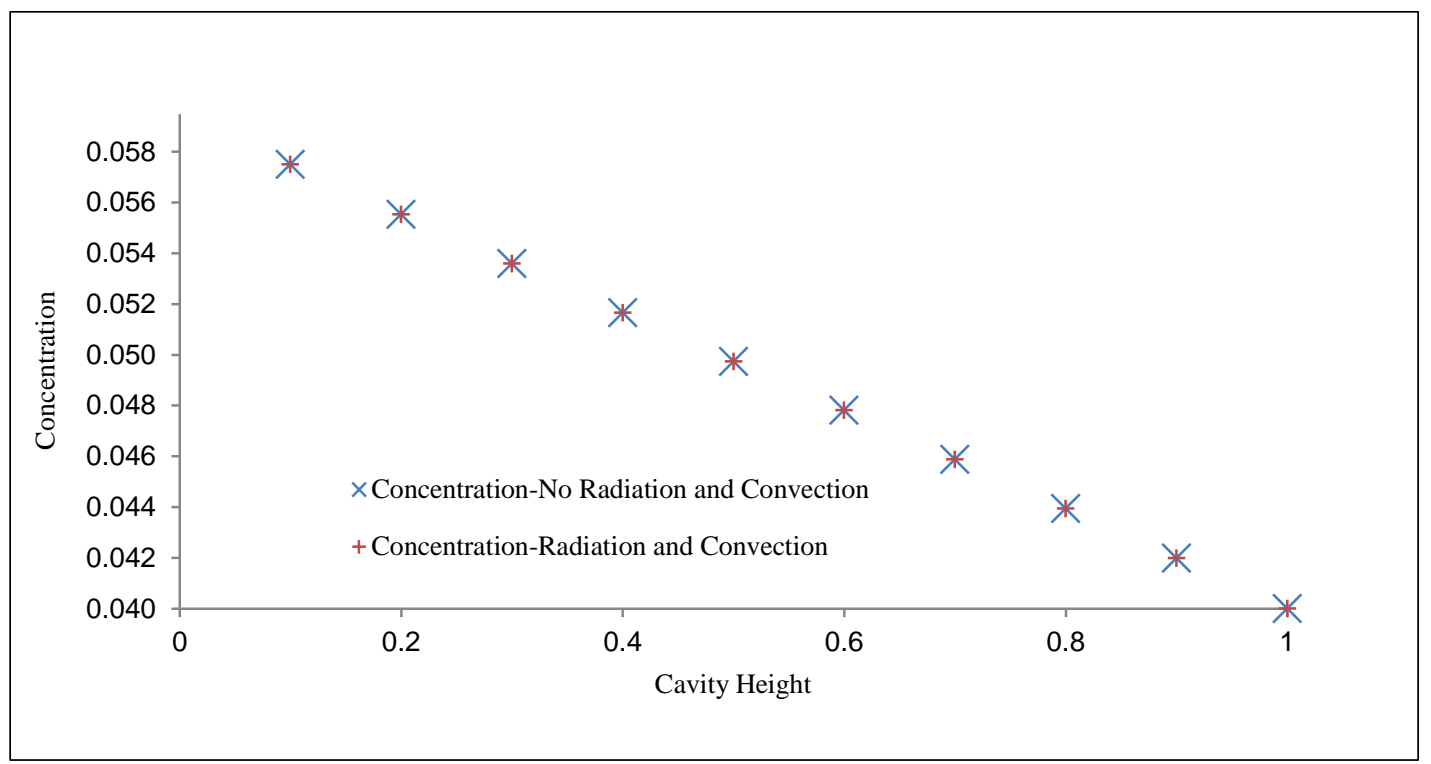

(b)

Figure 4 - 3: Comparison of (a) temperature distribution, and (b) Concentration distribution by applying surface radiation and external natural convection on vertical walls to the ambient in Sn-Bi filled square cavity with thermodiffusion effect at $\mathrm{Ra}=1000$.

\subsubsection{Case 2: Bottom Heating Condition}

In the case of bottom heating (Rayleigh-Benard configuration), it was studied the effects

of the Rayleigh number at $\mathrm{Ra}=1000,2000, \ldots, 10000$ on the flow structure. In this case, since 
the buoyancy force is present, once the flow becomes unstable convective heat transfer becomes one of the major modes of heat and mass transfer in the cell. As the Ra number increases, the destabilizing buoyancy force acting upward increases and outweighs the viscous forces and thermodiffusion and thereby the flow becomes disturbed and unstable. This is due to the fluid particles rising upward from the heated bottom surface as the result of the temperature gradient [30]. The circulatory fluid motion in the cell manifests itself as a convective flow. In turn, this convective flow causes the temperature and concentration gradient to deviate from linearity. Figure 4-4 (a) and Figure 4-4 (b) depict the temperature and concentration distributions, respectively, and Figure 4-5 shows the effect of the Ra number on the concentration distribution.

Figure 4-4 (a) shows the temperature distribution for 10 different Rayleigh numbers. It can be seen that at a Ra number of 1000 , the distribution is almost linear, and as Ra increases (2000 and higher), the nonlinearity becomes more noticeable. The isotherms show that temperature rises from left to right. This is due to the fact the flow is in a counter clockwise direction, and the high temperature fluid molecules move upward closer to the right wall and low temperature fluid molecules move downward closer to the left wall. Here, thermodiffusion, in addition to buoyancy, forces the Sn atoms/ions upward. In contrast, the gravity applies a downward force on the Sn atoms, and the resultant total force generates a counter clockwise flow. As Figure 4-4 (a) indicates, the temperature variation increases with an increase in the Ra number giving rise to a more agitated counter clockwise flow.

Figure 4-4 (b) shows the concentration distribution of Sn as the Ra number increases. It clearly demonstrates that the concentration distribution closely follows the temperature distribution. In this case, Sn atoms migrate from the bottom to the top due to their positive thermodiffusion factor, and the concentration of Sn atoms at the top of the cell increases 
compared to the bottom. However, the non-linearity of the Sn distribution is due to the convective regime, as mixing reduces the separation process. Figure 4-5 graphically shows the concentration distribution. This figure clearly demonstrates that as the Ra number increases, the concentration distribution asymptotically reaches a uniform distribution at the centre of the cavity. From a purely diffusive regime to a convective regime, the concentration distribution of Sn changes from a linear to a nonlinear distribution. The strong mixing at the centre of the cavity creates a constant Sn concentration with a large solute gradient near the hot and cold walls. Such a configuration is not suitable for solidification processes and should be avoided.

For the Reynolds number of 136 that is characteristic of laminar flow, our results showed that the effect of external natural convection and radiation are negligible and the temperature and concentration distributions do not vary significantly compared to the insulated wall conditions. Also, in the case of bottom heating, when the Ra number is large, internal natural convection is dominant, convective flows are strong, and thermodiffusion becomes less important as the mixing of the species prevails. 


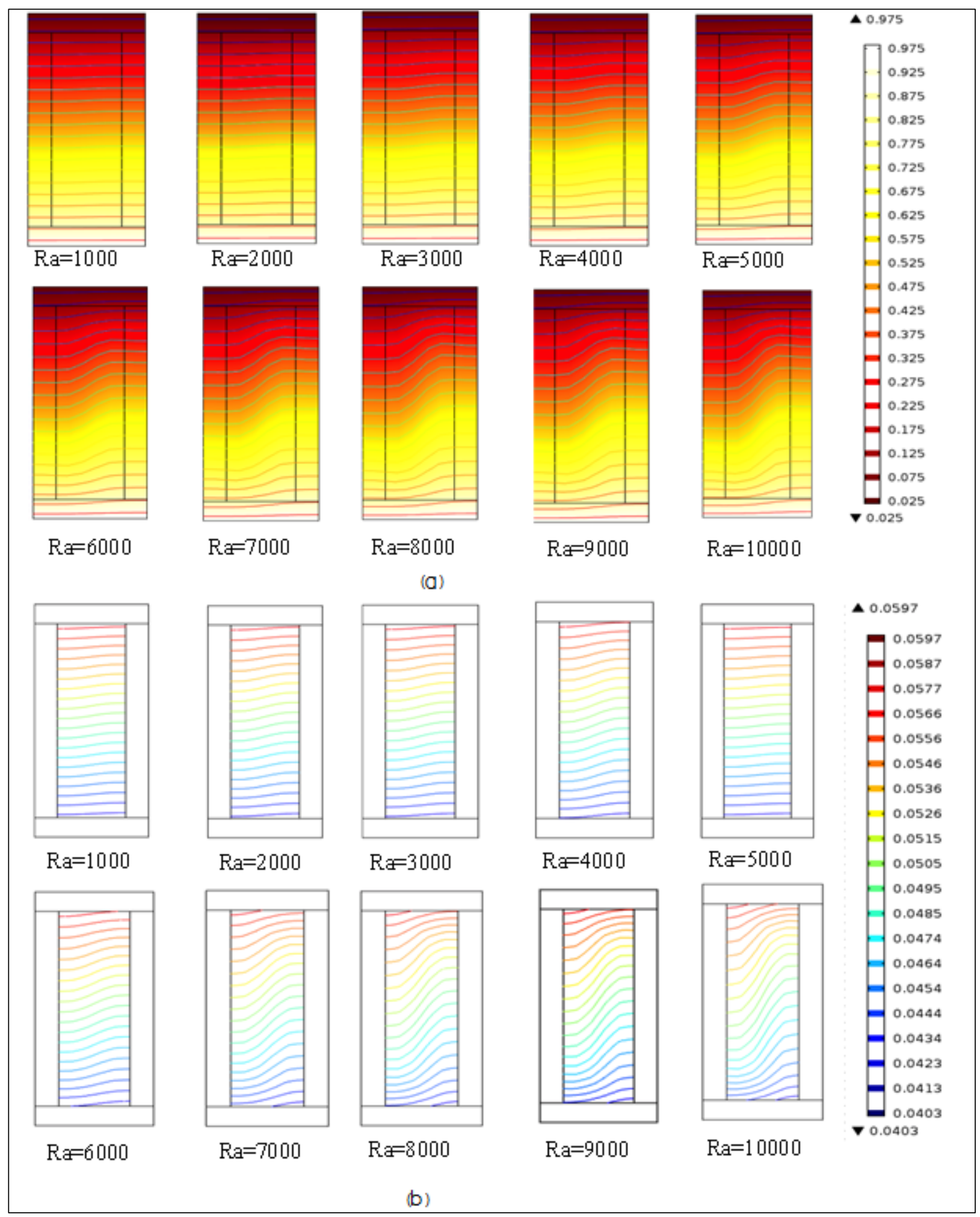

Figure 4 - 4: Temperature and concentration distribution in the cell in bottom heating; (a) Constant temperature lines in the cavity; (b) Constant concentration lines in the cavity. 


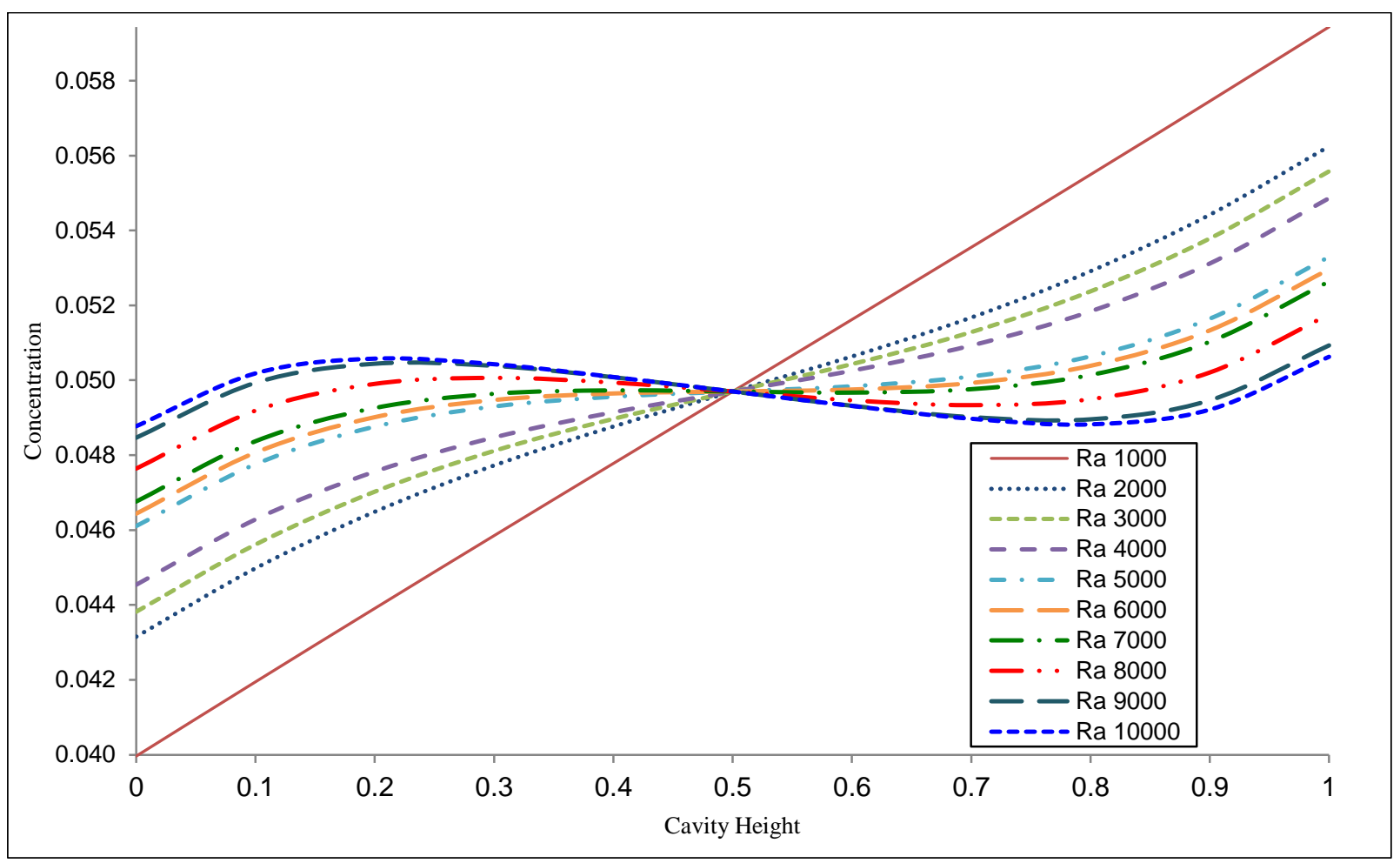

Figure 4 - 5: Variation of concentration at different Rayleigh numbers in bottom heating.

\subsection{Summary}

The focus of this chapter was to study the influence of natural convection and radiation in the presence and absence of thermodiffusion on binary molten metals alloys. The finite element method was used to simulate the temperature, velocity, and concentration distributions of a binary molten alloy of Sn-Bi in a quartz cavity. Here two specific cases were studied: One in which the cooling occurs on bottom surface and the other in which the cooling occurs on the top surface. External natural convection and surface radiation to the ambient were found to have insignificant effects on the distributions of concentration, temperature, and velocity. 


\section{CHAPTER FIVE}

\section{Influence of Thermodiffusion on the Solidification of Binary Molten Alloys Utilizing the Finite Element Method}

\subsection{Introduction}

This chapter explains the simulation of the solidification process of a series of binary molten metals. At first, finite element (FE) model was constructed using axisymmetric elements to represent a three-dimensional cylindrical cavity. The FE model was validated first against published work by simulating the results widely accepted in the research community. Once the model was validated, it was used to simulate the solidification process for tin-bismuth (Sn-Bi), tin-cadmium (Sn-Cd), tin-zinc (Sn-Zn), tin-lead (Sn-Pb), tin-gallium (Sn-Ga), and bismuth-lead $(\mathrm{Bi}-\mathrm{Pb})$ binary molten metal alloys to study the temperature, velocity, and concentration distributions in the presence and absence of the newly proposed thermodiffusion factor.

The outline of this chapter is as follows: first, the tin-bismuth (Sn-Bi) alloy is modeled and the importance of this binary alloy is briefly explained. Then, the natural convection in molten metals, the effect of thermodiffusion, governing equations, numerical models, the boundary conditions, and results for $\mathrm{Sn}-\mathrm{Bi}$ is presented. Finally, using the same axisymmetric finite element (FE) model, solidification processes for the remaining five alloys were studied and the results are explained and discussed. 


\subsection{Tin-Bismuth (Sn-Bi) Alloy}

Amongst various alloys, Sn-Pb is most extensively used for electronic packaging. Nevertheless, because $\mathrm{Pb}$ causes environmental pollution, $\mathrm{Pb}$-free solders such as $\mathrm{Sn}-\mathrm{Bi}, \mathrm{Sn}-\mathrm{Ag}$, and $\mathrm{Sn}-\mathrm{Zn}$ alloys have been considered to substitute for Sn-Pb solders. Sn-Bi, Sn-Ag, and Sn-Zn eutectic and near eutectic alloys were previously used in some industrial applications for their particular advantages such as lower process temperatures, compatibility with specific substrates, or higher reliability under particular conditions. Particularly, Sn-Bi solder is a low melting point lead-free solder designed for use in soldering processes [174-176]. The low melting point of this solder makes it appropriate for soldering temperature-sensitive compositions and substrates. Moreover, when compared to the Sn-Pb solder, the $\mathrm{Sn}-\mathrm{Bi}$ solder possesses a greater strength and higher creep resistance, but it has a lower ductility.

For the duration of soldering, the solder alloy melts and therefore reacts with the substrate to develop intermetallic compounds at the mutual interface. The growing of interfacial reaction products across solid-state aging in solder junctions is of specific attention to the

electronics industry. Extremely thick reaction layers, which develop between solder and substrate can deteriorate the physical and mechanical properties of solder junctions, specifically in high-influence load environments. Therefore, it is vital to understand and control the factors that control the kinetics of interfacial reactions [177-180].

Since this section focuses on the solidification of molten metal alloys, it is necessary to study natural convection and thermodiffusion in binary liquid alloys in more detail.

\subsection{Natural Convection in Liquid Metals}

Convective flow in the liquid phase in the vicinity of a growing solid has been discovered 
to have a large effect on the arrangement and composition of the solidified layer. The shape of the convection in the molten metal during solidification is similar to that of the convection in a liquid without a phase change. There are two variables in the convective flow: the changing temperature and the concentration gradient. As the density of most fluids changes with temperature, temperature gradients in the melt regularly cause density gradients. Therefore, solute convective flow happens with a concentration variation that increases from solute rejection at the solid-liquid interface. Therefore, the system is unstable and the flow in the liquid influences the heat and mass that move to the solid-liquid interface. This type of flow is assumed to be the reason for the macro- and microsegregation that affect the mechanical properties of the resultant solid metal. There have been studies that show the fluid motion in the liquid has a big effect on crystal growth, and it can also cause considerable changes in the form and properties of the resultant crystals [181].

Natural convection throughout solidification causes macrosegregation formations in castings and affects properties such as the size, orientation, and distribution of grain morphology, all of which influence the degree of variability in material properties. Improvements to solidification technologies rely on a better understanding of convective transport phenomena during phase changes and on the improvement of simulation tools. Owing to the release or absorption of latent heat during solidification or melting, most phase change problems are nonlinear. Exact solutions are only accessible for one-dimensional pure materials [182]. It is also challenging to carry out experiments that can simulate the exact conditions that take place in metal processing or crystal growth processes. As most of the liquid metal is opaque, it is almost impossible to measure the flow pattern within the melt and the liquid-solid phase contact via conventional methods such as particle image tracing techniques [1]. 


\subsection{Effect of Thermodiffusion on Solidification}

Currently, nonlinear models are used for many different cases of solidification, but they do not consider the Soret effect and temperature-dependent diffusion. Nevertheless, as the liquid temperature gradient is often high, thermodiffusion (the Soret effect) must be considered as well. The diffusion flux controls the solute gradient in the liquid at a certain growth rate, and consequently it also controls the value of the essential super cooling. In numerous cases, thermodiffusion may have a strong dependency on composition. For dilute solutions, the thermodiffusion flux is related to the average concentration as below [45]:

$\vec{J}=-D \nabla C-D_{T}\left(c_{0}+c_{1} C\right) \Delta T$

where $C$ and $T$ are the concentration and temperature fields, $D$ is the molecular diffusion coefficient, $D_{T}$ is the thermodiffusion coefficient, $c_{0}$ and $c_{1}$ are constants standing for initial concentrations.

The Dufour effect, where concentration gradients affect temperature, is reciprocal to the Soret effect, in which temperature gradients alter concentration. Similarly to the Soret effect, the Dufour effect is also identified from irreversible thermodynamics. However, in binary liquid alloys the Dufour effect is insignificant (this effect is most important in gas mixtures) [183]. In general, the diffusion coefficient is not a constant with respect to temperature. This function is written in a linear form as follows [184]:

$D(T)=D_{0}+\frac{\partial D}{\partial T}\left(T-T_{m}\right)$ 
where $D_{0}$ is the molecular diffusion coefficient at the melting temperature $T_{m}$ and $\frac{\partial D}{\partial T}$ is the temperature coefficient. Further, in accordance with nonlinear transport, Eqs. 5.1 and 5.2 will be used to analyze solidification processes with a mushy zone. It will also consider solidification models that include the Soret effect and temperature-dependent diffusion by cooling from below.

Currently, despite the comprehensive history of the study of solidification, many features of the physics of this phenomenon remain ambiguous. There is no other work for various cases that consider the Soret effect and temperature-dependent diffusion numerically for binary molten metal alloys. First, it was studied the solidification of Sn-Bi in a cavity, by solving the heat transfer, the fluid flow, and species equations using the finite element (FE) in the presence of thermodiffusion. To the best of our knowledge, these important details about the thermodiffusion effect on solidification of binary molten metals have not been studied. Thus, it was focused our attention on addressing the effect of thermodiffusion and material properties on the solidification process of binary molten metals.

\subsection{Governing Equations}

To attain accurate results, the model of the melt flow field should contain the heat transfer and phase change, the momentum, the continuity, and mass transport equations. The model included the phase change from liquid to solid, both in regards to latent heat and to variable physical properties. Due to the existence of an axis of symmetry in the boundary conditions, and to reduce the computational time without loss of accuracy and generality in the present study, an axisymmetric formulation was adopted.

The governing equations of composition given in Eqs. 5.3 to 5.16 were used to model the mushy region (Figure 5-1). This composition combines liquid and solid and is quantified by their 
volume fractions $f_{l}$ and $f_{s}$, respectively. The liquid molten metal alloy changes from a purely liquid to a purely solid region. Solidification appears in front of the cold area such that the solid phase is stationary and attaches to cold surface.

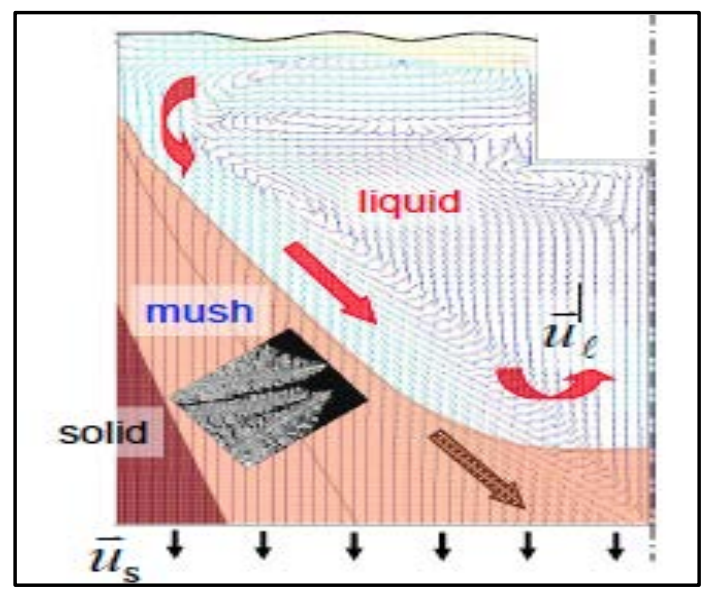

Figure 5 - 1: Schematic of the two-phase nature of a solidifying mushy region [106].

In geometry, the model used was a cylindrical cavity containing the $35 \% \mathrm{Bi}$ and $65 \%$ of $\mathrm{Sn}$. Our model for the vertical solidification accounts for the solidification of the liquid phase, containing convection and conductive heat transfer with a mushy region phase transition. The continuum model was implemented for simulating the solidification of the binary molten metal alloys under the following assumptions:

- All of the properties of the binary molten metal alloys can be found from the properties of the components of each phase.

- The density is steady in each phase but the densities of the two phases are dissimilar.

- The mushy region is modeled by means of an isotropic permeability method.

- The phases are in local thermodynamic equilibrium. The phase diagram is employed. 
- The buoyancy force is represented by means of the Boussinesq approximation.

- The flow of the liquid phase is laminar.

The heat transport is explained by the following equation [98]:

$\frac{\partial}{\partial t}\left(\rho c_{p} T\right)+\frac{1}{r} \frac{\partial}{\partial r}\left(\rho c_{p} u T_{r}\right)+\frac{\partial}{\partial z}\left(\rho v c_{p} T\right)=\frac{1}{r} \frac{\partial}{\partial r}\left(k r \frac{\partial T}{\partial r}\right)+\frac{\partial}{\partial z}\left(k \frac{\partial T}{\partial z}\right)+S_{e}$

where $k, \rho$, and $c_{p}$ denotes thermal conduction, density, and specific heat, respectively. The heat capacity in the mushy zone is not a constant; it is instead described by the following modified equation [90]:

$c_{p}=175-3.2 \operatorname{Heav}\left(T-T_{m} ; \Delta T\right)+L . \delta$

where the last term is taken into account for the release of the latent heat of fusion $L$. $\delta$ being a Gaussian curve defined as below [90]:

$\delta=\frac{\exp \left(-\frac{\left(T-T_{m}\right)^{2}}{\Delta T^{2}}\right)}{\Delta T \sqrt{\pi}}$

the function $\operatorname{Heav}\left(x-x_{m} ; \Delta x\right)$ shown in Eq. 5.4 is a smooth Heaviside step function defined as [172]:

$\operatorname{Heav}\left(x-x_{m} ; \Delta x\right)=0.5\left[1+\tanh \left(\frac{x-x_{m}}{\Delta x}\right)\right]$ 
where $\Delta x$ is the transition with between 0 and $1, x_{m}$ is in the middle of this interval, and $T_{m}$ in Eq. 5.5 is the melting temperature. A similar form of the Heaviside function was applied when the temperature dependence of the density and thermal conductivity was taken into account.

The source terms in the energy equation (Eq. 5.3) are given by following equation [98]:

$S_{e}=L \frac{\partial}{\partial t}\left(\rho f_{s}\right)-\frac{\partial}{\partial t}\left(\rho f_{s}\left(c_{s}-c_{l}\right) T\right)$

the source term of the solute equation is reliant on whether primary or secondary solidification is in progress. $f_{s}$ and $f_{l}$ are fractions of solid and liquid in the mixture, respectively. Using the Navier-Stokes equation in the FE simulation solves for the fluid flow, given by the following equations [136]:

$r$-momentum equation (for radial coordinates)

$\rho \frac{\partial u}{\partial t}+\frac{1}{r} \frac{\partial}{\partial r}(\rho u u r)+\frac{\partial}{\partial z}(\rho u v)=\frac{1}{r} \frac{\partial}{\partial r}\left(\mu r \frac{\partial u}{\partial r}\right)+\frac{\partial}{\partial z}\left(\mu \frac{\partial u}{\partial z}\right)-\frac{\partial P}{\partial r}-R_{r}$

z-momentum equation (for axial coordinates)

$\rho \frac{\partial v}{\partial t}+\frac{1}{r} \frac{\partial}{\partial r}(\rho u v r)+\frac{\partial}{\partial z}(\rho v v)=\frac{1}{r} \frac{\partial}{\partial r}\left(\mu r \frac{\partial v}{\partial r}\right)+\frac{\partial}{\partial z}\left(\mu \frac{\partial v}{\partial z}\right)-\frac{\partial P}{\partial z}-R_{z}-B_{z}$

in the above equations, $r$ is the radial coordinate on the horizontal axis and $z$ is the axial coordinate on the vertical axis, $t$ is time, $u$ and $v$ are the velocity components along the $r$ and $z$ coordinates, respectively, $T$ is temperature, $\rho$ is density, $\mu$ is viscosity, $P$ is pressure, $R_{r}$ and $R_{z}$ are the Darcian resistance terms, and $B_{z}$ represents the body force. 
The Darcian resistance terms and the body force were used in our model for two reasons: a) To diminish the velocity at the phase-change interface such that the velocity of the liquid at the interface is equal to that of the solidified phase after transition, and b) to include the effects of buoyancy. The solidification model was borrowed directly from the model suggested by Voller and Prakash [102]. The above governing equations are in an unspecified format for the numerical solution of heat and fluid flow problems that include a transient term, a diffusive term, a convective term, and a sources term. In this form, the solution to the above problem is achieved by the description of the source terms. The $R_{r}$ and $R_{z}$ source terms were applied to adjust the momentum equations in the mushy region. These Darcy sources were applied for modelling the influence of the properties of the porosity of the mushy region on the flow field. If it is assumed that the Darcy law governs the flow in the mushy region [102], then

$$
u=-\left(\frac{k}{\mu}\right) \operatorname{grad} P
$$

such that $k$, the permeability, is a function of the porosity. When the porosity decreases, the permeability and the surface velocity also decrease, down to a value of zero once the mushy zone becomes completely solid. In a numerical model this behaviour can be explained by defining sources as follows:

$R_{r}=-A v \quad$ and $\quad R_{z}=-A w$

Here $A$ increases from zero to a large value, while the local solid fraction $f_{s}$ increases from its liquid value of 0 to its solid value of 1 . 
In the liquid region, the sources get a zero value and the momentum equations are in terms of real fluid velocities. In the mushy region the value of $A$ increases when the value of the sources start to control the transient, convective, and diffusive terms and when the momentum equation estimates the Darcy law. When the local solid fraction reaches 1, the sources govern all other terms in the momentum equation. This consequently forces the expected surface velocities to have values close to zero. For a mushy region phase change, where a porous region is present, one can demand a derivation of an appropriate form for the function $A$. A well-known equation extracted from the Darcy law is the Carman-Koseny equation [185]:

$$
A=-C \frac{\left(1-f_{l}\right)^{2}}{\left(f_{l}^{3}+\varepsilon\right)}
$$

Where $f_{l}$ is the volume fraction of the liquid phase and $A$ and $\mathcal{E}$ represent arbitrary constants ( $A$ should be large and $\varepsilon$ small to give a proper damping). The fraction of the liquid phase $f_{l}$ is given by the following equation [119]:

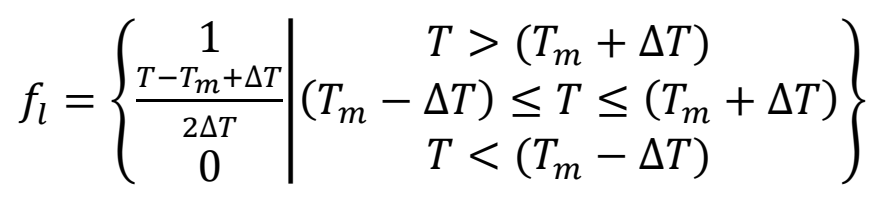

The value of $C$ was determined by the morphology of the porous media. In the current study, $C$ was presumed constant and was set to $1.6 \times 10^{3}$. The constant $\varepsilon$ was fixed at 0.001 , and it was present to prevent division by zero. Note that this $\varepsilon$ in Eq. (5.12) is not the porosity, rather, it is an arbitrary number used for the numerical stability of the FE solution. It may be noted that 
the selected value of $C$ is large enough to allow for considerable flow in the mushy region at low local solid fractions. However, the limiting value of $A$, i.e., $-\frac{C}{q}$, is large enough to dominate the fluid velocities in the solid. This will have the effect of diminishing the flow in the mushy region at full solidification. By using the Boussinesq approximation, the body force in the $z$-momentum equation is defined as follows [136]:

$B_{z}=\rho_{0} g_{z}\left[1-\beta_{T}\left(T-T_{r e f}\right)-\beta_{C}\left(C-C_{r e f}\right)\right]$

where $\rho_{0}$ is the density of the liquid at the reference temperature $\left(T_{r e f}\right)$ and the reference solute concentration $\left(C_{r e f}\right)$ and $\beta_{T}$ and $\beta_{C}$ are the thermal and the solutal expansion coefficients, respectively. Other equations used in the simulation were continuity conditions and the mass transport equation, and they are defined as follows [136]:

Continuity:

$\frac{1}{r} \frac{\partial}{\partial r}(\rho u r)+\frac{\partial}{\partial z}(\rho v)=0$

Mass transport:

$\frac{\partial}{\partial t}(\rho C)+\frac{1}{r} \frac{\partial}{\partial r}(\rho u r C)+\frac{\partial}{\partial z}(\rho v r C)=\frac{1}{r} \frac{\partial}{\partial r}\left(\rho D_{m} \frac{\partial C}{\partial r}\right)+\frac{\partial}{\partial z}\left(\rho D_{m} \frac{\partial C}{\partial z}\right)+\frac{1}{r} \frac{\partial}{\partial r}\left(\rho D_{T} \frac{\partial T}{\partial r}\right)+$

$\frac{\partial}{\partial z}\left(\rho D_{T} \frac{\partial T}{\partial z}\right)$

Where $C$ is solute concentration, $D_{m}$ is molecular diffusion coefficient, and $D_{T}$ is the thermodiffusion coefficient. The melt solidifies as it cools down in the cavity. This phase change 
releases latent heat, which the model includes. Moreover, for molten metal alloys, the phase change is often spread out over a temperature range. As the liquid metal solidifies, the material properties change significantly. Finally, the model also contains the "mushy region" a combination of solid and molten material that exists due to a somewhat vast transition temperature of the alloy and the solidification kinetics.

\subsection{Numerical Model and Boundary Condition}

The numerical model was implemented using the commercial software COMSOL4.3b, The case under study corresponds to a cylindrical cavity with $100 \mathrm{~mm}$ height and $25 \mathrm{~mm}$ radius, which contains a Sn-Bi mixture that solidifies by cooling from the bottom.

Figure 5-2 shows the geometry of the cylindrical cavity filled with the binary molten metal. It also shows the boundary conditions that were implemented into the FE model. The imposed boundary conditions that were used in the FE models were as follows: the top of the cavity was the hot surface and the bottom was the cooled surface. The vertical walls were adiabatic. The radial heat transfer at the outer surface of the cylinder was taken to be zero. At all boundaries, the mass flux was zero. Along the line of symmetry, the gradients of velocities were taken to be zero. The numerical values for the properties of the Sn-Bi mixture used in the simulation are summarized in Table 5-1. 


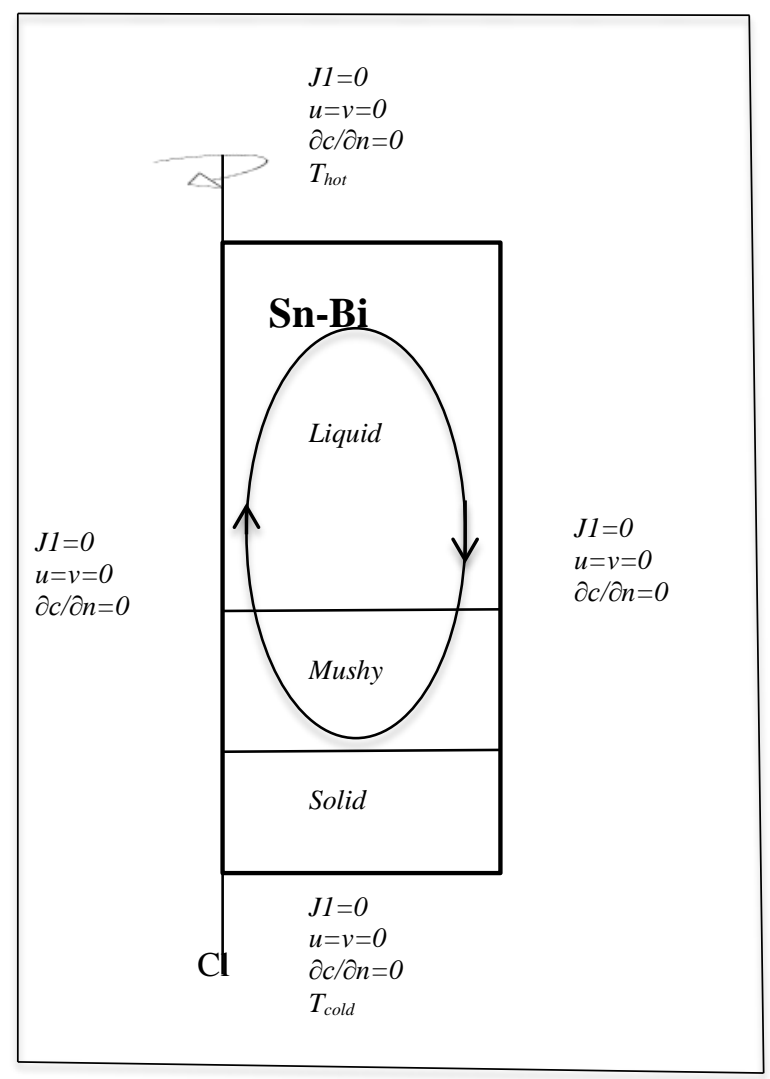

Figure 5 - 2: Schematic diagram of the model for molten alloy. 
Table 5 - 1: The Physical Properties of Sn-Bi Molten alloy at $235{ }^{\circ} \mathrm{C}$ [25]

\begin{tabular}{|c|c|c|}
\hline Physical Properties & Symbols & Sn-Bi \\
\hline Initial Concentration for $\mathrm{Bi}$ & $\mathrm{C}_{\mathrm{i}}$ & 0.35 \\
\hline Dynamic Viscosity & $\mu$ & $15.76 \times 10^{-3}$ g.cm $\mathrm{cm}^{-1} \cdot \mathrm{s}^{-1}$ \\
\hline Density & $\rho$ & 7.902 g.cm ${ }^{-3}$ \\
\hline Hot Temp & $\mathrm{T}_{\mathrm{H}}$ & $300^{\circ} \mathrm{C}$ \\
\hline Cold Temp & $\mathrm{T}_{\mathrm{C}}$ & $166^{\circ} \mathrm{C}$ \\
\hline Conductivity & K & $0.4707 \mathrm{~J} . \mathrm{s}^{-1} \cdot \mathrm{cm}^{-1} \cdot \mathrm{k}^{-1}$ \\
\hline Specific Heat Capacity & $\mathrm{C}_{\mathrm{p}}$ & $0.19025 \mathrm{~J}^{-g^{-1}} \cdot \mathrm{k}^{-1}$ \\
\hline Temperature of eutectic reaction & $\mathrm{T}_{\text {eutectic }}$ & $139^{\circ} \mathrm{C}$ \\
\hline Solutal Expansion & $B c$ & $48.02 \times 10^{-4}$ \\
\hline Thermal volume expansion & $\beta_{T}$ & $1.01 \times 10-^{4}\left(\mathrm{~K}^{-1}\right)$ \\
\hline Kinematic Viscosity & $v$ & $1.994 \times 10^{-3} \mathrm{~cm}^{2} \cdot \mathrm{s}^{-1}$ \\
\hline Thermal Diffusivity & $\alpha$ & $0.3132 \mathrm{~cm}^{2} \cdot \mathrm{s}^{-1}$ \\
\hline Characteristic Velocity & $u_{o}$ & $0.2622 \mathrm{~cm} . \mathrm{s}^{-1}$ \\
\hline Eutectic concentration for Sn & $\mathrm{C}_{\mathrm{e}}$ & 0.56 \\
\hline Soret Coefficient & $S_{T}$ & $0.243\left(\mathrm{~K}^{-1}\right)$ \\
\hline Melt point of pure solvent (Sn) & $\mathrm{T}_{\mathrm{m}}$ & $231.9^{\circ} \mathrm{C}$ \\
\hline Thermodiffusion Factor & $\alpha_{T}$ & 0.141 \\
\hline
\end{tabular}

In the mushy region, a fluid of varying composition and density is slowly drawn through a network of dendrites by convection and solidification shrinkage. The solidus line, i.e., the temperature isotherm, shows a small deformation due to convection. In the region just above the mushy zone, a fully liquid metal pool undergoes a stronger convective flow. This flow is setup by temperature-induced density differences in an essentially homogeneous liquid. The flow direction, 
away from the cold wall, increases the heat loss in this area and hence extends the mushy region. The flow in the mushy area will be controlled by the presumed nature of the porosity-permeability relationship [180]. The phase diagram of the Sn-Bi system is shown in Figure 5-3. For the Sn-rich alloy, the density of the liquid increases with increasing solute content in the mushy zone as solidification proceeds from the liquidus to the solidus. The temperature $T$ and the solute concentration $C$ are related by the phase diagram.

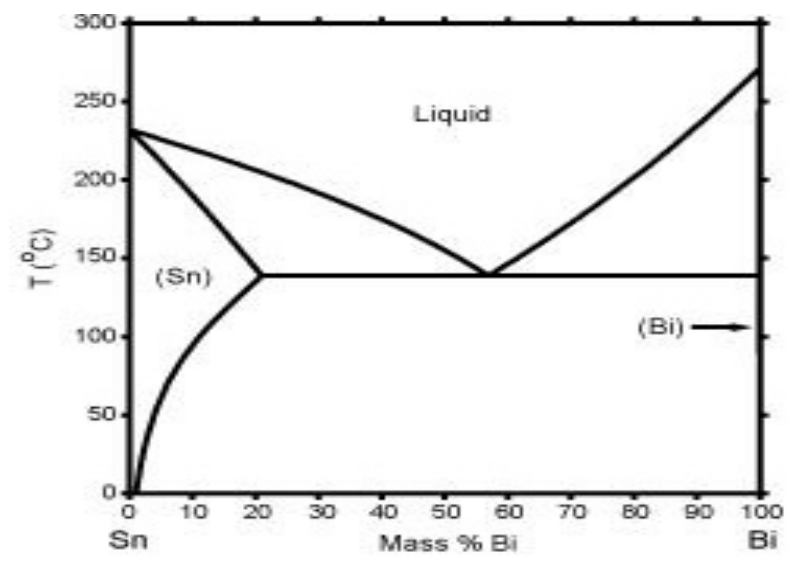

Figure 5 - 3: Sn-Bi equilibrium phase diagram [180].

\subsection{Results and Discussion}

The first step of this research was to validate the FE model. It was achieved by reproducing a previously solved problem, that of Singh et al. [143], and comparing the results. At first, it was used identical materials, boundary conditions, and governing equations as were used by Singh and co-workers. The results of this simulation were similar to the published results indicating our model performed correctly. Figure 5-4 and Figure 5-5 depict the comparison of temperatures and compositions of this study. 


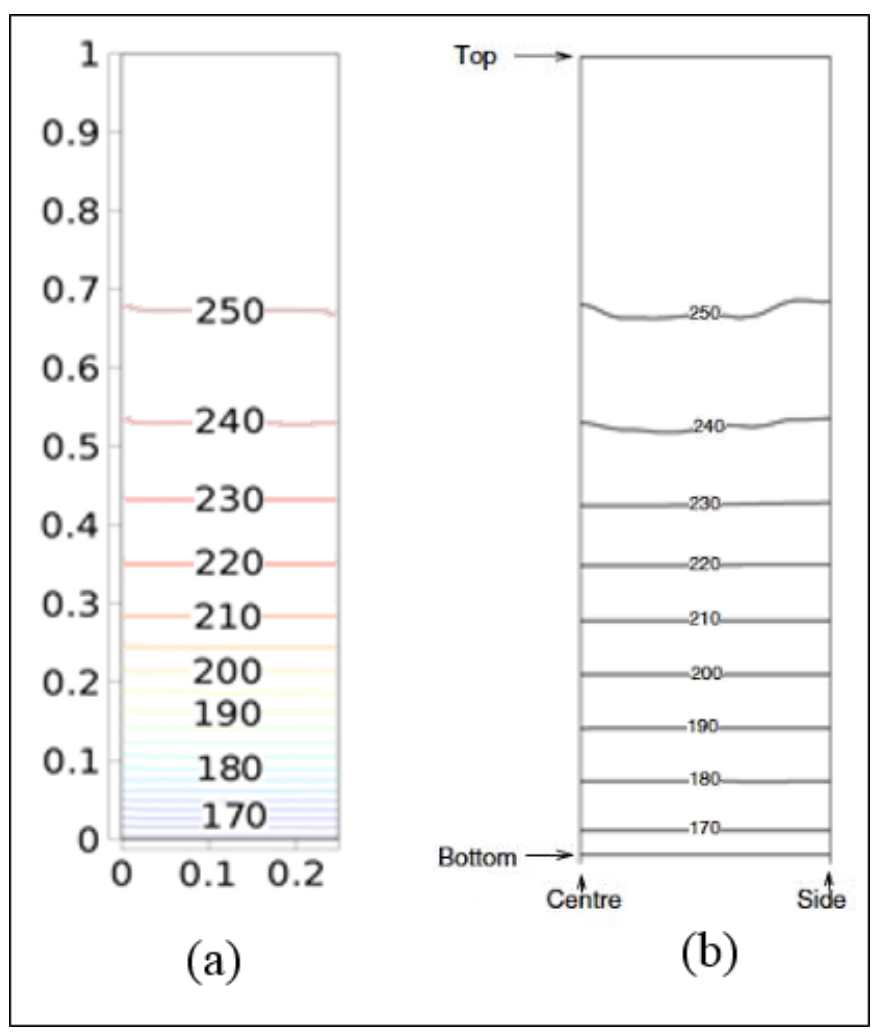

Figure 5 - 4: Simulated result for temperature distribution at 25\% solidification of Pb-0.35Sn; (a) Our reproduced simulation, (b) Extracted from published research.

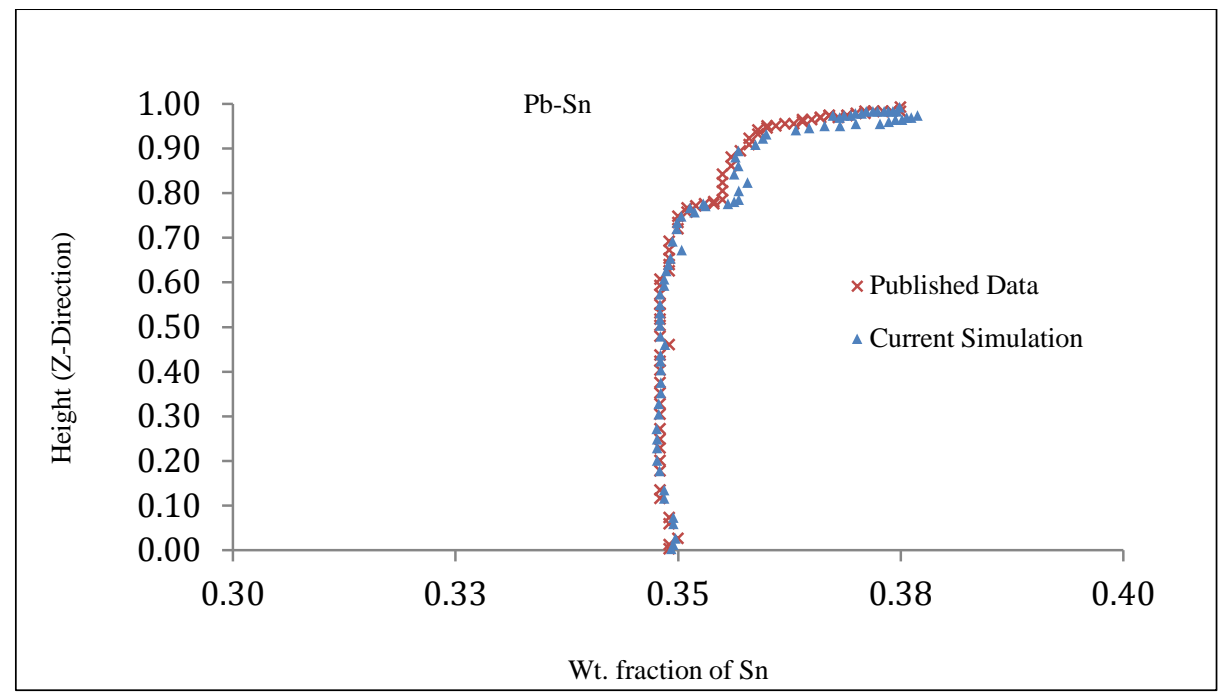

Figure 5 - 5: Comparison of the simulated result for composition distribution at 25\% solidification of $\mathrm{Pb}-$ 0.35 Sn. 
Figure 5-5 shows the comparison of the current simulation and that of Singh et al. [143]. It is clear that except for the transition area between the liquid and mushy zone that shows a very small discrepancy, the simulation is identical to the published data. The small discrepancy at the liquid to mushy transition zone could be attributed to type of solver, or the size and number of the element and mesh size. Subsequently, it was used current FE models and applied materials and boundary conditions specific to the simulation of interest. At this point the effect of thermodiffusion was also included in the model. As previously explained in chapter 4, the same mesh sensitivity analysis was performed to ensure the results are independent to the mesh size to within $1 \%$ tolerance. Furthermore, the same number of iteration and convergence tolerance as explained in chapter 4 were applied to simulation of solidification.

The porous media-based formulation was used for vertical solidification; the results of these simulations are presented in terms of velocity, temperature distribution, fraction of liquid phase, and concentration. The most important results of these studies are that flow in the mushy region is always driven by flow in the bulk liquid and that a proper model for permeability is crucial if the author hope to obtain realistic simulations of solidification. Our preliminary results suggest that the nature of the porosity has a significant effect. Simulations were carried out with the Carman-Kozeny [185] permeability model. The authors show that the fluid flow developed in the liquid metal ahead of the liquidus isotherm under steady-state conditions. It is also shown that natural convection has little effect on the mushy zone fluid flow and changes in the composition. In bottom-cooled solidification, the temperature increases in the upward direction, and, in most cases, the liquid is denser towards the bottom. Hence, the thermal force opposes the upward motion of the liquid. It should be noted that the thermal force and concentration force oppose each 
other near the mushy-liquid interface, which leads to the reduction of the net flow strength and consequently the variation of concentration.

For binary systems where the thermodiffusion coefficient of solute is positive, $D_{T}>0$, and rejected solute in the mushy region is heavier than the solvent, the solutal force acts downward making both thermal and solutal forces stable. Thermosolutal convection then occurs due to liquid build up ahead of a growing solid-liquid interface in the Sn-Bi alloy. An alloy with a lighter solute has the solutal force acting in an upward direction and during bottom-cooled solidification molten liquid can become unstable.

However, by increasing the temperature gradient, the bulk fraction of the solid increases and consequently causes the mushy zone thickness to increase. In addition, a positive or negative value of $D_{T}$ increases or decreases the solidified fraction in mushy region, respectively [139]. In the case of $D_{T}<0$, the mass fluxes caused by the Soret effect and normal diffusion have the same direction. That is, the mass outlet from the mushy region becomes greater and increases the temperature. In the case of $D_{T}>0$, both fluxes act in opposite directions, and, as a consequence, thermodiffusion assists the growth of the solid fraction. As the solidification progresses, the flow field gradually shifts towards the hot wall of the cavity and weakens the flow strength due to the spread of the mushy region. When solidification has completed in the cavity, there is no flow remaining. The preceding simulation was carried out for two cases, one with and one without thermodiffusion, to study the variation of velocity, temperature distribution, fraction of the liquid phase, and concentration gradient for both cases.

\subsubsection{Results Excluding the Effect of Thermodiffusion}

Figures 5-6(a) - 5-8(a) show the calculated profiles of various fields at $25 \%$ solidification. This solidification was chosen due to the fact that at $25 \%$ solidification the boundaries between 
the solid, mushy, and liquid zones were pronounced. These profiles are qualitatively similar to those with thermodiffusion. The flow was concentrated near the centre of the cavity and is in an upward direction. Thus, the strength of convection is practically negligible at a sufficient distance from the centre of the mushy region, as shown in Figure 5-6 (a). The temperature distribution is linear for the both solid and liquid regions, but it fluctuates in the mushy zone, as shown in Figure 5-7 (a). Figure 5-8 (a) shows the fraction of the liquid phase and the variation of the liquid fraction between the cold zone and the hot zone. Figure 5-8 (a) also shows the channelling effect that takes place at the boundary of the mushy and liquid zones starting from the centre of the cavity to the sidewall. The high concentration variation in the region surrounding the channel at the top central portion of the cavity is also shown.

The average composition variation distribution at the end of solidification in the vertical direction is shown in Figure 5-9. The concentration variation increased with a decrease in the solidification growth rate and in the mushy region length. The complex nature of the composition variation profile was attributed to complex thermosolutal convection in the mushy region. The minimum concentration for $\mathrm{Bi}$ was $34.46 \%$, whereas the maximum was $38.63 \%$. Both the maximum and minimum concentrations were located over a small zone of the channel at the top central part of the cavity. Towards the top of the cavity, there occurred the onset of channel formation, which appeared to form a combination of a local remelting zone in the solid-liquid region and caused dendritic growth around the remelted region of higher solute concentration.

\subsubsection{Results Including the Effect of Thermodiffusion}

Figures 5-6 (b) - 5-8 (b) present the velocity magnitude, the temperature distribution, and the fraction of the liquid phase, and Figure 5-9 shows the concentration distribution at $25 \%$ average solidification. Velocity streamlines of Figure 5-6 (b) were characterized by a weak 
upward flow near the left boundary. The velocity range had a higher value as compared to the simulation without thermodiffusion. Weak convection was responsible for the low level of concentration variation in the vertical solidification. The effect of flow on temperature distribution, Figure 5-7 (b), was small considering weak nature of the flow. The strength of the convection was not sufficient to bend the isotherm lines, but the fluctuation was present in the mushy region. Figure 5-8 (b) shows the fraction of the liquid phase in the mushy region, which was in the bottom half of the cylinder. The average variation of the composition in the vertical direction at the end of solidification is shown in Figure 5-9. Due to higher solidification forces and lower solidification time, the variation of composition was less in this case when compared to the simulation that did not include thermodiffusion, except near the top of cavity. The minimum concentration for $\mathrm{Bi}$ was $34.86 \%$, whereas the maximum was $37.95 \%$. In this case, variation of the concentration was practically non-existent for most of the solidified alloy.

\subsubsection{Comparison of the Results}

The above result clearly demonstrates the role of thermodiffusion on the evolution of the distribution of the composition. Weak flow caused local fluctuation in the composition pattern. However, large variations in concentration came through channel formation and supply of solute to the liquid region [143]. It should be noted that the variation in concentration was directly linked with the top portion of the mushy zone interacting with the liquid. The distribution and extent of concentration variation greatly depended on the nature of the convective flow in the mushy-liquid interface. For vertical solidification, the driving force for flow was small and was concentrated near the mushy-liquid interface [96, 143]. For bottom-cooling solidification, the dendrites aligned vertically, and there was a region near the dendritic tip free of branching, and thus highly 
permeable [143]. This permitted flow instabilities to grow. Such a highly permeable region near the mushy zone seemed crucial in changing of composition during vertical solidification.

The comparison of average composition variation at the end of solidification for the two cases showed that the case with thermodiffusion resulted in the minimum concentration variation. Conversely, for the case without thermodiffusion, a higher concentration variation was obtained. Our simulations show that relative changes in composition, as seen in Figure 5-9, caused by the Soret effect for minimum ranges of concentration between two cases was 1.16 and for maximum ranges was -1.76 . The relative change between the two cases is defined as follows:

Relative Change $=\frac{\text { Max } / \text { min value of } C \text { (thermodiffusion) }- \text { max } / \text { min of } C \text { (without thermodiffusion) }}{\max / \min \text { value of } C(\text { without thermodiffusion })} \times 100$

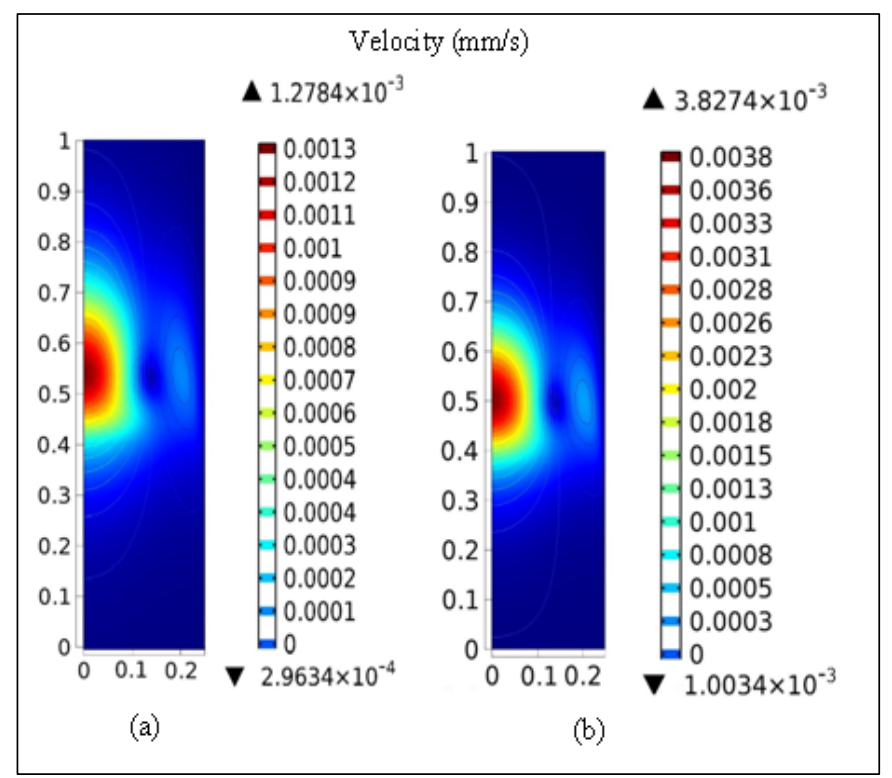

Figure 5 - 6: Simulated result for streamlines at $25 \%$ solidification of Sn-Bi; (a) without thermodiffusion simulation, (b) with thermodiffusion. 


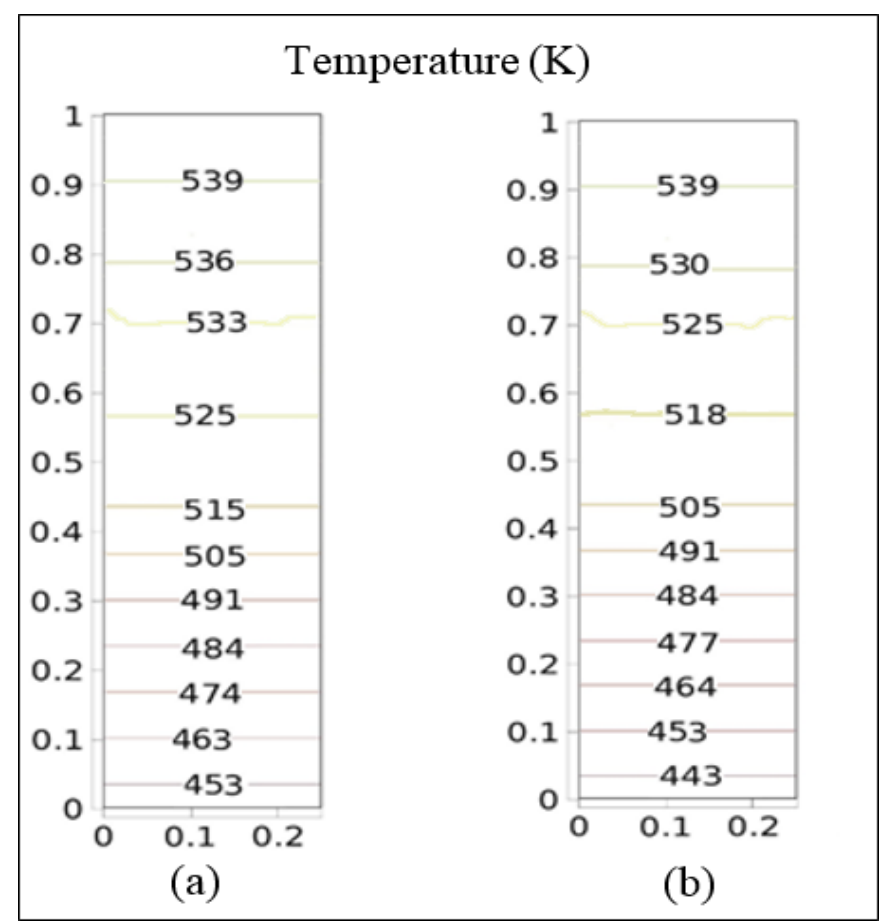

Figure 5 - 7: Simulated result for temperature distribution at $25 \%$ solidification of Sn-Bi; (a) without thermodiffusion simulation, (b) with thermodiffusion

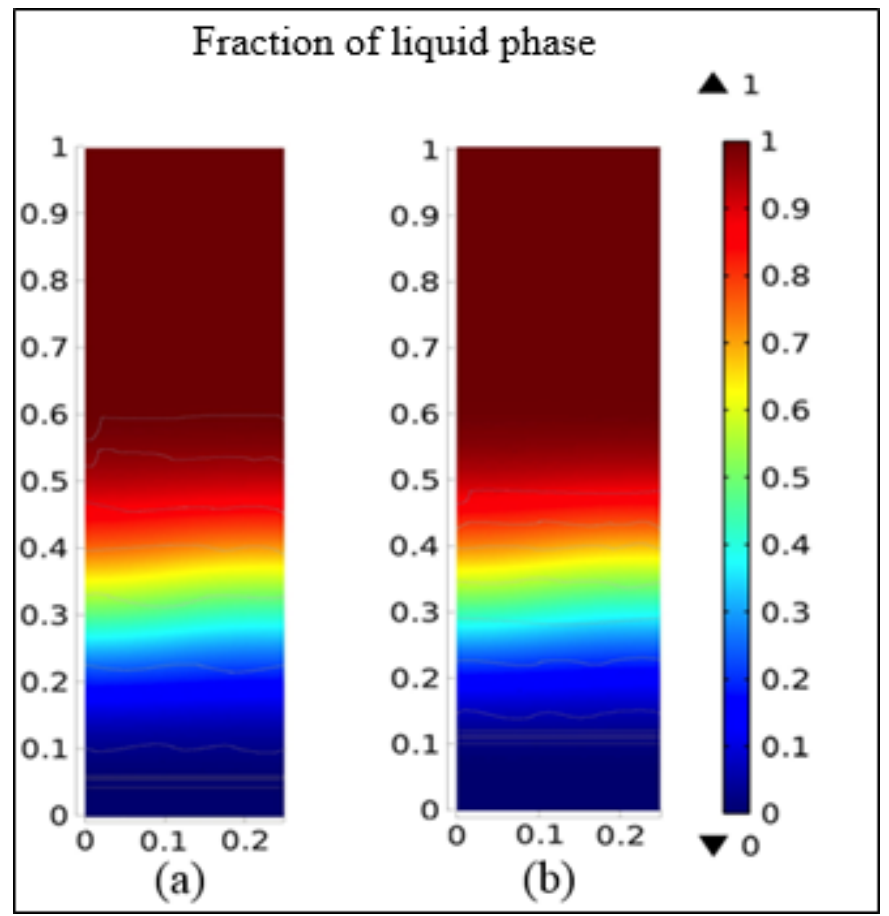

Figure 5 - 8: Simulated result for liquid fraction at $25 \%$ solidification of Sn-Bi; (a) without thermodiffusion simulation, (b) with thermodiffusion. 


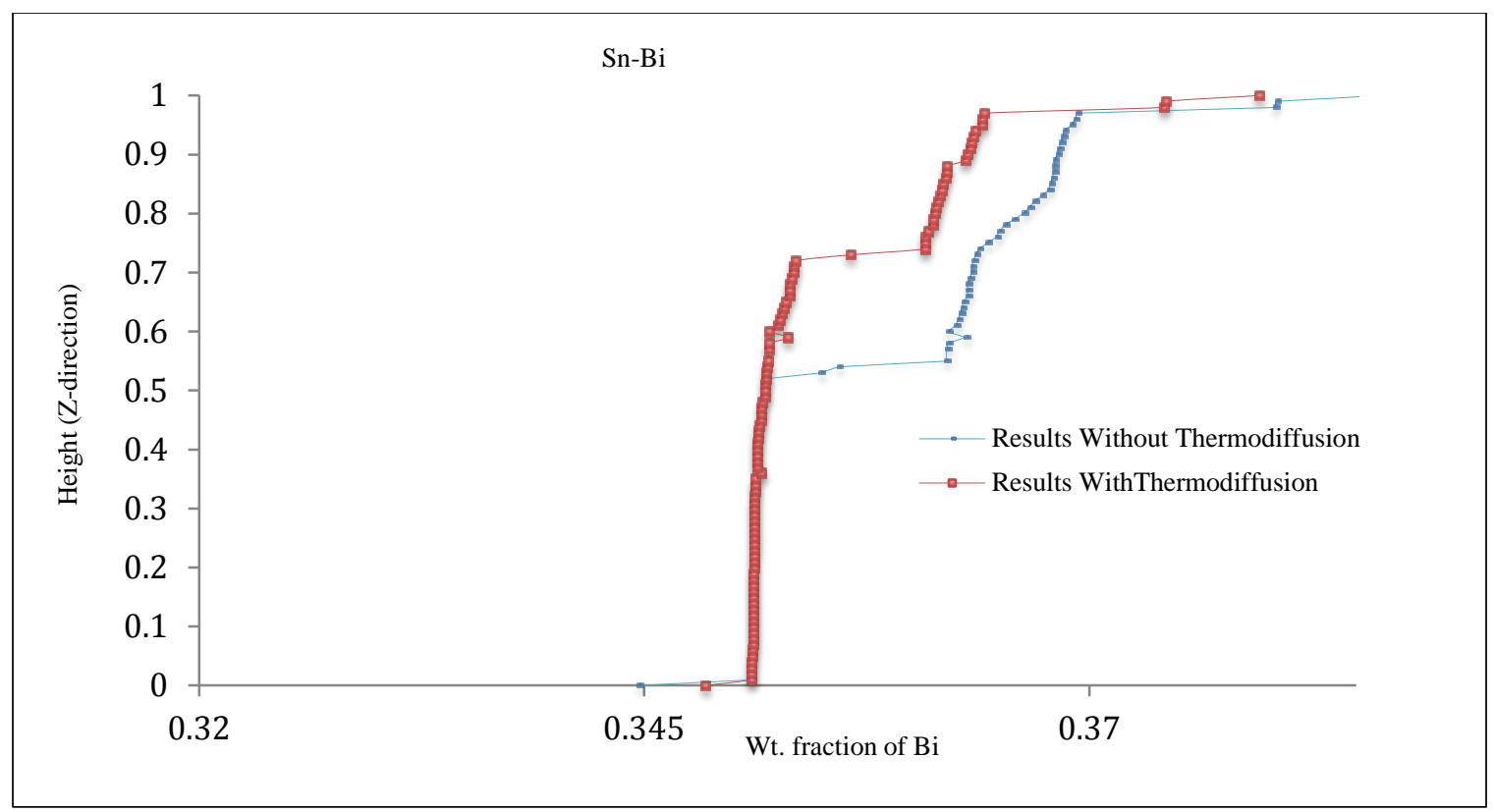

Figure 5 - 9: Simulated result for composition distribution at $25 \%$ solidification of Sn-Bi; (a) without thermodiffusion simulation, (b) with thermodiffusion.

Figure 5-10 shows the comparison of the temperature distribution calculated at a point 50 $\mathrm{mm}$ above the cooling zone for the two cases. The two distributions were identical in shape but have different solidification times. Thermodiffusion acted as a thermal force and promoted a faster cooling of the binary molten metal mixture. This naturally caused a faster solidification process. Since there was a redistribution of solute between the two phases during solidification, the temperature of the liquid varied as depicted in Figure 5-10. However, the effect of thermodiffusion was very small and resulted in an approximately $5 \%$ decrease in solidification time. Figure 5-11 shows the liquid fraction as a function of time for the two solidification simulations. Thermodiffusion played no role until the first layer of solid had been formed, at which time the model with thermodiffusion began to show faster solidification. In other words, the volume fraction of liquid in the model with thermodiffusion was smaller when compared to the model without thermodiffusion. 


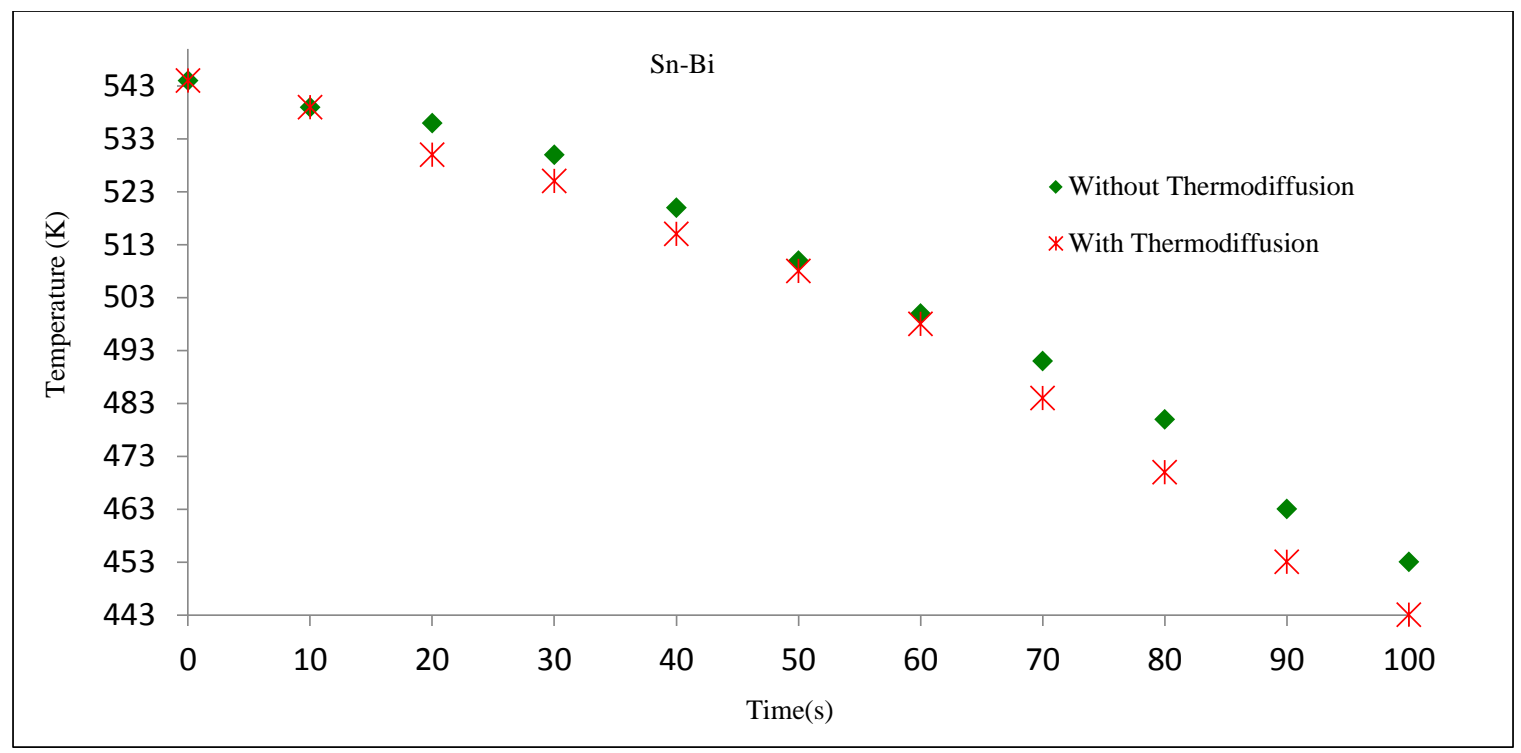

Figure 5 - 10: The time dependence temperature at the point $\mathrm{z}=50 \mathrm{~mm}$ above the bottom of the cavity for two cases; without /with thermodiffusion.

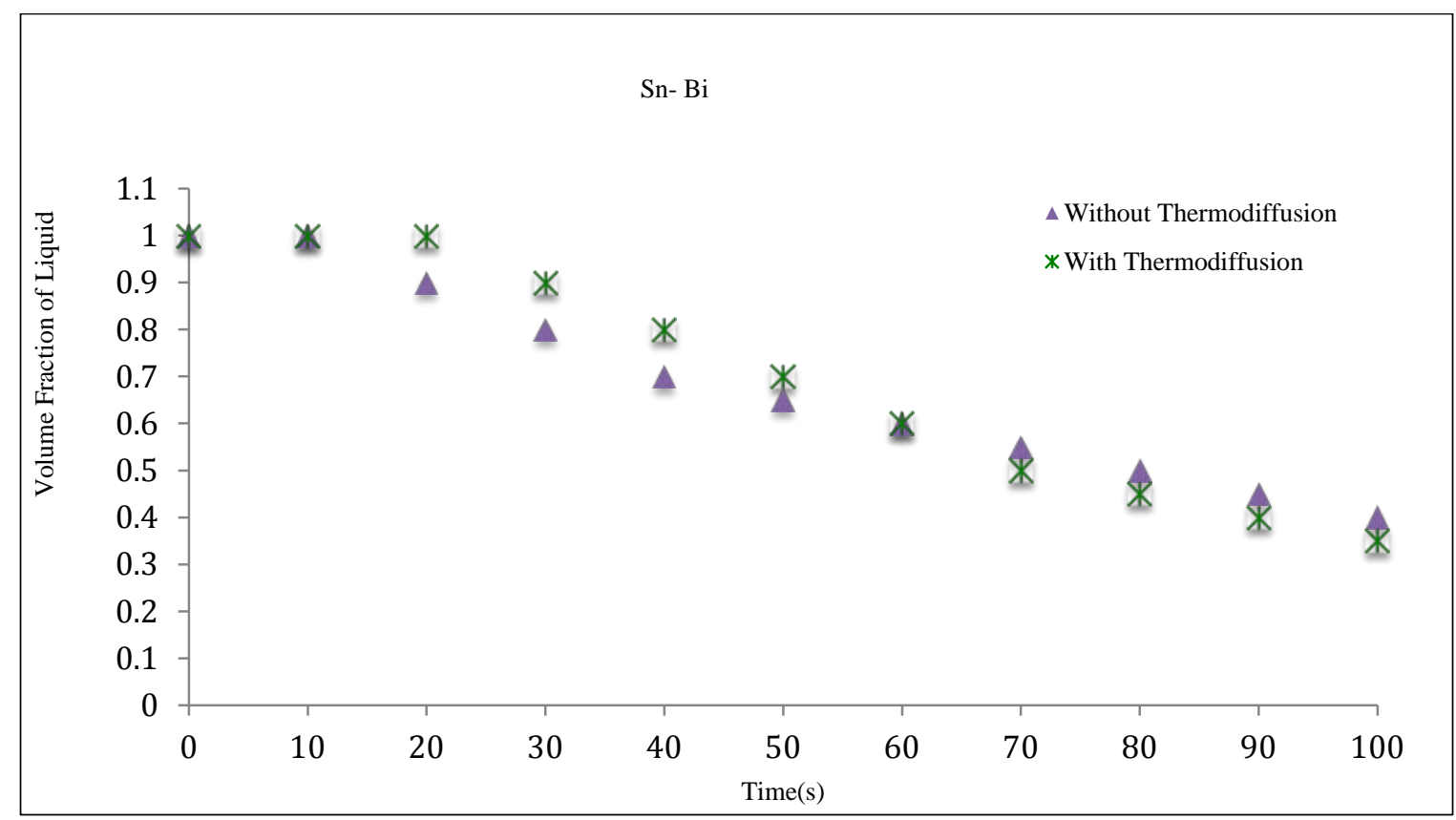

Figure 5 - 11: The line dependence of volume fraction of liquid phase at point $\mathrm{z}=50 \mathrm{~mm}$ above the bottom of the cavity for two cases; without /with thermodiffusion. 


\subsection{Influence of Material Properties on Solidification Progress}

Material properties and equilibrium phase diagrams govern thermosolutal convection in a significant way. Here a critical study of the role of material properties alone on the thermosolutal convection is presented by simulating solidification of tin-cadmium (Sn-Cd), tin-zinc (Sn-Zn), tinlead ( $\mathrm{Sn}-\mathrm{Pb})$, tin-gallium ( $\mathrm{Sn}-\mathrm{Ga})$, and bismuth-lead $(\mathrm{Bi}-\mathrm{Pb})$ binary systems in the presence of thermodiffusion. In all of the binary systems considered here, the solute is lighter than the solvent except for Sn-Ga. Thus, the thermodiffusion coefficients for Sn-Cd, Sn-Zn, Sn-Pb and Bi-Pb alloys are negative $\left(D_{T}<0\right)$. The physical properties pertaining to these systems are listed in Table 5-2. In order to carry out a meaningful comparative study, the geometry of the cavity and boundary conditions were kept identical for all of the binary materials considered here, including Sn-Bi. The initial concentration for these binary molten metals was selected based on Table 3-5 (in chapter 3). In Table 5-2, $N$ signifies the importance of the ratio of solutal buoyancy to thermal buoyancy in the mushy phase in the presence of solute rejection, and it is defined by the following expression [140]:

$N=\frac{\beta_{C}}{m \beta_{T}}$

where $m$ is the slope of the liquidus. It is important to note that the actual process is governed by the interaction of energy, momentum, and concentration transport.

\subsubsection{Simulation and Results}

Similar to the case of Sn-Bi, finite element simulations of the solidification process for SnCd, Sn-Zn, Sn-Pb, Bi-Pb, and Sn-Ga were carried out. The solidification simulations were 
performed for two cases, one with and one without thermodiffusion for all of the aforementioned binary molten alloys. Figures 5-12(a) to 5-16(a) show the simulations without thermodiffusion and Figures 5-12(b) to 5-16(b) show the simulations with the effect of thermodiffusion included.

Table 5 - 2: The Physical properties of binary molten alloys at $235^{\circ} \mathrm{C}$ [165]

\begin{tabular}{|c|c|c|c|c|c|c|}
\hline Physical Properties & Symbols & Sn-Cd & Sn-Zn & Sn-Ga & Sn-Pb & $\mathbf{B i}-\mathbf{P b}$ \\
\hline Initial Concentration & $\mathrm{C}_{\mathrm{i}}$ & 0.5 & 0.5 & 0.5 & 0.5 & 0.5 \\
\hline Dynamic Viscosity $\left(\mathrm{g} \cdot \mathrm{cm}^{-1} \cdot \mathrm{s}^{-1}\right)$ & $\mu$ & $21 \times 10^{-3}$ & $24 \times 10^{-3}$ & $11.5 \times 10^{-3}$ & $26.1 \times 10^{-3}$ & $9.42 \times 10^{-3}$ \\
\hline Density (g.cm ${ }^{-3}$ ) & $\rho$ & 7.46 & 6.74 & 6.41 & 10.6 & 10.56 \\
\hline Hot Temp (K) & $\mathrm{T}_{\mathrm{H}}$ & 573 & 573 & 573 & 573 & 573 \\
\hline Cold Temp (K) & $\mathrm{T}_{\mathrm{C}}$ & 439 & 439 & 439 & 439 & 439 \\
\hline Conductivity $\left(\mathrm{J} \cdot \mathrm{s}^{-1} \cdot \mathrm{cm}^{-1} \cdot \mathrm{k}^{-1}\right)$ & $\mathrm{K}$ & 0.823 & 0.92 & 0.543 & 0.2125 & 0.216 \\
\hline Specific Heat Capacity $\left(\mathrm{J} \cdot \mathrm{g}^{-1} \cdot \mathrm{k}^{-1}\right)$ & $\mathrm{C}_{\mathrm{p}}$ & 0.23 & 0.31 & 0.28 & 0.172 & 0.141 \\
\hline Temperature of eutectic reaction $(\mathrm{K})$ & $\mathrm{T}_{\text {eutectic }}$ & 449 & 472 & 302.78 & 456 & 397 \\
\hline Solutal Expansion & $\beta_{C}$ & $48.02 \times 10^{-6}$ & $3 \times 10^{-3}$ & $8.4 \times 10^{-5}$ & $0.364 \times 10^{-3}$ & $2.83 \times 10^{-2}$ \\
\hline Thermal volume expansion $\left(\mathrm{K}^{-1}\right)$ & $\beta_{T}$ & $31 \times 10^{-6}$ & $30.2 \times 10^{-5}$ & $9.85 \times 10^{-5}$ & $1.09 \times 10^{-4}$ & $21.15 \times 10^{-3}$ \\
\hline Kinematic Viscosity $\left(\mathrm{cm}^{2} . \mathrm{s}^{-1}\right)$ & $v$ & $2.83 \times 10^{-3}$ & $4.38 \times 10^{-3}$ & $1.8 \times 10^{-3}$ & $2.5 \times 10^{-3}$ & $0.89 \times 10^{-3}$ \\
\hline Thermal Diffusivity $\left(\mathrm{cm}^{2} . \mathrm{s}^{-1}\right)$ & $\alpha$ & 0.4793 & 0.438 & 0.301 & 0.116 & 0.144 \\
\hline Characteristic Velocity $\left(\mathrm{cm}^{-1} \mathrm{~s}^{-1}\right)$ & $u_{o}$ & 0.4793 & 0.438 & 0.301 & 0.116 & 0.144 \\
\hline Eutectic concentration & $\mathrm{C}_{\mathrm{e}}$ & 0.335 & 0.13 & 0.923 & 0.74 & 0.45 \\
\hline Soret Coefficient $\left(\mathrm{K}^{-1}\right)$ & $S_{T}$ & 0.7 & 0.722 & 0.174 & 0.0204 & 0.025 \\
\hline Buoyancy Ratio & $N$ & 1.54 & 9.93 & 0.85 & 3.3 & 1.33 \\
\hline Melt point of pure solvent (K) & $\mathrm{T}_{\mathrm{m}}$ & 504.9 & 504.9 & 504.9 & 504.9 & 544.4 \\
\hline Thermodiffusion Factor & $\alpha_{T}$ & -0.398 & -0.4099 & 0.99 & -0.1753 & -0.26 \\
\hline
\end{tabular}

Figures 5-12 to 5-15 show transient flow at $25 \%$ solidification for Sn-Zn, Sn-Pb, Sn-Cd and $\mathrm{Bi}-\mathrm{Pb}$ binary molten metal. These binary molten metals have $\beta_{C}>\beta_{T}$ or $|N|>1$. The simulation showed that the flow field resulted in a higher concentration variation pattern. Furthermore, when thermodiffusion was included, there was an even higher variation of concentration in the flow pattern. As solidification progressed, the flow became very weak at the boundary between the mushy zone and liquid zone. Finally, there is no flow at the end of 
solidification. The velocity range of the cases without thermodiffusion had a higher value when compared to the simulations with thermodiffusion. For Sn-Zn, Sn-Pb, Sn-Cd, and Bi-Pb cases $D_{T}<0$ (including thermodiffusion), which means that the mass fluxes caused by the Soret effect and normal diffusion have the same direction. Thus, the mass flux had a higher value in the mushy region, which in turn caused a greater temperature.

Figure 5-16 shows the velocity distribution for the Sn-Ga FE simulation. It can be seen that the flow in the mushy region became very weak. The flow field was shifted towards the hot wall of the cavity and weakened in the mushy region. Figure 5-16 also shows that the velocity was higher when thermodiffusion was included. This could be attributed to the fact that $D_{T}$ is greater than 0 . 


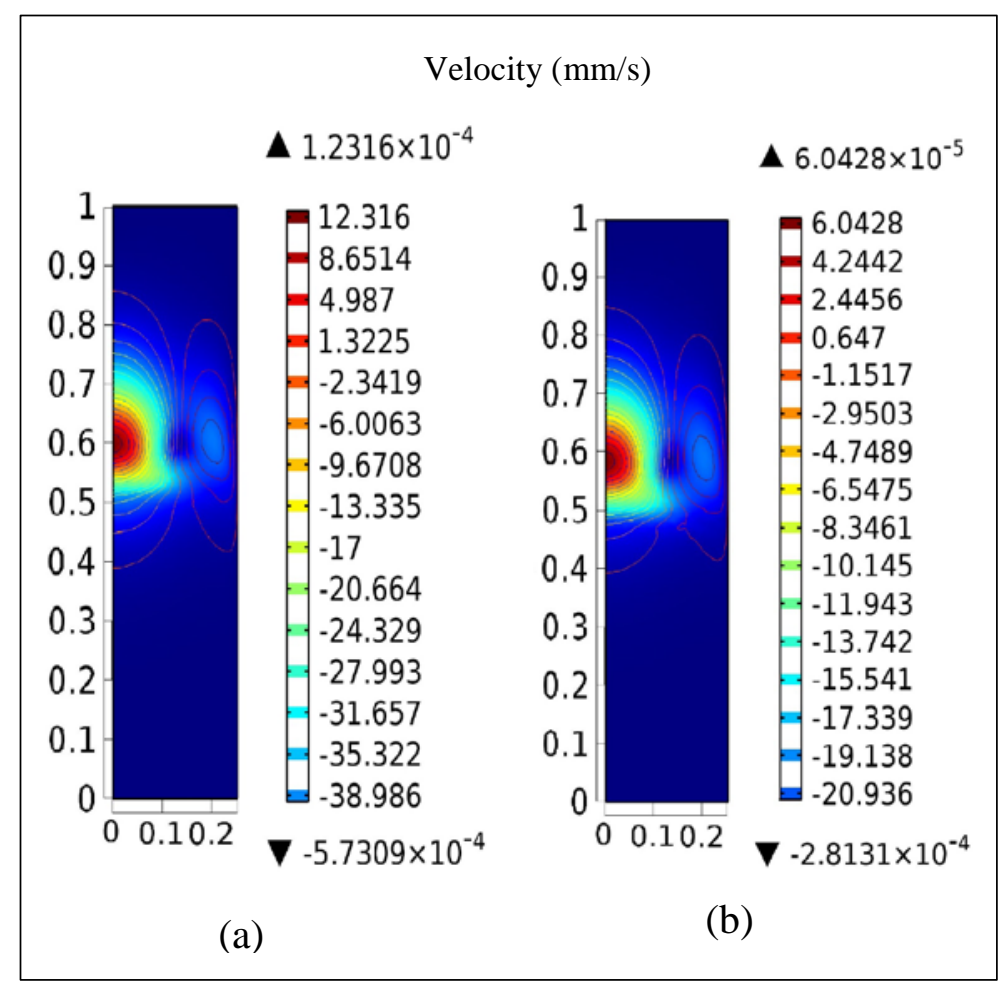

Figure 5 - 12: Simulated result for streamlines at $25 \%$ solidification of Sn-Zn; (a) without thermodiffusion simulation, (b) with thermodiffusion.

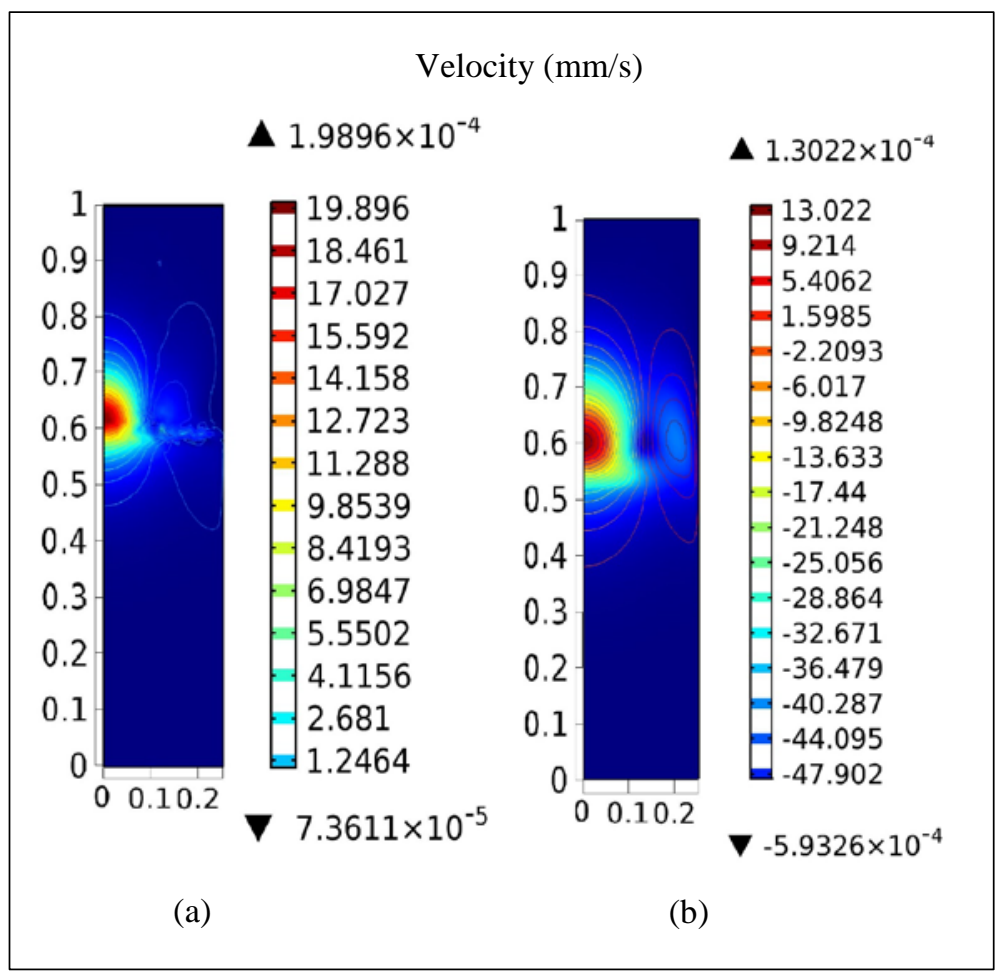

Figure 5 - 13: Simulated result for streamlines at $25 \%$ solidification of Sn-Pb; (a) without thermodiffusion simulation, (b) with thermodiffusion. 


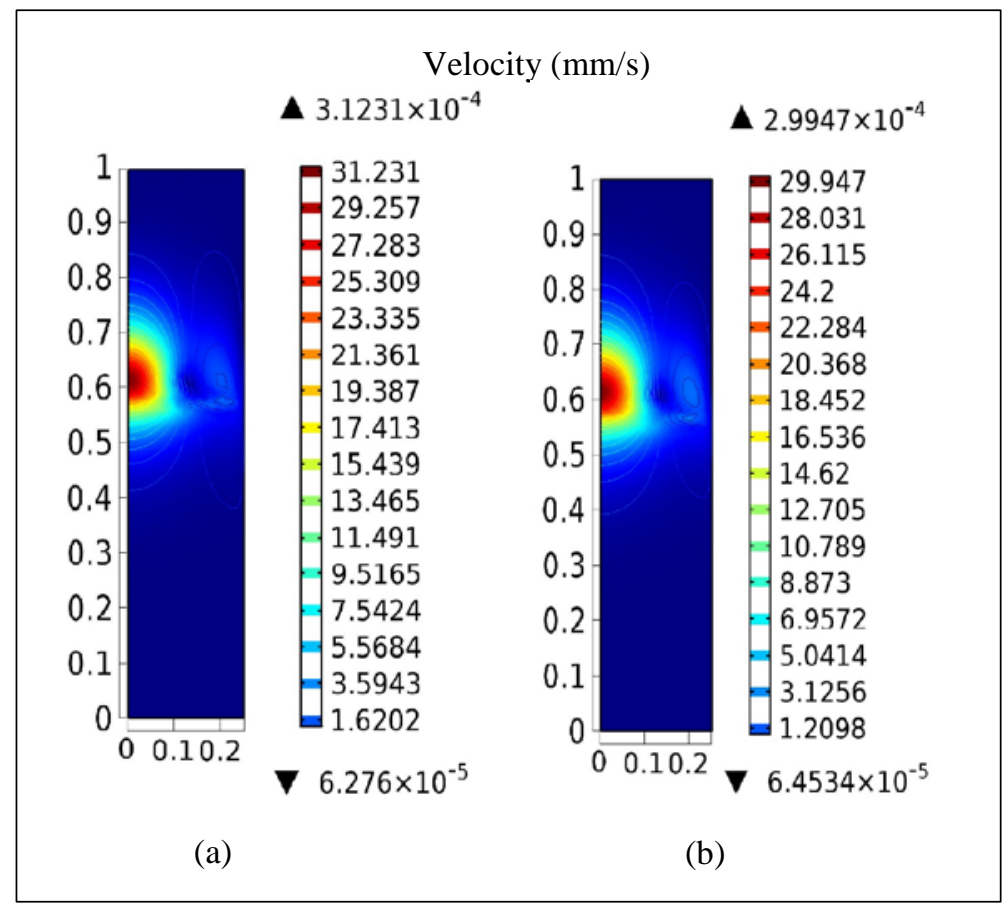

Figure 5 - 14: Simulated result for streamlines at $25 \%$ solidification of Sn-Cd; (a) without thermodiffusion simulation, (b) with thermodiffusion.

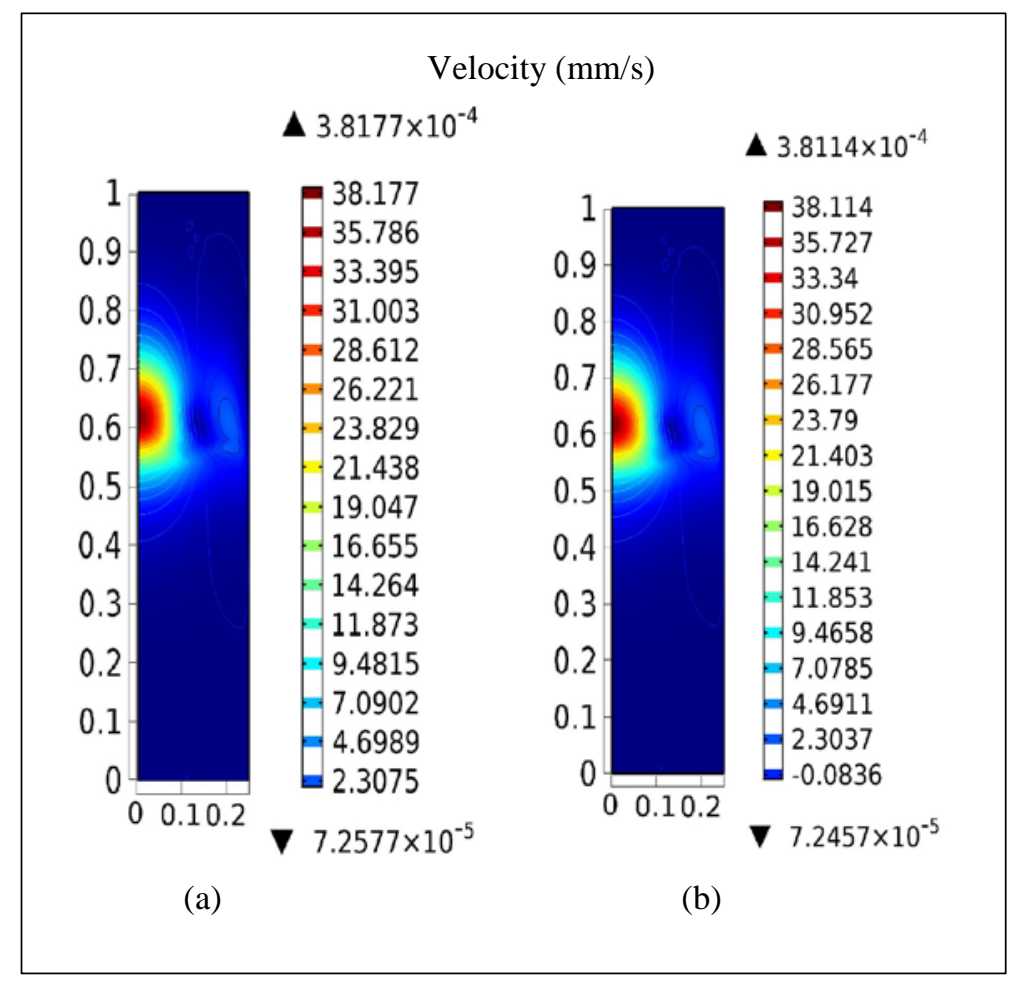

Figure 5 - 15: Simulated result for streamlines at $25 \%$ solidification of $\mathrm{Bi}-\mathrm{Pb}$; (a) without thermodiffusion simulation, (b) with thermodiffusion. 


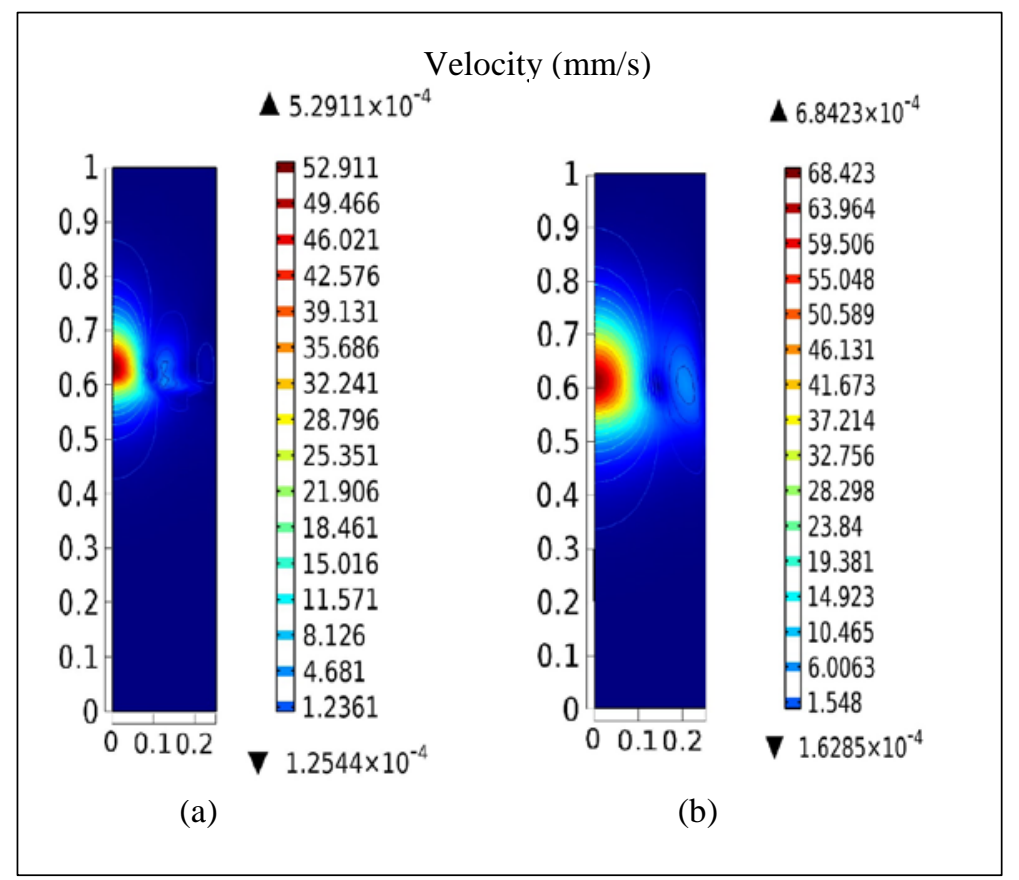

Figure 5 - 16: Simulated result for streamlines at $25 \%$ solidification of Sn-Ga; (a) without thermodiffusion simulation, (b) with thermodiffusion.

Figures 5-17(a) to 5-21(a) show the simulation without thermodiffusion and Figures 517(b) to 5-21(b) show the simulation with the effect of thermodiffusion included. Figures 5-17 to 5-20 depict the temperature distributions for Sn-Cd, Sn-Zn, Sn-Pb, and Bi-Pb whereas Figure 5.21 shows that of Sn-Ga.

In Figures 5-17 to 5-20, the temperature was higher when thermodiffusion was included. This was due to a higher value of mass flux in the mushy region. The temperature distribution across the cavity (lateral direction) was nearly constant in both the solid and liquid zones. On the other hand, in the mushy zone, the temperature varied from the centre of the cavity to the adiabatic wall. 


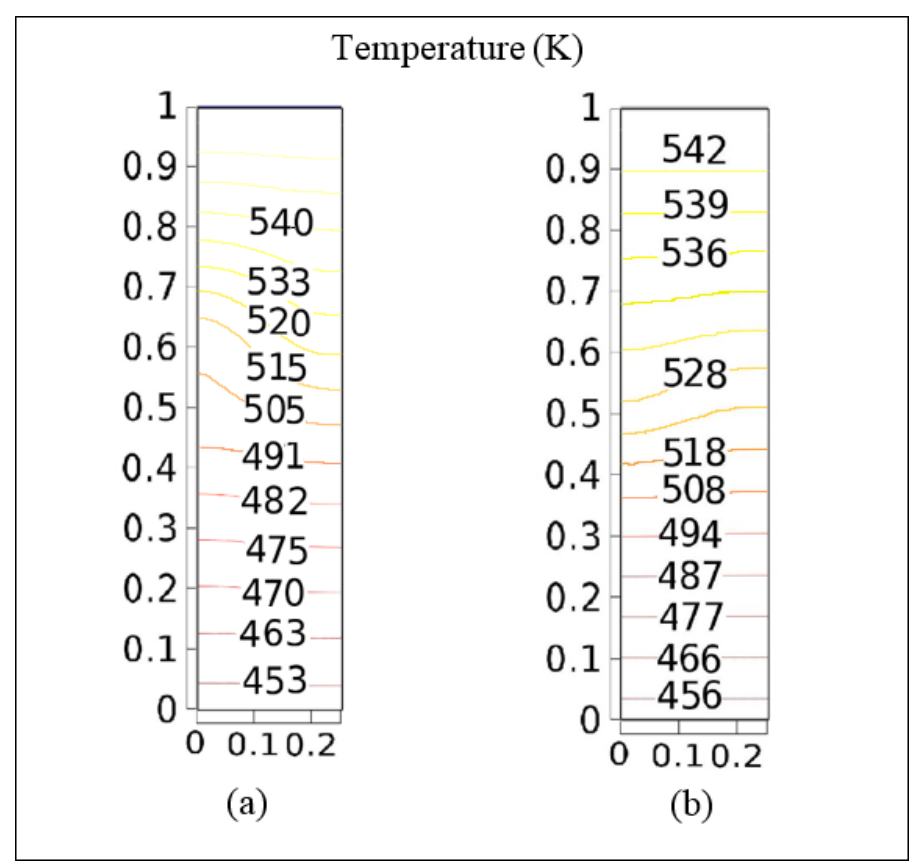

Figure 5 - 17: Simulated result for temperature distribution at $25 \%$ solidification of Sn-Zn; (a) without thermodiffusion simulation, (b) with thermodiffusion.

\begin{tabular}{|c|c|c|c|}
\hline \multicolumn{4}{|c|}{ Temperature $(\mathrm{K})$} \\
\hline 1 & & 1 & \\
\hline 0.9 & 539 & 0.9 & 541 \\
\hline 0.8 & 536 & 0.8 & 537 \\
\hline 0.7 & 533 & 0.7 & 534 \\
\hline 0.6 & 525 & 0.6 & 526 \\
\hline 0.5 & 515 & 0.5 & 516 \\
\hline 0.4 & 505 & 0.4 & $\begin{array}{l}516 \\
507\end{array}$ \\
\hline 0.3 & $\begin{array}{r}491 \\
484\end{array}$ & 0.3 & $\begin{array}{r}492 \\
485\end{array}$ \\
\hline $0.2-$ & 474 & 0.2 & \begin{tabular}{|l|}
485 \\
476 \\
\end{tabular} \\
\hline 0.1 & 463 & 0.1 & 464 \\
\hline 0 & -453 & 0 & 455 \\
\hline 0 & 0.10 .2 & & 0.10 .2 \\
\hline & (a) & & (b) \\
\hline
\end{tabular}

Figure 5 - 18: Simulated result for temperature distribution at $25 \%$ solidification of Sn-Pb; (a) without thermodiffusion simulation, (b) with thermodiffusion. 


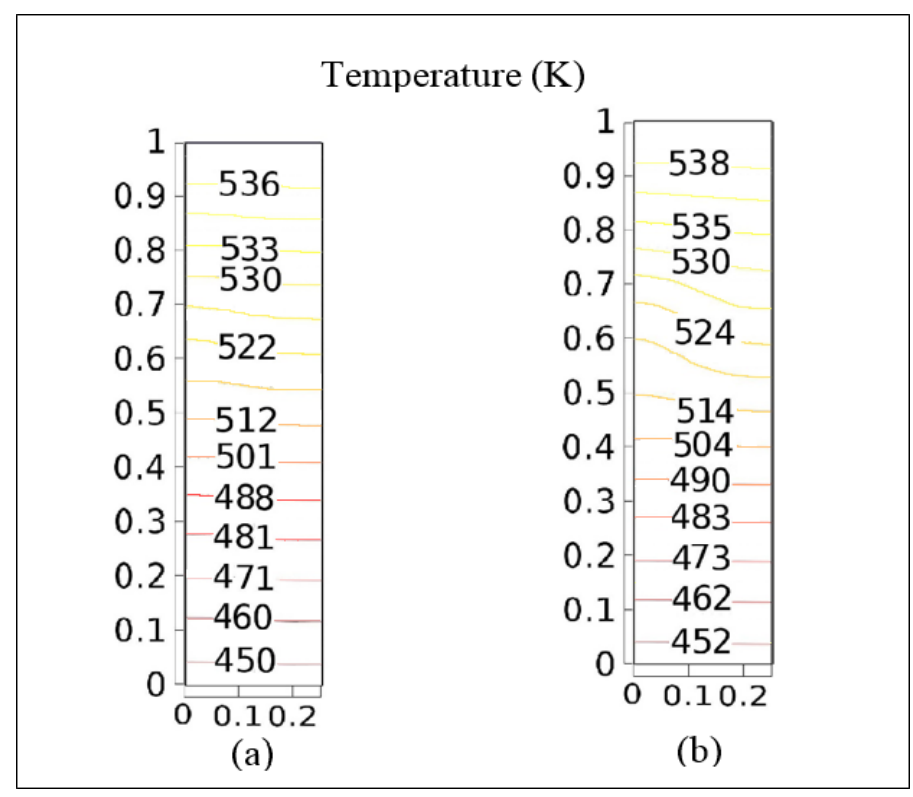

Figure 5 - 19: Simulated result for temperature distribution at $25 \%$ solidification of Sn-Cd; (a) without thermodiffusion simulation, (b) with thermodiffusion.

\begin{tabular}{|c|c|c|c|}
\hline \multicolumn{4}{|c|}{ Temperature (K) } \\
\hline \multicolumn{2}{|l|}{1} & \multicolumn{2}{|l|}{1} \\
\hline 0.9 & 540 & 0.9 & 535 \\
\hline 0.8 & 240 & 0.8 & 532 \\
\hline 0.7 & 530 & 0.7 & 529 \\
\hline 0.6 & 520 & 0.6 & 521 \\
\hline 0.5 & -509 & 0.5 & \\
\hline 0.4 & -493 & 0.4 & $\begin{array}{l}511 \\
501\end{array}$ \\
\hline 0.3 & $\begin{array}{r}-485 \\
-479\end{array}$ & 0.3 & 487 \\
\hline 0.2 & $\begin{array}{r}4 / 9 \\
-464 \\
\end{array}$ & 0.2 & $\begin{array}{l}480 \\
470\end{array}$ \\
\hline 0.1 & -455 & 0.1 & 459 \\
\hline 0 & -445 & 0 & 449 \\
\hline & 0.10 .2 & & 0.10 .2 \\
\hline & (a) & & (b) \\
\hline
\end{tabular}

Figure 5 - 20: Simulated result for temperature distribution at $25 \%$ solidification of Bi-Pb; (a) without thermodiffusion simulation, (b) with thermodiffusion.

Figure 5-21 shows the temperature distribution of Sn-Ga. On average, the temperature decreased when the thermodiffusion effect was included, which was opposite to the earlier four 
cases. This was attributed to $D_{T}$ being greater than 0 . A positive $D_{T}$ caused an increase in the solidification growth rate and a higher solid fraction. Figure 5-22 shows the progress of solidification for all of the binary alloy systems (Sn-Zn, Sn-Pb, Sn-Cd, Bi-Pb, and Sn-Ga) including thermodiffusion. The progress of solidification was similar for four of the binary molten metals (Sn-Zn, Sn-Pb, Sn-Cd, and Bi-Pb), but slightly differed for the Sn-Ga system.

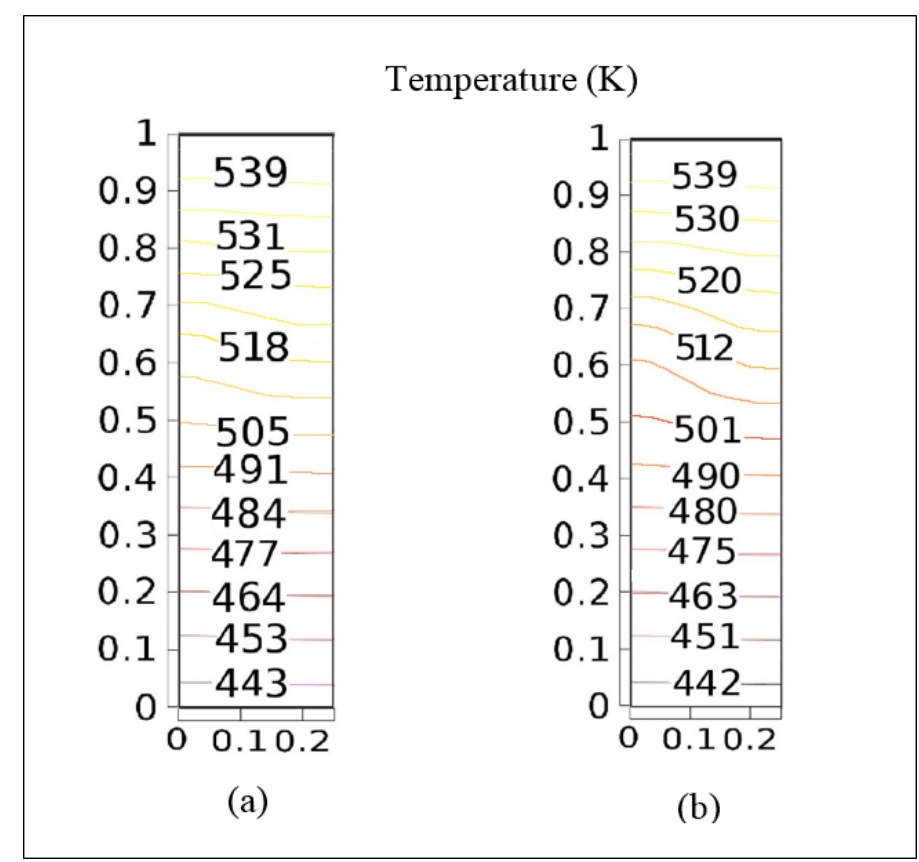

Figure 5 - 21: Simulated result for temperature distribution at $25 \%$ solidification of Sn-Ga; (a) without thermodiffusion simulation, (b) with thermodiffusion. 


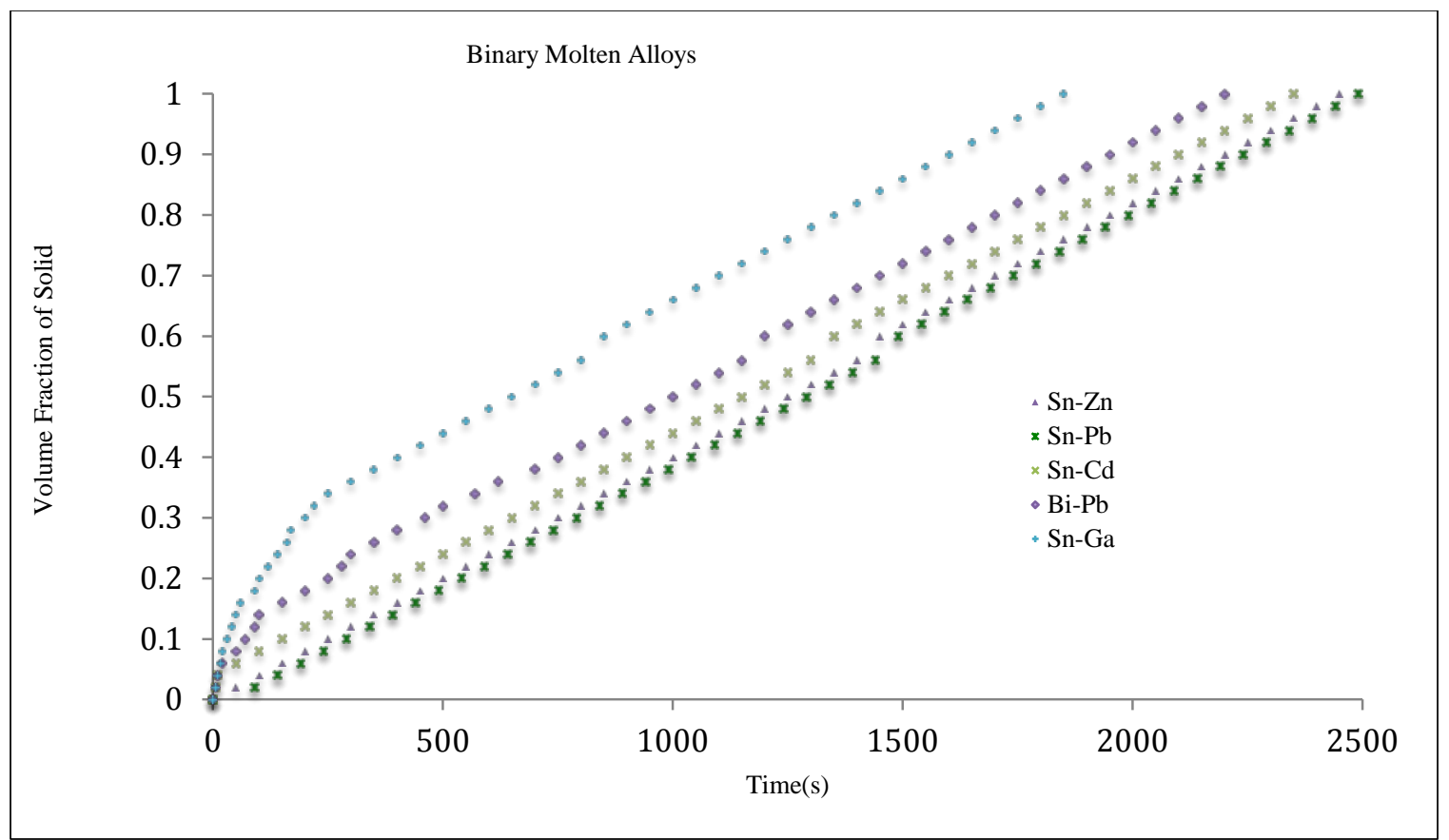

Figure 5 - 22: Progress of solidification for binary molten metals; Sn-Zn, Sn-Pb, Sn-Cd, Bi-Pb, and SnGa.

Figures 5-23 to 5-27 show the comparison of the average concentration variation along the height of the cavity for the cases with and without thermodiffusion included. This comparison was carried out for the aforementioned five binary molten metals. The average composition variation at the end of solidification for the five cases showed that the thermodiffusion had only a small effect on concentration variation. For the binary molten metals of Sn-Zn, Sn-Pb, Sn-Cd, and Bi-Pb, Figures 5-23 to 5-26, including thermodiffusion in the FE simulations increased the variation in concentrations. This was attributed to the fact that $D_{T}$ was less than zero. Conversely, for the case of Sn-Ga, Figure 5-27, the thermodiffusion decreased the variation in concentration. 


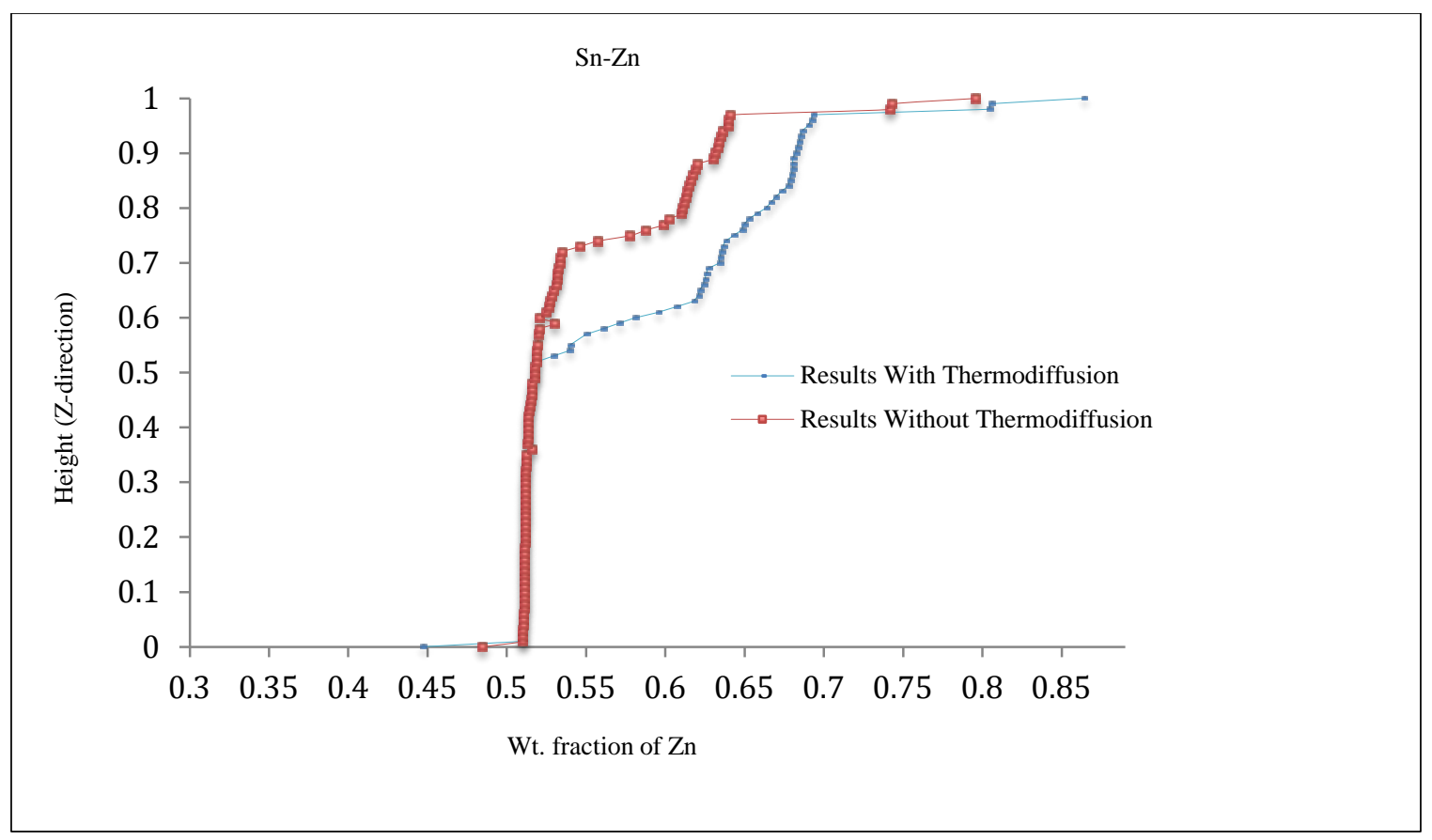

Figure 5 - 23: Simulated result for composition distribution at $25 \%$ solidification of Sn-Zn; (a) without thermodiffusion simulation, (b) with thermodiffusion.

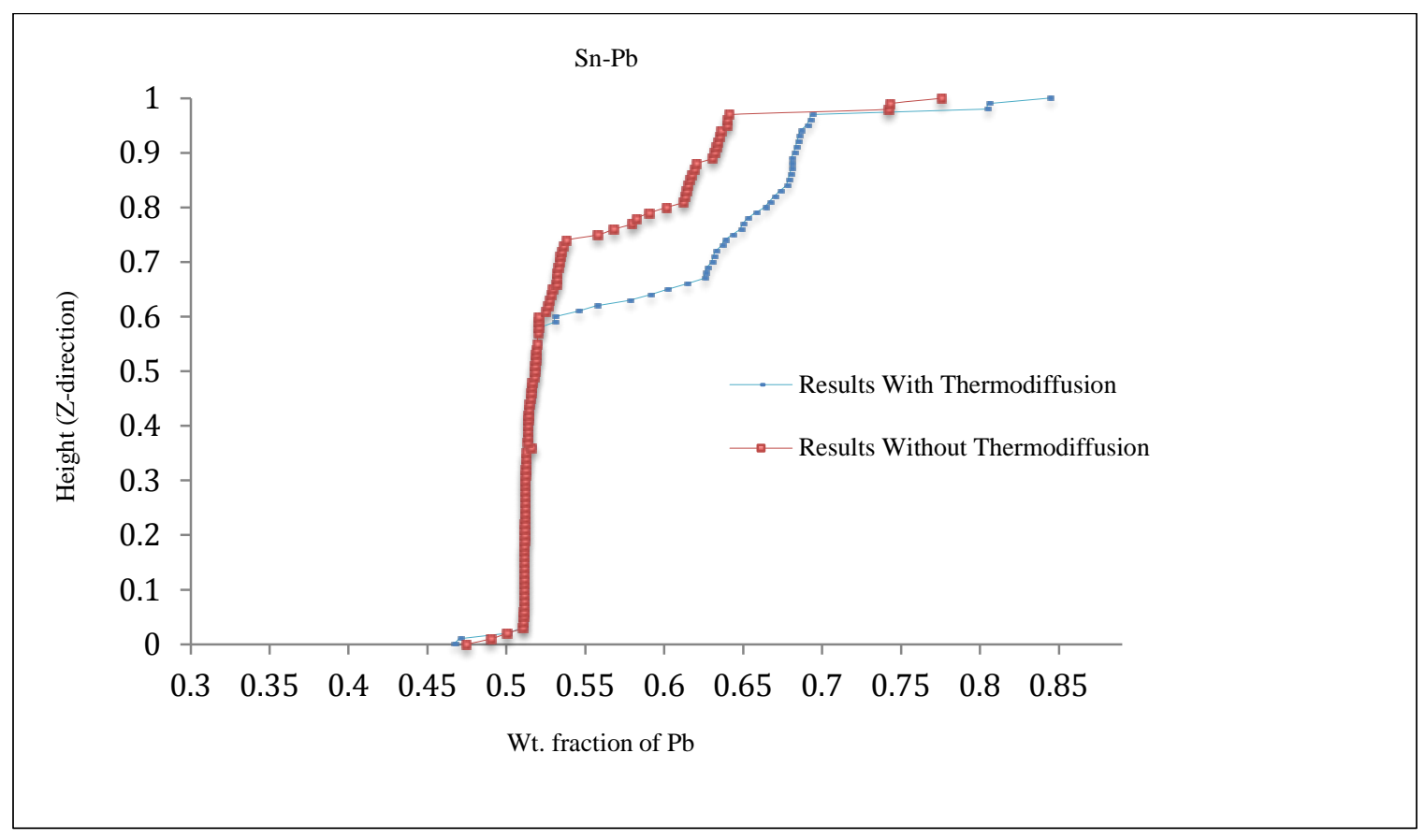

Figure 5 - 24: Simulated result for composition distribution at 25 \% solidification of Sn-Pb; (a) without thermodiffusion simulation, (b) with thermodiffusion. 


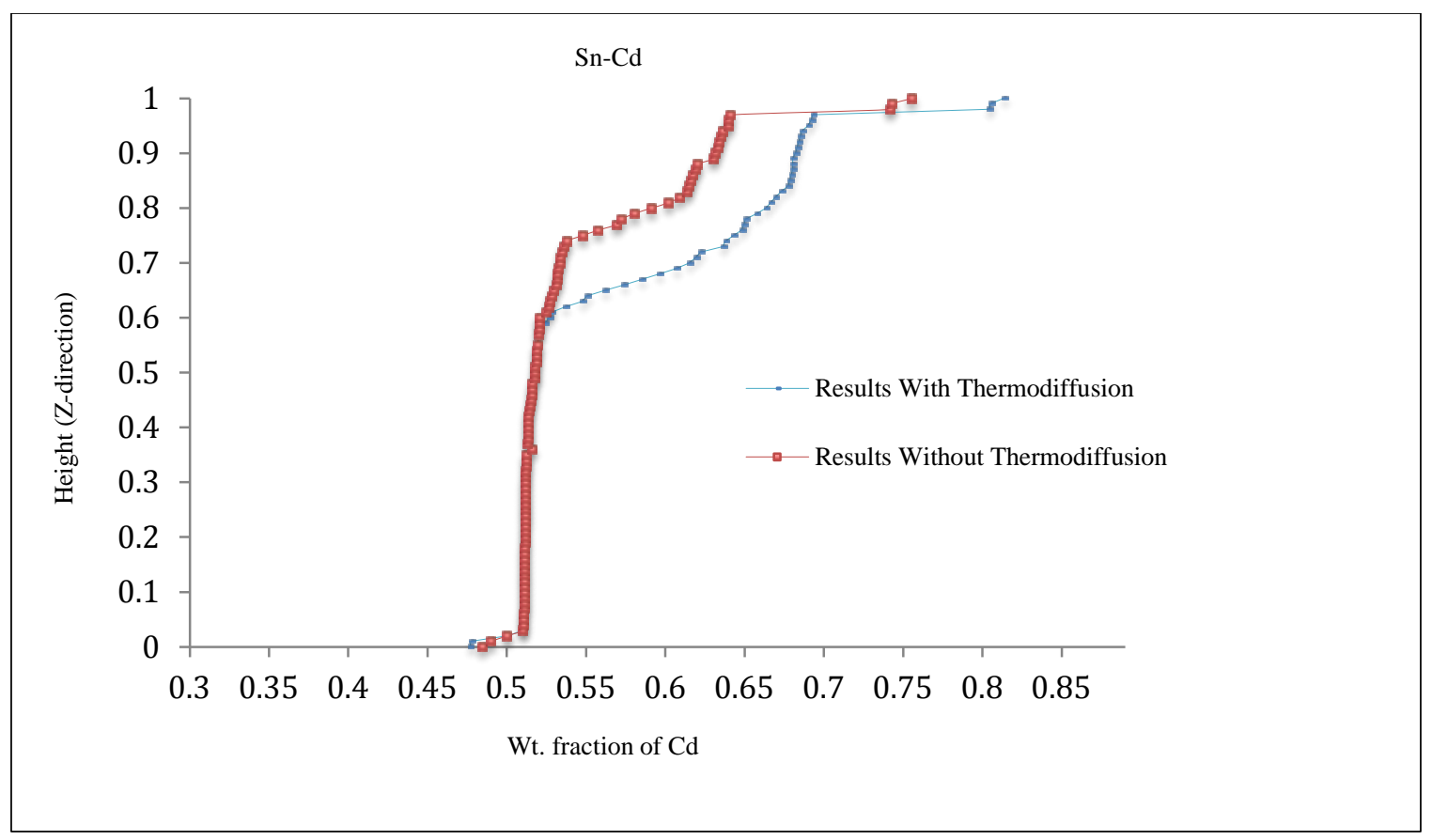

Figure 5 - 25: Simulated result for composition distribution at $25 \%$ solidification of Sn-Cd; (a) without thermodiffusion simulation, (b) with thermodiffusion.

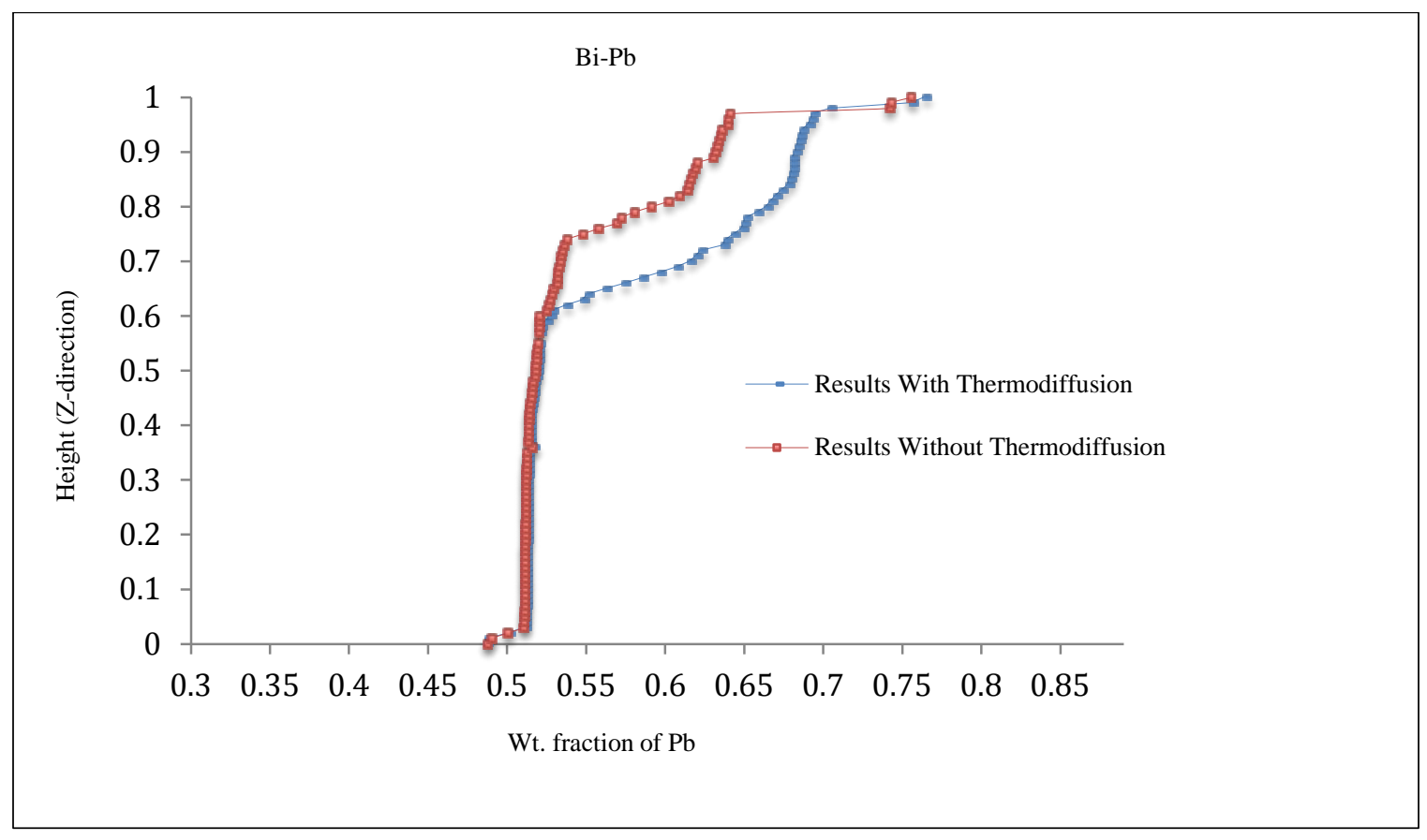

Figure 5 - 26: Simulated result for composition distribution at $25 \%$ solidification of Bi-Pb; (a) without thermodiffusion simulation, (b) with thermodiffusion. 


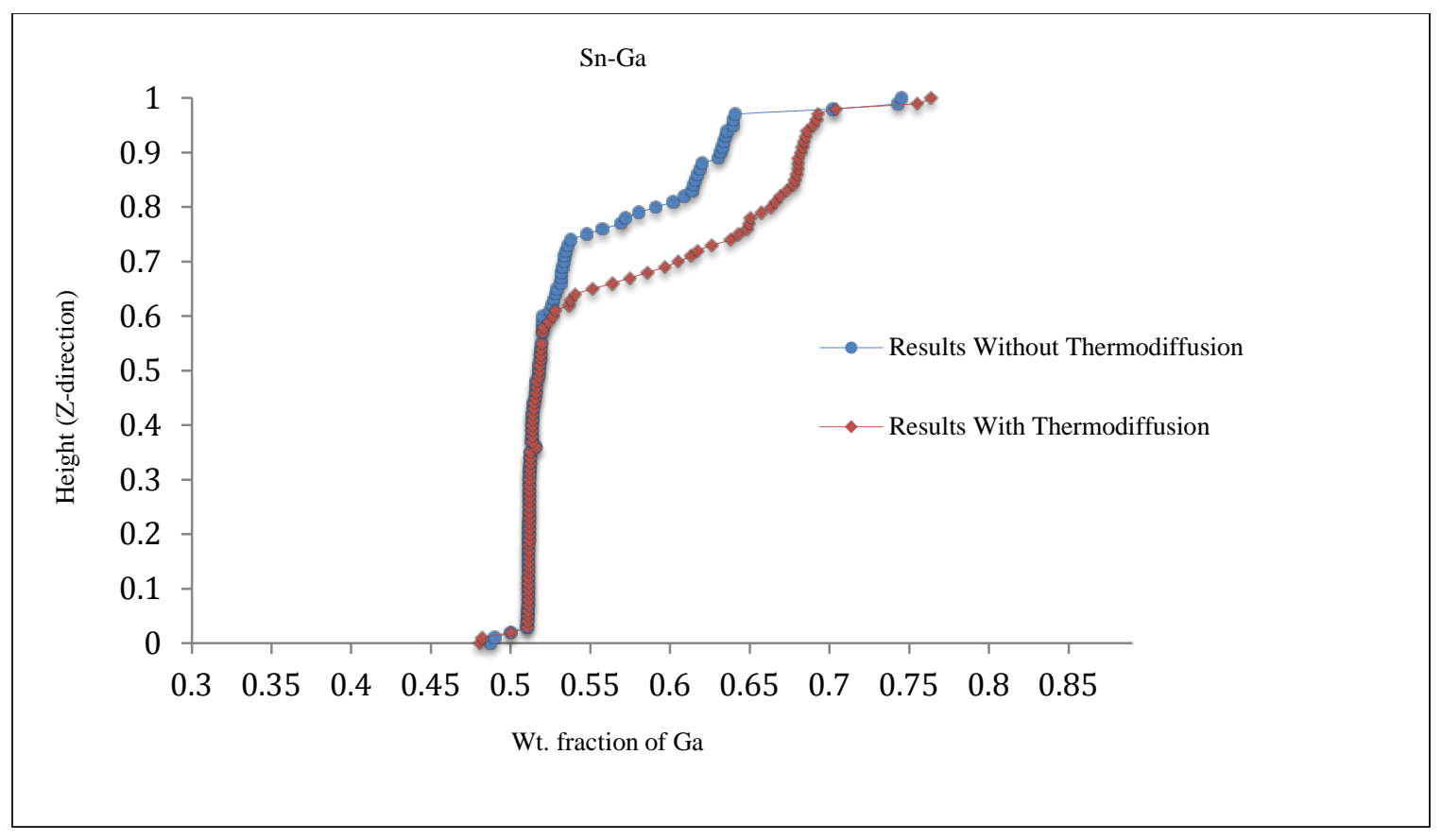

Figure 5 - 27: Simulated result for composition distribution at $25 \%$ solidification of Sn-Ga; (a) without thermodiffusion simulation, (b) with thermodiffusion.

\subsubsection{Discussion of Results}

The effect of the material properties on thermosolutal convection and consequently on the concentration variation is clearly evident from the comparative study of the aforementioned binary molten metals. The simulations for the solidification of Sn-Zn, Sn-Pb, Sn-Cd, and Bi-Pb binary molten metals were carried out. These binary molten metals have $\beta_{C}>\beta_{T}$ or $|N|>1$. As such, the concentration force played a significant role in convection. The concentration force assisted the thermal force in causing a disturbance to the flow and making it unstable. This flow field resulted in a higher concentration variation pattern. Moreover, when thermodiffusion was included, the $D_{T}$ became negative, which resulted in a higher variation of concentration in the flow pattern. 
Velocity streamlines were shown by a weak upward flow near the centre of the cavity. The velocity range in the cases without thermodiffusion had higher values compared to the simulations with thermodiffusion. Positive or negative values of $D_{T}$ increased or decreased the solidified fraction in the mushy region, respectively.

In the cases that $D_{T}>0$, thermal and solutal fluxes acted in opposite directions, and, as a consequence, thermodiffusion assisted the growth of the solid fraction. The flow in the mushy region became very weak. As solidification progressed, the flow field gradually shifted towards the hot wall of the cavity and weakened in strength due to the spread of the mushy region in the cavity. Macrosegregation gradually built up with the progress of solidification. As for the macrosegregation, it was seen that there was depletion of solute along the right wall and deposition of solute along the middle of the cavity. The downward thermal force near the mushy zone took the solute with it and deposited it near the middle of the cavity [102]. The dominance of the thermal force over the concentration force for Sn-Ga binary molten metal was observed, which was due to $|N|<1$. The fluid was well mixed in the cavity except in the mushy zone.

Table 5-3 tabulates the percent variation in all five cases. The minimum and maximum variations in concentration were calculated using Eq. 5.17.

Table 5 - 3: Variation of concentration for five binary molten metals for with and without thermodiffusion effect

\begin{tabular}{|c|c|c|c|c|c|c|c|}
\hline \multirow[t]{2}{*}{ Alloy } & \multirow{2}{*}{$\begin{array}{l}\text { Initial } \\
\text { concentration }\end{array}$} & \multicolumn{2}{|c|}{$\begin{array}{l}\text { Without } \\
\text { Thermodiffusion }\end{array}$} & \multicolumn{2}{|c|}{$\begin{array}{l}\text { With } \\
\text { Thermodiffusion }\end{array}$} & \multirow{2}{*}{$\begin{array}{l}\text { Min } \\
\text { Variation }\end{array}$} & \multirow{2}{*}{$\begin{array}{l}\text { Max } \\
\text { Variation }\end{array}$} \\
\hline & & Min & Max & Min & $\max$ & & \\
\hline Sn-Zn & 0.5 & 0.4845 & 0.7955 & 0.4465 & 0.8635 & $-7 \%$ & $8 \%$ \\
\hline $\mathrm{Sn}-\mathrm{Pb}$ & 0.5 & 0.4745 & 0.7755 & 0.4665 & 0.8435 & $-1 \%$ & $8 \%$ \\
\hline Sn-Cd & 0.5 & 0.4845 & 0.7555 & 0.4765 & 0.8135 & $-1 \%$ & $7 \%$ \\
\hline $\mathrm{Bi}-\mathrm{Pb}$ & 0.5 & 0.4875 & 0.7555 & 0.4865 & 0.7635 & $-3 \%$ & $1 \%$ \\
\hline Sn-Ga & 0.5 & 0.4805 & 0.7635 & 0.4875 & 0.7455 & $1 \%$ & $-2 \%$ \\
\hline
\end{tabular}


Table 5-3 shows that the concentration variation was small in the case of Sn-Ga. The reason is partly due to its $D_{T}$ being greater than zero, and its $N$ being smaller than one. Note that $N$ is the ratio of solutal buoyancy to thermal buoyancy. Having small solutal buoyancy gives relatively smaller Raleigh numbers and high resistance to flow in the mushy zone [142]. On the other hand, in the cases of Sn-Zn, Sn-Pb, Sn-Cd, and Bi-Pb concentration variations were higher. The variation in concentration is directly related to the value of $N$ : the higher the value of $N$, the higher the value of the concentration variation [139].

Thus, it is seen that the material properties affect the thermosolutal convection and consequently the concentration variation in a very significant way. The role of the buoyancy ratio $(N)$, both its magnitude and sign, during the evolution of the composition change was demonstrated through a comparative study.

\subsection{Summary}

The finite element method was used to construct a 2D axisymmetric model of 3D cylindrical cavity. The finite element model was validated against existing published research. Subsequently, appropriate boundary conditions were implemented for Sn-Bi, Sn-Cd, Sn-Zn, Sn$\mathrm{Pb}$, Sn-Ga, and Bi-Pb binary molten metal mixtures. The finite element simulation revealed that for positive thermodiffusion $\left(\mathrm{D}_{\mathrm{T}}>0\right.$ and $\left.\mathrm{N}<1\right)$, the presence of thermodiffusion results in a uniform distribution of concentrations during the solidification process. On the other hand, when thermodiffusion is negative $\left(\mathrm{D}_{\mathrm{T}}<0\right.$ and $\left.\mathrm{N}>1\right)$, the quality of the solidified alloy is poor as compared to the case when $\mathrm{D}_{\mathrm{T}}$ is positive and consequently results in a more non-uniform concentration distribution. 


\section{CHAPTER SIX}

\section{Summary, Conclusion, and Future Work}

\subsection{Summary}

This research work was performed primarily to study the solidification of binary molten metals in the presence of thermodiffusion. This work was carried out in three steps in chronological order.

First, it was proposed an improved expression for the thermodiffusion factor, which was derived based on the models of Eslamian and Maier. The thermodiffusion factors of Sn-Bi, Sn$\mathrm{Zn}, \mathrm{Sn}-\mathrm{Pb}, \mathrm{Sn}-\mathrm{Cd}, \mathrm{Sn}-\mathrm{Ga}$, and $\mathrm{Bi}-\mathrm{Pb}$ were estimated using the proposed expression. The numerical values were compared to experimental data and many well-known research data. It was shown that the proposed expression gave numerical values with greater accuracy when compared to other proposed expressions and when compared to experimental data. The numerical and experimental values are summarized in Tables 3-4 and 3-5.

In the second step, the proposed thermodiffusion factors were implemented into the finite element (FE) model of Sn-Bi binary molten metal, and it was also included external natural convection and surface radiation to ambient. The effects of natural convection and surface radiation and thermodiffusion were included in FE simulation to examine their effect on velocity, temperature, and concentration distribution of Sn-Bi molten metal alloy in a rectangular quartz cavity. It was determined that the external natural convection and surface radiation to ambient has no significant effect and could be omitted.

In the third step, it was focused on the main purpose of this research work, which was 
simulation of the process of solidification of the aforementioned binary molten metals. The author constructed an FE model for a cylindrical cavity and validated the model by reproducing an accepted published result. Next, the effect of external natural convection and surface radiation to ambient were determined negligible thus, the author did not include these phenomena in the FE simulations, and their attention was focused primarily on the effect of thermodiffusion. It was determined that in solidification processes, the effect of thermodiffusion needs to be included as its effect is notable on the concentration distribution.

\subsection{Conclusion}

Here, finite element method was used to simulate the solidification process of six binary molten metals and the following were determined:

- A model was proposed to predict the thermodiffusion factor for binary molten metal alloys. The proposed model was based on the assumption that there are three forces acting on ions: A thermal gradient, an induced concentrated gradient, and an induced electric field. The proposed model is within the framework of linear non-equilibrium thermodynamics and considers the thermodiffusion factor in terms of the net heats of transport of the electronic and ion-ion thermal interactions.

- Numerical simulations were carried out using the proposed thermodiffusion factor to investigate the effect of surface radiation and external natural convection in a rectangular quartz cavity filled with Sn-Bi. Here two specific cases of bottom heating and top heating were studied. In both cases, the surface radiation and external natural convection had negligible effects on velocity, temperature, and concentration distributions. 
- It was concluded that bottom heating promotes the convective flow that in turn causes the temperatures and concentrations to become dependent on the Ra number. With an Ra number between 1000 and 2000, convective flows start to develop in the cell.

- Thermodiffusion produces a separating effect in binary mixtures in the top heating case, whereas in the bottom heating case, thermodiffusion is only effective in low Ra number conditions. With large Ra numbers, natural convection is present and dominant, while thermodiffusion becomes a secondary effect.

- At first, numerical simulations based on the governing equations were used to examine the behaviour of Sn-Bi molten metal during vertical solidification in a 2D-axisymmetric FE model of a cylindrical cavity. The FE model was validated and the formation of flow field and its effect on temperature distribution, fraction of the liquid phase, and concentration distribution were predicted for two cases of with and without thermodiffusion included.

- Thermodiffusion modifies the mass transfer and has a slight effect on the temperature and velocity distributions. However, the velocities in both cases are very small and generally are not considered.

- The solid phase fraction in a mushy zone as well as the height of the mushy zone in vertical direction increased as the result of including thermodiffusion in the simulations. In the case without the effect of thermodiffusion, there was a higher tendency for variation in average composition along the vertical direction.

- The solidification of five additional binary molten metals in the presence and absence of thermodiffusion were examined. The effect of material properties on thermosolutal convection and consequently on the concentration variation is clearly evident from the 
comparative study that was carried out on the five aforementioned binary molten metals. The following conclusions are drawn from this work:

- For Sn-Zn, Sn-Pb, Sn-Cd, and Bi-Pb, $D_{T}$ is negative, thus the molecular diffusion and thermal diffusion have the same direction. More specifically, the thermal force assists the concentration force, and the flow field is unstable.

- In Sn-Ga and Sn-Bi binary molten metals, the thermal force opposes the concentration force, and the flow is more stable. Additionally, the variation of concentration is lower in these systems than in the other systems.

- In the cases of $\mathrm{Sn}-\mathrm{Ga}$ and $\mathrm{Sn}-\mathrm{Bi}$, the range of composition variation along the cavity in the presence of thermodiffusion is less than the case without thermodiffusion.

- In contrast, the composition variation for $\mathrm{Sn}-\mathrm{Zn}, \mathrm{Sn}-\mathrm{Pb}, \mathrm{Sn}-\mathrm{Cd}$, and $\mathrm{Bi}-\mathrm{Pb}$ in the presence of thermodiffusion is increased. The concentration variation increases as the ratio of solutal buoyancy to thermal buoyancy $(N)$ increases. It was demonstrated that during the solidification of Sn-Zn, Sn-Pb, Sn-Cd, and Bi-Pb, the solutal force has a significant role in thermosolutal convection.

- There are few other factors that could potentially influence the concentration variation such density or melting point. Here, it was concluded that the effect of thermodiffusion is more pronounced in solidification, and it has provided a more realistic picture of the flow and of the composition patterns. Thus, it is undesirable to invoke such simplification in solidification by neglecting thermodiffusion. 


\subsection{Future Work}

The preceding presented here has outlined the effect of thermodiffusion as well as the effect of natural convection and surface radiation for solidification of binary molten metals in a cylindrical cavity. Future work could involve:

- Experimental evaluation of solidification of binary molten metals studied numerically.

- Finite element simulation to evaluate the solidification process of ternary molten metals.

- Application of current work in manufacturing of solar panels

- The current research results could further be used in material processing industry 


\section{APPENDIX A}

\section{Non-Dimensionalization}

\section{A.1 Introduction}

As described in chapter 4, the dimensionless forms of the governing equations are more suitable for the computing purposes where and when the time is of the essence.

Frank White [187] defined the dimensional analysis as: a method for decreasing the number and complexity of experimental variables that influences a given physical phenomenon, by applying a kind of compacting technique.” The author, also, expressed the Principle of Dimensional Homogeneity (PDH) as: If an equation truly states an appropriate relationship between variables in physical procedure, it will be dimensionally homogenous; that is, each of its additive terms will have the same dimensions. Dimensionless parameters have many advantages, some of them are: less number of variables, saving time and money by reducing the number of experiments, simulations and/or communications. Increasing the fundamental variables regards to typical values and forming dimensionless parameters offers an amount of the relative importance of the several terms in the equations and identifies the governing physical phenomena [187].

The dimensionless parameters used to change equations (3.1) to (3.14) to the dimensionless form are illustrated appendix (B). In the non-dimensional examination, some other parameters develop, such as the Reynolds number $R e$, the Prandtl number $P r$, and Schmidt number Sc. The non-dimensional equations for fluid layer are: 


\section{A.2 Dimensionless Form of Governing Equations}

The governing equations are rendered dimensionless by using the following non-dimensional variables :

$$
\begin{gathered}
R e=\sqrt{G r} \\
\operatorname{Pr}=\frac{C_{p} \mu}{k} \\
G r_{c}=\frac{g \beta_{c} \Delta C L^{3}}{\vartheta^{2}} \\
G r_{T}=\frac{g \beta_{T} \Delta C L^{3}}{\vartheta^{2}} \\
u_{0}=\sqrt{g \beta_{T} \cdot \Delta T \cdot L} \\
N=\frac{\beta_{C}}{\beta_{T}} \\
D_{m}=\frac{1}{R e \cdot S c}
\end{gathered}
$$

The detailed and step-by-step non-dimensionalization of all the governing equations is discussed as follow.

The continuity equation of fluid dynamics is a mathematical form which states that mass is always conserved in fluid systems regardless the complexity or direction of flow.

$\frac{\partial u}{\partial x}+\frac{\partial v}{\partial y}+\frac{\partial w}{\partial z}=0$

$\frac{\partial U}{\partial X}+\frac{\partial V}{\partial Y}+\frac{\partial W}{\partial Z}=0$ 
Navier-Stokes equations can be used to represent the momentum balance equations for the flow in the fluid layer. The momentum balance equation in the $\mathrm{X}$ - direction can be expressed as follows:

$\rho\left[\frac{\partial u}{\partial t}+u \frac{\partial u}{\partial x}+v \frac{\partial u}{\partial y}+w \frac{\partial u}{\partial z}\right]=-\frac{\partial p}{\partial x}+\mu\left[\frac{\partial^{2} u}{\partial x^{2}}+\frac{\partial^{2} u}{\partial y^{2}}+\frac{\partial^{2} u}{\partial z^{2}}\right]$

Substitute the dimensional variables and then get:

$$
\begin{gathered}
\rho_{f}\left[\frac{\partial\left(U \cdot u_{0}\right)}{\partial\left(\frac{t \cdot L}{u_{0}}\right)}+\left(U \cdot u_{0}\right) \frac{\partial\left(U \cdot u_{0}\right)}{\partial(X \cdot L)}+\left(V \cdot u_{0}\right) \frac{\partial\left(U \cdot u_{0}\right)}{\partial(Y \cdot L)}+\left(W \cdot u_{0}\right) \frac{\partial\left(U \cdot u_{0}\right)}{\partial(Z \cdot L)}\right. \\
\left.=-\frac{\partial\left(\frac{P \cdot \mu \cdot u_{0}}{L}\right)}{\partial(X \cdot L)}+\mu\left[\frac{\partial}{\partial(X \cdot L)} \cdot \frac{\partial\left(U \cdot u_{0}\right)}{\partial(X \cdot L)}+\frac{\partial}{\partial(Y \cdot L)} \cdot \frac{\partial\left(U \cdot u_{0}\right)}{\partial(Y \cdot L)}+\frac{\partial}{\partial(Z \cdot L)} \cdot \frac{\partial\left(U \cdot u_{0}\right)}{\partial(Z \cdot L)}\right]\right] \\
\rho_{f} \frac{u_{0}^{2}}{L}\left[\frac{\partial U}{\partial t}+U \frac{\partial U}{\partial X}+V \frac{\partial U}{\partial Y}+W \frac{\partial U}{\partial Z}\right]=-\mu u_{0} \frac{\partial P}{\partial X}+\mu \frac{u_{0}}{L^{2}}\left[\frac{\partial^{2} U}{\partial X^{2}}+\frac{\partial^{2} U}{\partial Y^{2}}+\frac{\partial^{2} U}{\partial Z^{2}}\right]
\end{gathered}
$$

Multiply by $\frac{L^{2}}{\mu \cdot u_{0}}$ :

and then get the non-dimension form of mentioned equation :

$R e\left[\frac{\partial U}{\partial t}+U \frac{\partial U}{\partial X}+V \frac{\partial U}{\partial Y}+W \frac{\partial U}{\partial Z}\right]=-\frac{\partial P}{\partial X}+\left[\frac{\partial^{2} U}{\partial X^{2}}+\frac{\partial^{2} U}{\partial Y^{2}}\right]$

Momentum equation in Y-Direction: 
In the $\mathrm{Y}$ - direction, and because there is a buoyancy-driven flow induced by temperature differences and should be considered in computation, the momentum balance equation includes the Boussinesq approximation. The Boussinesq approximation states that the difference of a fluid density is too small and can be ignored except when it multiplied by the term of gravity acceleration (g), and then the difference can be considered. The Boussinesq approximation can be expressed as:

$\rho_{f}=\rho_{o .} g\left[\beta_{T}(T-T o)-\beta_{C}(C-C o)\right]$

From equation (A.7), it can be noted that the fluid density is a linear function of both, fluid temperature and concentration. The momentum balance equation in the $\mathrm{Y}$ - direction can be expressed as follows:

$$
\rho_{f}\left[\frac{\partial v}{\partial t}+u \frac{\partial v}{\partial x}+v \frac{\partial v}{\partial y}+w \frac{\partial v}{\partial z}\right]=-\frac{\partial p}{\partial y}+\mu\left[\frac{\partial^{2} v}{\partial x^{2}}+\frac{\partial^{2} v}{\partial y^{2}}+\frac{\partial^{2} v}{\partial z^{2}}\right]-\rho_{f} \cdot g\left[\beta_{T}\left(T-T_{0}\right)-\beta_{C}\left(C-C_{0}\right)\right]
$$

Substitute the dimensional variables and then get:

$$
\begin{aligned}
& \rho_{f}\left[\frac{\partial\left(V \cdot u_{0}\right)}{\partial\left(\frac{t \cdot L}{u_{0}}\right)}+\left(U \cdot u_{0}\right) \frac{\partial\left(V \cdot u_{0}\right)}{\partial(X \cdot L)}+\left(V \cdot u_{0}\right) \frac{\left(\partial V \cdot u_{0}\right)}{\partial(Y \cdot L)}+\left(W \cdot u_{0}\right) \frac{\partial\left(V \cdot u_{0}\right)}{\partial(Z \cdot L)}\right]=-\frac{\partial\left(\frac{P \cdot \mu \cdot u_{0}}{L}\right)}{\partial(Y \cdot L)}+\mu\left[\frac{\partial}{\partial(X \cdot L)} \cdot \frac{\partial\left(V \cdot u_{0}\right)}{\partial(X \cdot L)}\right. \\
& \left.+\frac{\partial}{\partial(Y \cdot L)} \cdot \frac{\partial\left(V \cdot u_{0}\right)}{\partial(Y \cdot L)}+\frac{\partial}{\partial(Z \cdot L)} \cdot \frac{\partial\left(V \cdot u_{0}\right.}{\partial(Y \cdot L)}\right]-\rho_{f} \cdot g\left[\beta_{T}\left(T-T_{0}\right)-\beta_{C}\left(C-C_{0}\right)\right]
\end{aligned}
$$




$$
\begin{aligned}
\rho_{f} \cdot \frac{u_{0}^{2}}{L}\left[\frac{\partial V}{\partial t}+\right. & \left.U \frac{\partial V}{\partial X}+V \frac{\partial V}{\partial Y}+Z \frac{\partial V}{\partial Z}\right] \\
& =-\mu \cdot \frac{u_{0}}{L^{2}} \cdot \frac{\partial P}{\partial Y}+\frac{\mu \cdot u_{0}}{L}\left[\frac{\partial^{2} V}{\partial X^{2}}+\frac{\partial^{2} V}{\partial Y^{2}}+\frac{\partial^{2} V}{\partial Z^{2}}\right]-\rho_{f} \cdot g\left[\beta_{T} \cdot \Delta T \cdot \theta-\beta_{C} \cdot \Delta C . C\right]
\end{aligned}
$$

multiply both sides by $\frac{L^{2}}{\mu \cdot u_{0}}$ and then get :

$$
\begin{aligned}
\operatorname{Re}\left[\frac{\partial V}{\partial t}+U \frac{\partial V}{\partial X}\right. & \left.+V \frac{\partial V}{\partial Y}+W \frac{\partial V}{\partial Z}\right] \\
& =-\frac{\partial P}{\partial Y}+\left[\frac{\partial^{2} V}{\partial X^{2}}+\frac{\partial^{2} V}{\partial Y^{2}}+\frac{\partial^{2} V}{\partial Z^{2}}\right]-\frac{\rho \cdot g \cdot L^{2}}{\mu \cdot u_{0}}\left[\beta_{T} \cdot \Delta T \theta-\beta_{C} \cdot \Delta C \cdot C\right]
\end{aligned}
$$

And

$$
\begin{gathered}
\frac{\rho^{2} \cdot g \cdot L^{3} \cdot \beta_{T} \cdot \Delta T \cdot \theta}{\mu \cdot u_{0} \cdot \rho \cdot L}-\frac{\rho^{2} \cdot g \cdot L^{3} \cdot \beta_{T} \cdot \Delta C \cdot C}{u_{0} \cdot \rho \cdot L}=\frac{G r_{T}}{R e} \theta-\frac{G r_{C}}{R e} C \\
N=\frac{G r_{C}}{G r_{T}}
\end{gathered}
$$

and then :

$$
R e\left[\frac{\partial V}{\partial t}+U \frac{\partial V}{\partial X}+V \frac{\partial V}{\partial Y}+W \frac{\partial V}{\partial Z}\right]=-\frac{\partial P}{\partial Y}+\left[\frac{\partial^{2} V}{\partial X^{2}}+\frac{\partial^{2} V}{\partial Y^{2}}+\frac{\partial^{2} V}{\partial Z^{2}}\right]+\frac{G r_{T}}{R e}[\theta-N C]
$$

Lastly in the $\mathrm{Z}$ direction:

$\rho\left[\frac{\partial v}{\partial t}+u \frac{\partial w}{\partial x}+v \frac{\partial w}{\partial y}+w \frac{\partial w}{\partial z}\right]=-\frac{\partial p}{\partial x}+\mu\left[\frac{\partial^{2} w}{\partial x^{2}}+\frac{\partial^{2} w}{\partial y^{2}}+\frac{\partial^{2} w}{\partial z^{2}}\right]$ 
The thermal energy conservation equation for the fluid layer becomes as follows:

$$
(\rho C p)_{f}[\frac{\partial T}{\partial t}+u \underbrace{\left.\frac{\partial T}{\partial x}+v \frac{\partial T}{\partial y}+w \frac{\partial T}{\partial z}\right]}_{A}=k_{f}\left[\frac{\partial^{2} T}{\partial x^{2}}+\frac{\partial^{2} T}{\partial y^{2}}+\frac{\partial^{2} T}{\partial y^{2}}\right]
$$

Term $(A)$ is the rate of change of the temperature within the medium due to the convection of fluid into it. Once multiplied by $\left(\rho c_{p}\right)_{f}$, it becomes the rate of change of thermal energy per unit volume of fluid, due to convection.

Non-dimension form of energy equation:

$$
\begin{gathered}
(\rho . C p)_{f}\left[\frac{\partial(\Delta T . \theta)}{\partial \frac{t . L}{u_{0}}}+\left(U . u_{0}\right) \frac{\partial(\Delta T . \theta)}{\partial(X . L)}+\left(V \cdot u_{0}\right) \frac{\partial(\Delta T . \theta}{\partial(Y . L)}+\left(W . u_{0}\right) \frac{\partial(\Delta T . \theta)}{\partial(Z . L)}\right] \\
=K_{f}\left[\frac{\partial^{2}(\Delta T . \theta)}{\partial(X . L)^{2}}+\frac{\partial^{2}(\Delta T . \theta)}{\partial(Y . L)^{2}}\right] \\
(\rho . C p)_{f}\left(\frac{\Delta T . u_{0}}{L}\right)\left[\frac{\partial \theta}{\partial t}+U \frac{\partial \theta}{\partial X}+V \frac{\partial \theta}{\partial Y}+W \frac{\partial \theta}{\partial Z}\right]=\frac{K_{f} \cdot \Delta T}{L^{2}}\left[\frac{\partial^{2} \theta}{\partial X^{2}}+\frac{\partial^{2} \theta}{\partial Y^{2}}+\frac{\partial^{2} \theta}{\partial Z^{2}}\right]
\end{gathered}
$$

multiply both sides by $\frac{L^{2}}{K_{f} \cdot \Delta T}$ and then get :

$$
\begin{aligned}
& \frac{\rho_{f} \cdot L \cdot u_{0} \cdot C p_{f} \cdot \mu}{\mu \cdot K_{f}}\left[\frac{\partial \theta}{\partial t}+U \frac{\partial \theta}{\partial X}+V \frac{\partial \theta}{\partial Y}+W \frac{\partial \theta}{\partial Z}\right]=\left[\frac{\partial^{2} \theta}{\partial X^{2}}+\frac{\partial^{2} \theta}{\partial Y^{2}}\right] \\
& \operatorname{Re} \cdot \operatorname{Pr}\left[\frac{\partial \theta}{\partial t}+U \frac{\partial \theta}{\partial X}+V \frac{\partial \theta}{\partial Y}+W \frac{\partial \theta}{\partial Z}\right]=\left[\frac{\partial^{2} \theta}{\partial X^{2}}+\frac{\partial^{2} \theta}{\partial Y^{2}}+\frac{\partial^{2} \theta}{\partial Z^{2}}\right]
\end{aligned}
$$


The mass (concentration) balance equation in fluid layer can expressed as follows:

Mass Equation:

$$
\rho_{f}\left[\frac{\partial C}{\partial t}+u \frac{\partial C}{\partial x}+v \frac{\partial C}{\partial y}+w \frac{\partial C}{\partial z}\right]=\rho_{f} \cdot D_{m}\left[\frac{\partial^{2} C}{\partial x^{2}}+\frac{\partial^{2} C}{\partial y^{2}}+\frac{\partial^{2} C}{\partial z^{2}}\right]-\rho_{f} \cdot D_{T}\left[\frac{\partial^{2} T}{\partial x^{2}}+\frac{\partial^{2} T}{\partial y^{2}}+\frac{\partial^{2} T}{\partial z^{2}}\right]
$$

Substitute the dimensional variables and then get:

$$
\begin{gathered}
\rho_{f}\left[\frac{\Delta C \cdot u_{0}}{\partial \frac{t . L}{u_{0}}}+\left(U \cdot u_{0}\right) \frac{\Delta C \cdot \partial C}{\partial(X . L)}+\left(V \cdot u_{0}\right) \frac{\Delta C \cdot \partial C}{\partial(Y . L)}+\left(W \cdot u_{0}\right) \frac{\Delta C \cdot \partial C}{\partial(Z . L)}\right] \\
=\rho \cdot D_{m}\left[\frac{\partial^{2}(\Delta C \cdot C)}{\partial(X . L)^{2}}+\frac{\partial^{2}(\Delta C \cdot C)}{\partial(Y . L)^{2}}+\frac{\partial^{2}(\Delta C . C)}{\partial(Z . L)^{2}}\right]-\rho D_{T}\left[\frac{\partial^{2}(\Delta T \cdot \theta)}{\partial(X . L)^{2}}+\frac{\partial^{2}(\Delta T \cdot \theta)}{\partial(Y \cdot L)^{2}}+\frac{\partial^{2}(\Delta T \cdot \theta)}{\partial(Z . L)^{2}}\right]
\end{gathered}
$$

multiply both sides by $\left(\frac{L}{\rho_{f} . \Delta C . u_{0}}\right)$ and then get :

$$
\begin{aligned}
& \frac{\partial C}{\partial t}+U \frac{\partial C}{\partial X}+V \frac{\partial C}{\partial Y}+W \frac{\partial C}{\partial Z}=\frac{D_{m}}{u_{0} \cdot L}\left[\frac{\partial^{2} C}{\partial X^{2}}+\frac{\partial^{2} C}{\partial Y^{2}}+\frac{\partial^{2} C}{\partial Z^{2}}\right]-\frac{D_{T} \cdot \Delta T}{L \cdot u_{0} \cdot \Delta C}\left[\frac{\partial^{2} \theta}{\partial X^{2}}+\frac{\partial^{2} \theta}{\partial Y^{2}}+\frac{\partial^{2} \theta}{\partial Z^{2}}\right] \\
& A=\frac{D_{m} \cdot v}{u_{0} \cdot L \cdot v}\left[\frac{\partial^{2} C}{\partial X^{2}}+\frac{\partial^{2} C}{\partial Y^{2}}+\frac{\partial^{2} C}{\partial Z^{2}}\right]=\frac{1}{S c \cdot R e}\left[\frac{\partial^{2} C}{\partial X^{2}}+\frac{\partial^{2} C}{\partial Y^{2}}+\frac{\partial^{2} C}{\partial Z^{2}}\right]
\end{aligned}
$$




$$
\begin{gathered}
B=\frac{D_{T} \cdot \Delta T}{L \cdot u_{0} \cdot \Delta C}\left[\frac{\partial^{2} \theta}{\partial X^{2}}+\frac{\partial^{2} \theta}{\partial Y^{2}}+\frac{\partial^{2} \theta}{\partial Z^{2}}\right]=\frac{D_{m} \cdot S_{T} \cdot \Delta T}{L \cdot u_{0} \cdot \Delta C}\left[\frac{\partial^{2} \theta}{\partial X^{2}}+\frac{\partial^{2} \theta}{\partial Y^{2}}+\frac{\partial^{2} \theta}{\partial Z^{2}}\right]=\frac{\alpha}{S c \cdot R e}\left[\frac{\partial^{2} \theta}{\partial X^{2}}+\right. \\
\left.\frac{\partial^{2} \theta}{\partial Y^{2}}+\frac{\partial^{2} \theta}{\partial Z^{2}}\right]
\end{gathered}
$$

$$
\frac{\partial C}{\partial t}+U \frac{\partial C}{\partial X}+V \frac{\partial C}{\partial Y}+W \frac{\partial C}{\partial Z}=\frac{1}{S c \cdot R e}\left[\frac{\partial^{2} C}{\partial X^{2}}+\frac{\partial^{2} C}{\partial Y^{2}}+\frac{\partial^{2} C}{\partial Z^{2}}\right]-\frac{\alpha}{S c \cdot R e}\left[\frac{\partial^{2} \theta}{\partial X^{2}}+\frac{\partial^{2} \theta}{\partial Y^{2}}+\frac{\partial^{2} \theta}{\partial Z^{2}}\right]
$$




\section{APPENDIX B}

\section{Heaviside Function}

\section{B.1 Introduction}

As described in chapter 5, COMSOL Metaphysics uses sets of partial differential equations to solve a wide range of engineering problems. COMSOL employs a set of built-in modes to perform numerical analysis in several areas of physics, including fluid mechanics, acoustics, diffusion, structural mechanics, electromagnetics, and heat transfer. Multiple physics modes can be joint with each other or with user created differential equations to consider complex problem that experience changes in multiple ways.

\section{B.2 Heaviside Function}

There are multiple techniques of representing a delta throughout a finite domain. Two techniques were discovered: the linear interpolation technique and the smooth Dirac delta function technique. The linear interpolation technique demonstrates the heat capacity of the material over phase change as a linear increase to a maximum followed by a linear decrease at an identical but negative slope back to the original value. Indicating the maximum heat capacity needs first verifying the temperature span of the phase transition. When the range is fixed, matching the integral of heat capacity through the final melting temperature span to the given latent heat value define the maximum heat capacity value. This technique has already been used in COMSOL to

pure materials with point melting temperature adjusted from study of the heat capacities of 
biological materials. The Heaviside step function is a mathematical function signified $H(x)$, or sometimes $\theta(x)$ or $u(x)$ [188], and also accepted as the "unit step function". The term "Heaviside step function” and its symbol can denote either a piecewise constant function or a generalized function.
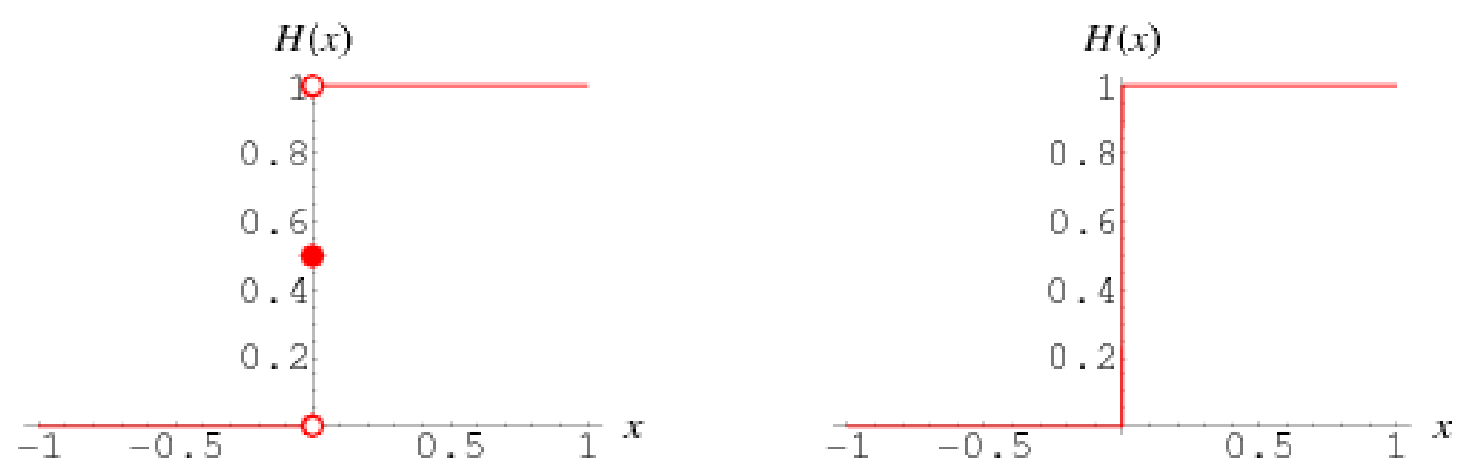

When explained as a piecewise constant function, the Heaviside step function is known by

$$
H(x)=\left[\begin{array}{ll}
0 & x<0 \\
\frac{1}{2} & x=0 \\
1 & x>0
\end{array}\right]
$$

[189]. The plot above depicts this function (left figure), and how it would develop if showed on an oscilloscope (right figure).

When expressed as a generalized function, it can be stated as a function $\theta(x)$ such that $\int \theta(x) \emptyset^{\prime}(x) d x=-\emptyset(0)$

For $\emptyset^{\prime}(x)$ the derivative of a adequately smooth function $\emptyset(x)$ that declines sufficiently rapidly [190]. Heaviside generalized function might be presents as HeavisideTheta, when using UnitStep 
to state the piecewise function Piecewise $[\{\{1, x \geq 0\}\}]$ (which, it should be noticed, accepts the convention $H(0)=1$ rather than the conventional definition $H(0)=\frac{1}{2}$ ).

The shorthand note $H_{c}(x) \equiv H(x-c)$ is sometimes also applied.

The Heaviside step function is correlated to the boxcar function by $\Pi(x)=H\left(x+\frac{1}{2}\right)-H(x-$ $\left.\frac{1}{2}\right)$

and can be expressed in terms of the sign function by $H(x)=\frac{1}{2}[1+\operatorname{sgn}(x)]$

The derivative of the step function is shown by $\frac{d}{d x} H(x)=\delta(x)$,

Where $\delta(x)$ is the delta function [191].

The Heaviside step function is associated to the ramp function $R(x)$ by $R(x)=x H(x)$, and to the derivative of $R(x)$ by $\frac{d}{d x} R(x)=H(x)$. The two are also linked through $R(x)=$ $H(x) * H(x)$ where * indicates convolution.

Bracewell [192] gives several identities, some of which include the following. Letting * indicates the convolution, $H(x) * f(x)=\int_{-\infty}^{x} f\left(x^{\prime}\right) d x^{\prime}$

$H(t) * H(t)=\int_{-\infty}^{\infty} H(u) H(t-u) d u=H(0) \int_{0}^{\infty} H(t-u) d u=H(0) H(t) \int_{0}^{1} d u=t H(t)$

in addition $H(a x+b)=H\left(x+\frac{b}{a}\right) H(a)+H\left(-x-\frac{b}{a}\right) H(-a)=\left\{\begin{array}{cc}H\left(x+\frac{b}{a}\right) & a>0 \\ H\left(-x-\frac{b}{a}\right) & a<0\end{array}\right.$ 


$$
\begin{aligned}
& H(x)=\lim _{t \rightarrow 0}\left[\frac{1}{2}+\frac{1}{\pi} \tan ^{-1}\left(\frac{x}{t}\right)\right] \\
& =\frac{1}{\sqrt{\pi}} \lim _{t \rightarrow 0} \int_{-x}^{\infty} t^{-1} e^{-u^{2} / t^{2}} d u=\frac{1}{2} \lim _{t \rightarrow 0} \operatorname{erfc}\left(-\frac{x}{t}\right)=\frac{1}{\pi} \lim _{t \rightarrow 0} \int_{-\infty}^{x} t^{-1} \operatorname{sinc}\left(\frac{u}{t}\right) d u \\
& =\frac{1}{\pi} \lim _{t \rightarrow 0} \int_{-\infty}^{x} \frac{1}{u} \sin \left(\frac{u}{t}\right) d u=\frac{1}{2}+\frac{1}{\pi} \lim _{t \rightarrow 0} \operatorname{si}\left(\frac{\pi x}{t}\right)=\lim _{t \rightarrow 0}\left\{\begin{array}{cl}
\frac{1}{2} e^{x / t} & \text { for } x \leq 0 \\
1-\frac{1}{2} e^{-x / t} & \text { for } x \geq 0
\end{array}\right. \\
& =\lim _{t \rightarrow 0} \frac{1}{1+e^{-x / t}}=\lim _{t \rightarrow 0} e^{-e^{\frac{-x}{t}}} \\
& =\frac{1}{2} \lim _{t \rightarrow 0}\left[1+\tanh \left(\frac{x}{t}\right)=\lim _{t \rightarrow 0} \int_{-\infty}^{x} t^{-1} \Lambda\left(\frac{x-\frac{1}{2} t}{t}\right) d x\right]
\end{aligned}
$$

Where $\operatorname{erfc}(x)$ is the erfc function, $\operatorname{si}(x)$ is the sine integral, $\operatorname{sinc}(x)$ is the Sinc function, and $\Lambda(x)$ is the one-argument triangle function. The first four of these are explained above for $t=0.2,0.1$, and 0.01 .

Obviously, any monotonic function with continuous unequal horizontal asymptotes is a Heaviside step function under suitable scaling and possible indication. The Fourier transform of the Heaviside step function is defined by:

$$
F[H(x)]=\int_{-\infty}^{\infty} e^{-2 \pi i k x} H(x) d(x)=\frac{1}{2}\left[\delta(x)-\frac{i}{\pi k}\right]
$$




\section{REFERENCES}

[1] W. E. Langlois, “Buoyancy-Driven Flows in Crystal-Growth Melts” Ann. Rev. Fluid Mech., Vol. 17, pp.191-215, 1985.

[2] J. Hamacher, “Analysis of Microgravity Measurements Performed during DI”, European Space Agency, (Special Publication) ESA SP, pp. 413-420, 1987.

[3] R. Kita, S. Wiegand, and J. Luettmer-Strathmann, "Sign Change of the Soret Coefficient of Poly (Ethlene Oxide) in Water/Ethanol Mixtures Obsvered by Thermal Diffusion Forced Rayleigh Scattering”J. Chem. Phys., Vol.121, No. 8, pp. 3874-3886, 2004.

[4] A. Abbasi, Z. Saghir and M. Kawaji, "Evaluation of the ratio of the evaporation energy to the activation energy for estimating the thermodiffusion coefficient”, J. Non-Equilib. Thermodyn., Vol. 35, No. 1, pp. 1-14, 2010.

[5] M. E. Schimpf. "Studies in thermodiffusion by thermal field-flow fractionation”, Proceedings of the Third International Symposium on Thermodiffusion, pp. 58-63, 1998.

[6] S. R. De Groot, “Non-Equilibrium Thermodynamics”, Dover Publication Inc., New York, 1984.

[7] P. Georis, F. Ontel, S. Van Vaerenbergh, Y. Decroly and J. C. Legros, "Measurement of the Soret coefficient in crude oil”, In Proceedings of the European Petroleum Conference, pp. 150-161, 1998.

[8] B. N. Bhat and R. A. Swalin, "Modelling of Precipitation Hardening in Casting Aluminium Alloys”, Scripta Metallurgica, Vol. 6, pp. 523-528, 1972.

[9] S. Van Vaerenbergh, J. C. Legros, J. L. Daridon, T. Karapantsios, M. Kostoglou, and Z.Saghir, "Multicomponent transport studies of crude oils and asphaltenes in DSC 
Program”, Microgravity Science and Technology, Vol.18, No. 3-4, pp.150-154, 2006.

[10] G. Galliéro, J. Colombani, P. A. Bopp, B. Duguay, J. P. Caltagirone and F. Montel, "Thermal diffusion in micropores by molecular dynamics computer simulations”, Physica A: Statistical Mechanics and its Applications, Vol. 361, No. 2, pp. 494-510, 2006.

[11] Z. Saghir, C.G. Jiang, M. Chacha, Y. Yan, M. Khawaja and S. Pan, "Thermodiffuison in porous media. In Transport Phenomena in Porous Media III, Elsevier Publication Inc., Oxford, pp. 227-260, 2005.

[12] A. Ott, "Isotope Seperation by Thermal Diffusion in Liquid Metal”, Science, Vol. 164, No. 3877, pp. 297, 1969.

[13] M. Sugisaki, K. Idemitsu, S. Mukai and H. Furuya, "Thermal diffusion of Tritium in NB Metal”, J. Nucl. Mater., Vol. 104, pp. 1493-1497, 1981.

[14] E. Jafar-Salehi, M. Eslamian and Z. Saghir, "Gravity Effects in Mass and Solute Transport in a Binary Metallic System in the Presence of Thermodiffusion”, 64 ${ }^{\text {th }}$ International Astronautical Congress, Beijing, China, 2013.

[15] D. Reith and F. Mueller-Plathe, “On the nature of thermal diffusion in binary lennardjones liquids”, J. Chem. Phys., Vol.112, pp. 2436-2443, 2000.

[16] A. Bejan, “Convection Heat Transfer”, 1st ed., Wiley Publisher Inc., New York, 1984.

[17] M. Eslamian, F. Sabzi and Z. Saghir, "Modelling of Thermodiffusion in Liquid Metal alloys”, Phys. Chem. Chem. Phys., Vol. 12, pp. 13835- 13848, 2010.

[18] S. Srinivasan and Z. Saghir, "Experimental Approaches to Study Thermodifusion-A Review”, Int. J. Therm. Sci., Vol. 50, No. 7, pp. 1125-1137, 2011

[19] W. Enge, W Köhler, “Thermal Diffusion in a Critical Polymer Blend”, Phys. Chem. Chem. Phys., Vol. 6, pp. 2373-2378, 2004. 
[20] R. Piazza, S Iacopini and B. Triulzi, "Thermophoresis as a Probe of Case of Protein Solutions”, Phys. Chem. Chem. Phys., Vol. 6, pp. 1616-1622, 2004.

[21] J. Colombani, G. Gallie'ro, B. Duguay, J-P.Caltagirone, F. Montel and P. A. Bopp, “Thermal Diffusion in Micropores by Molecular Dynamics Computer Simulations”, Phys. Chem. Chem. Phys., Vol. 4, pp. 313-321, 2004.

[22] C. Balaji and S. P. Venkateshan, "Effect of Sinusoidal Heating on Natural Convection Coupled to Thermal Radiation in a Square Cavity Subjected to Cross Temperature Gradients”, Int. J. Heat Fluid Flow, Vol. 14, pp. 260-267, 1995.

[23] E. L. Dougherty and H. G. Drickamer, "Thermal Diffusion and Molecular Motion in Liquid”, J. Phys. Chem., Vol. 59, No.5, pp. 443, 1955.

[24] S. Kjelstrup and D. Bedeaux, "Non-Equilibrium Thermodynamics of Heterogeneous Systems”, World Scientific Publishing, 2008.

[25] E. Jafar-Salehi, M. Eslamian and Z. Saghir, "Estimation of Molecular and Thermodiffusion Coefficients for Non-Ideal Molten Metal Alloys and its Implication in Solidification Process”, CJCE, Vol. 92, No. 7, pp. 1163-1333, 2013.

[26] R. B. Bird, W. E. Stewart and E. N. Lightfoot, “Transport Phenomena” 2nd Ed, John Wiley and Sons Publisher Inc., pp. 765, 2002.

[27] R. Haase, “Thermodynamics of Irreversible Processes”, Addison-Wesley Publisher Inc., 1969.

[28] F. R. Winter and H. G. Drickamer, “Thermal Diffusion in Liquid Metal”, J. Phys. Chem., Vol. 59, No. 12, pp. 1229-1230, 1955.

[29] M. Eslamian and Z. Saghir, "Microscopic Study and Modeling Thermodiffusion in Associating Mixtures”, Physical Review E, Vol. 80, pp. 11201, 2009. 
[30] M. Eslamian and Z. Saghir, “A Critical Review of Thermodiffusion Models: Role and Significance of the Heat of Transport and Activation Energy of Viscouse Flow”, J. NonEquilib. Thermodyn., Vol. 34, No.2, pp. 97, 2009.

[31] D. Jaffe and P. G. Shewmon, "Thermal Diffusion of Substitutional Impurities in Copper, Gold and Silver”, Acta Metallurgica, Vol. 12, pp. 515-527, 1964.

[32] A. Lodding, “Thermotransport in Monatomic and Ionic Liquids, in Isotope Effects in Chemical Processes”, Advances in Chemistry, Vol. 89, Chapter 13, pp.264-274, 1969.

[33] V. Galina and M. Omini, "Vacancy as a Phonon Field Perturbation. I. Lattice Distorsion around a Vacancy”, IL Nuovo Cimento B, Vol. 8, No. 1, pp. 65-89, 1972.

[34] B. N. Bhat and R. A. Swalin, “Thermotransport of Silver in Liquid Gold”, Acta metallurgica, Vol. 20, pp. 1387-1396, 1972.

[35] P. Thernquist, “Force Transport of Impurities in Lithium. II. Thermotransport of the Noble Metals, Zinc, and Cadmium”, Phys. Status Solidi (a), Vol. 18, No. 1, pp. 255-264, 1973.

[36] H. J. V. Tyrrell, "Some Problems Associated with the Definition of the Heat of Transfer for Binary Liquid Systems”, Transaction of Faraday Society, Vol. 56, pp. 770-775, 1960.

[37] J. P. Praizey, "Benefits of Microgravity for Measuring Thermotransport Coefficient in Liquid Metallic Alloys”, Int. J. Heat Mass Transfer, Vol. 32, No.12, pp. 2385- 2401, 1989.

[38] Y. Malmejac and J. P. Praizey, “Thermomigration of Cobalt in liquid tin- Experiment 1 ES 320, Proceeding of Third European Symposium on Material Sciences in Space, Grenoble France, ESA SP-191, pp. 127-132, 1984.

[39] A. D. Payton and J. C. R. Turner, "Soret Coefficients and Heats of Transport of Some Salts of Alkaline Earth Metals in Water at $25^{\circ} \mathrm{C}^{\prime}$, Trans. Faraday Soc.,Vol. 58, pp. 55-59, 1962. 
[40] M. Gerl, "Contribution to the Calculation of Forces Acting on An Impurity Metal Subjected to a Temperature Gradient”, J. Phys. Chem. Solids, Vol. 28, No. 5, pp.725-736, 1967.

[41] R. C. Jones, "On the Theory of the Thermal Diffusion Coefficient for Isotopes”, Phys. Rev. A,Vol. 58, pp. 111-122, 1940.

[42] S. Chapman and T. G. Cowling, "The Mathematical Theory of Non-uniform Gases", Cambridge University Press, 1952.

[43] M. Shimoji, "Liquid Metals: An Introduction to the Physics and Chemistry of Metals in the Liquid State”, Academic Press, London, 1977.

[44] V. K. Ratti and E. Evans, "The Resistivity and Thermoelectric Power of the Liquid Alkaline Earth Metals”, J. Phys. F, Vol. 3, pp. L238-L243, 1973.

[45] S. Van Vaerenbergh, J. P. Garandet, J. P. Praizey and J. C. Legros, "Experimental Microgravity Soret Coefficients in Multicomponent System: towards mixing rules?”, Phys. Rev. E, Vol. 58, No. 2, pp. 1866-1873, 1998.

[46] C. Balaji and S. P. Venkateshan, "Interaction of Surface Radiation with Free Convection in a Square Cavity”, Int. J. Heat Fluid Flow, Vol.14, pp.260-267, 1993.

[47] C. Balaji and S. P. Venkateshan, "Correlations for Free Convection and Surface Radiation in a Square Cavity”, Int. J. Heat Fluid Flow, Vol.15, pp. 249-251, 1994.

[48] M. Akiyama and Q.P. Chong, "Numerical Analysis of Natural Convection with Surface Radiation in a Square Cavity”, Num. Heat Transfer, Vol. 31, pp. 419-433, 1997.

[49] N. Ramesh and S. P. Venkateshan, "Effect of Surface Radiation on Natural Convection in a Square Enclosure”, J. Thermophys. Heat Transfer, Vol. 13, pp. 299-301, 1999.

[50] E. H. Ridouane, M. Hasnaoui, A. Amahmid and A. Raji, "Effect of Surface Radiation on 
Natural Convection in a Square”, Num. Heat Transfer Fluid Flow, Vol. 45, pp. 289-311, 2004.

[51] C. Gururaja Rao, A. Venkata Krishna and P. Naga Srinivas, "Simulation Studies on Multimode Heat Transfer from a Square-Shaped Electronic Device with Multiple Discrete Heat Sources”, Num. Heat Transfer Fluid Flow, Vol. 48, pp. 427-446, 2005.

[52] A. Bahlaoui, A. Raji and M. Hasnaoui, "Combined Effect of Radiation and Natural Convection in a Rectangular Enclosure Discreetly Heated from One Side”, Int. J. Num. Meth. Heat Fluid Flow, Vol. 16, pp. 431-450, 2006.

[53] C. Y. Han and S. W. Baek, "The Effects of Radiation on Natural Convection in a Rectangular Enclosure Divided by Two Partitions”, Num. Heat Transfer Fluid Flow, Vol. 37, pp. 249-270, 2000.

[54] J. F. Hinojosa, C. A. Estrada, R. E. Cabanillas and G. Alvarez, "Numerical Study of Transient and Steady-State Natural Convection and Surface Thermal Radiation in a Horizontal Square Open Cavity”, Num. Heat Transfer Fluid Flow, Vol. 48, pp. 179-196, 2005.

[55] A. A. Dehghan and M. Behnia, "Combined Natural Convection-Conduction and Radiation Heat Transfer in a Discretely Heated Open Cavity”, J. Heat Transfer, Vol. 118, pp. 56-64, 1996.

[56] J. L. Lage and A. Bejan, "The Resonance of Natural Convection in an Enclosure Heated Periodically from the Side”, Int. J. Heat Mass Transfer, Vol. 36, pp. 2027-2038, 1993. 
[57] E. K. Lakhal, M. Hasnaoui, P. Vasseur and E. Bilgen, "Natural Convection in a Square Enclosure Heated Periodically from Part of the Bottom Wall”, Num. Heat Transfer. A, Vol. 27, pp. 319-333, 1995.

[58] E. K. Lakhal, M. Hasnaoui, and P. Vasseur, "Natural Convection Heat Transfer in Rectangular Cavities Partially Heated from Below”, Int. J. Heat Mass Transfer, Vol. 42, pp. 3927-3941, 1999.

[59] B. V. Antohe and J. L. Lage, "Amplitude Effect on Convection Induced by Time-Periodic Horizontal Heating”, Intl. J. Heat Mass Transfer, Vol. 39, pp. 1121-1133, 1996.

[60] B. V. Antohe and J. L. Lage, "The Prandtl Number Effect on the Optimum Heating Frequency of an Enclosure Filled with Fluid or with a Saturated Porous Medium”, Intl. J. Heat Mass Transfer, Vol. 40, pp. 1313-1323, 1997.

[61] H. S. Kwak and J. M. Hyun, "Numerical Simulation of Time-Dependent Buoyancy-Driven Convection in an Enclosure”, J. Fluid Mechanics, Vol. 329, pp. 65-88, 1996.

[62] H. S. Kwak, K. Kuwahara and J. M. Hyun, "Resonant Enhancement of Natural Convection Heat Transfer in a Square Enclosure”, Int. J. Heat Mass Transfer, Vol. 41, pp. 2837-2846, 1998.

[63] B. Abourida, M. Hasnaoui and S. Douamna, "Transient Natural Convection in a Square Enclosure with Horizontal Walls Submitted to Periodic Temperatures”, Num. Heat Transfer A, Vol. 36, No.7, pp. 737-750, 1999.

[64] C. Zhao and S. Valliappan, "Transient Infinite Elements for Contaminant Transport 
Problems”, Int. J. Num. Meth. Eng., Vol. 37, Issue 7, pp. 1143-1158, 1994.

[65] J. C. Petterson and J. Imberger, "Unsteady natural convection in a rectangular cavity", J. Fluid Mechanics, Vol. 100, No.1, pp. 65-86, 1980.

[66] K. Kublbeck, G. P. Merker and J. Straub, "Advanced Numerical Computation of Twodimensional Time-dependent Free Convection in Cavities”, Int. J. Heat Mass Transfer, Vol. 23, pp. 203-217, 1980.

[67] G. N. Ivey, "Experiments on Transient Natural Convection in a Cavity”, J. Fluid Mech., Vol. 144, pp. 389-401, 1984.

[68] S. G. Schladow, “Oscillatory Motion in a Side-heated Cavity”, J. Fluid Mech., Vol. 213, pp. 589-610, 1990.

[69] J. C. Petterson and S. W. Armfield, "Transient Features of Natural Convection in a Cavity”, J. Fluid Mech., Vol. 219, pp. 469-497, 1990.

[70] C. G. Jeevaraj and J. C. Patterson, "Experimental Study of Transient Natural Convection of Glycerol-Water Mixtures in a Side Heated Cavity”, Int. J. Heat Mass Transfer, Vol. 35, No. 6, pp. 1573-1587, 1992.

[71] J. N. Koster, “Time Dependent Heat Transfer in Hele-Shaw Slots”, Intl. Comm. Heat and Mass Transfer, Vol. 12, No. 2, pp. 159-167, 1985.

[72] J. N. Koster and U. Muller, “Oscillatory Convection in Vertical Slots”, J. Fluid Mech., Vol. 39, pp. 363-390, 1984.

[73] M. Kazmierczak and Z. Chinoda, "Buoyancy-Driven Flow in an Enclosure with Time Periodic Boundary Conditions”, Intl. J. Heat Mass Transfer, Vol. 35, No. 6, pp. 1507-1518, 1992.

[74] F. T. Poujol, J. Rojas and E. Ramos, “Natural Convection of a High Prandtl Number Fluid 
in a Cavity”, Intl. Comm. Heat Mass Transfer, Vol. 27, No. 1, pp.109-118, 2000.

[75] M. K. Higgins, PIV Study o f Oscillating Natural Convection Flow within a Horizontal Rectangular Enclosure, M.S. thesis, Washington State University, 2000.

[76] A. djiba, M. Mamou and P. Vasseur, "Onset of Double-Diffusive Convection in a Rectangular Porous Cavity Subject to Mixed Boundary Conditions”, Intl. J. Heat Mass Transfer, Vol. 43, No. 9, pp. 1505-1522, 2000.

[77] K. Sundaravadivelu and P. Kandaswamy, "Double Diffusive Nonlinear Convection in a Square Cavity”, Fluid Dynamics Research, Vol. 27, No. 5, pp. 291-303, 2000.

[78] T. C. Jue and B. Ramaswamy, "Numerical Analysis of Thermosolutal Flows in a Cavity with Gravity Modulation effects”, Heat and Mass Transfer/Waerme- und Stoffuebertragung, Vol. 38, No. 7-8, pp. 665-672, 2002.

[79] M. Rappaz, "Modeling of Microstructure Formation in Solidification Processes”, Intl. Mater. Rev., Vol. 34, No. 3, pp. 93-123, 1989.

[80] C. Beckermann and R. Viskanta, "Mathematical Modeling of Transport Phenomena During Alloy Solidification”, Appl. Mech. Rev., Vol. 46, pp. 1-27, 1993.

[81] W.W. Mullins and R.F. Sekerka, "Stability of a Planar Interface During Solidification of a Dilute Binary Alloy”, J. Appl. Phys., Vol. 35, pp. 444, 1964.

[82] J. R. sazarin and A. Hellawell, "Minimizing segregation during the controlled directional solidification”, Metall. Trans. A, Vol. 19A, pp. 1861, 1998.

[83] S. N. Tewari and R. Shah, "Macrosegregation During Dendritic Arrayed Growth of Hypoeutectic Pb-Sn Alloys: Influence of Primary Arm Spacing and Mushy Zone Length” Metall. Trans. A., Vol. 23A, pp. 3382, 1992. 
[84] S. D. Felicelli, J. C. Heinrich and D. R. Poirier, "Simulation of freckles during vertical solidification of binary alloys”, Metall. Trans. B., Vol. 22B, pp. 847, 1991.

[85] S. D. Felicelli, J. C. Heinrich and D. R. Poirier, "Three-dimensional simulations of freckles in binary alloys”, J. Cryst. Growth., Vol. 191, pp. 879, 1998.

[86] A. K. Singh, Influence of Thermosolutal Convection on Macrosegregation During Solidification of Binary Alloys, PhD Thesis, IIT Kanpur, Kanpur, 2003.

[87] A. K. Singh, B. Basu and A. Ghosh, "Mathematical Modelling of Convection and Macrosegration During Casting of Metallic Alloys”, International Journal of Engineering Systems Modelling and Simulation, Vol. 2, pp. 226-233, 2010.

[88] Y. Kawaguchi, H.Yasuda, Y. Okano and S. Dost, “Numerical Simulation of Crystal Growth of CdZnTe by Vertical Gradient Freezing Method -Crucible Rotation Effect” Int. J. Transport Phenomena, Vol. 7, pp. 175-187, 2005.

[89] Y.Okano, H. Kondo and S. Dost, "Numerical study of interface shape control in the VGF growth of compound semiconductor crystal”, J. Cryst. Growth, Vol. 237, pp. 1769-1772, 2002.

[90] P. Barvinschi and F. Barvinschi, "Coupled Heat Transfer and Fluid Dynamics Modeling of InSb Solidification”, AIP Conference Proceeding, pp. 190-197, 2011.

[91] M. Shemirani, “A Numerical Modeling Study on the Effects of both Radial and Axial Temperature Gradient on a Large Diameter Si0.25Ge0.75 Alloy in a Bridgman Setting”, Ph.D Thesis, Ryerson University, Canada, 2012.

[92] M. Vynnycky and S. Kimura, "An Analytical and Numerical Study of Coupled Transient Natural Convection and Solidification in a Rectangular Enclosure”, Int. J. Heat Mass Transfer, Vol. 50, pp. 5204-5214, 2007. 
[93] F. Mechighel, S. Dost, B. Pateyron, M. El Ganaoui and M. Kadja, "Modeling of Silicon Transport into Germanium Using a Simplified Crystal Growth Technique”, COMSOL Proceeding Conference, pp. 69-76, Milan, 2009.

[94] D. Celentano, M. Cruchaga, N. Moraga and J.Fuentes, "Mathematical Modelling of Temperature Profile of Volcanic Soils Affected by an External Thermal Impact”, Numer. Heat Transfer, Part A, Vol. 39, pp. 631-654, 2001.

[95] M. A. Rady and A. K. Mohanty, "Natural Convection During Melting and Solidification of Pure Metals in a Cavity”, Num. Heat Transfer, Part A, Vol. 29, pp.49-63, 1996.

[96] V. R. Voller and C. Prakash, “A Fixed Grid Numerical Modeling Methodology for Convection-diffusion Mushy Region Phase-change Problems”, Intl. J. Heat Mass Transfer, Vol. 30, No.8, pp.1709-1719, 1987.

[97] V.R. Voller, N. C. Markatos and M. Cross, “Techniques for Accounting for the Moving Interface in Convection/Diffusion Phase Change”, In Numerical Methods in Thermal Problems, Pineridge press, Swansea, Vol. 4, pp. 595-609, 1985.

[98] V. R. Voller, N. C. Markatos and M. Cross, Solidifications of Fluid Flow and Heat/Mass Transfer Processes, Springer Publisher, Berlin, pp. 425-432, 1986.

[99] V. R. Voller, M. Cross and N. C. Markatos, “An Enthalpy Method for Convection/Diffusion Phase Changes”,Inlt. J. Numer. Meth. Eng., Vol. 24, pp. 271-284, 1987.

[100] D. K. Gartling, Finite Element Analysis of Convective Heat Transfer Problems with Change of Phase. In Computer Methods in Fluids, Pentech Publisher, London, pp. 257284, 1980. 
[101] S. B. Willers, P. A. Nikrityuk, K. Eckert, U. Michel and G. Zouhar, “Application of a Rotating Magnetic Field During Directional Solidification of Pb-Sn Alloys: Consequences on the CET”, Mater. Sci. Eng., A 413-414, pp. 211-216, 2005.

[102] G. S. Cole and G. F. Bolling, "Augmented Natural Convection and Equiaxed Grain Structure in Casting”, T. Metall. Soc., AIME 36, pp. 1366-1368, 1966.

[103] W. D. Griffiths and D. G. McCartney, "The Effect of Electromagnetic Stirring During Solidification on the Structure of Al-Si alloys”, Mater. Sci. Eng., A 216, pp. 47-60, 1996.

[104] J. K. Roplekar and J. A. Dantzig, “A Study of Solidification with a Rotating Magnetic Field”, Intl. J. Cast Metals Research, Vol. 14, pp. 79-98, 2001.

[105] B. Willers, S. Eckert, U. Michel, I. Haase and G. Zouhar, "The Columnar-to-Equiaxed Transition in Pb-Sn Alloys Affected by Electromagnetically Driven Convection”, Mater Sci. Eng. A, Vol. 402, pp. 55-65, 2005.

[106] M. Wu, A. Vakhrushev, G. Nummer, C. pfeiler, A. Kharicha and A. Ludwig, "Importance of Melt Flow in Solidifying Mushy Zone”, The open Transport Phenomena Journal, Vol. 2, pp. 16-23, 2010.

[107] V. R. Voller, A. D. Brent and C. Prakash, "The Modelling of Heat, Mass and Solute Transport in Solidification System”, Intl. J. Heat Mass Transfer, Vol.32, No. 9, pp. 1719, 1989.

[108] V. R. Voller, A. D. Brent and C. Prakash, "Modeling the Mushy Region in a Binary Alloy”, Appl. Math. Modeling, Vol. 14, pp. 320-326, 1990.

[109] V. R. Voller and C. R. Swaminathan, "General Source-Based Method for Solidification Phase Change”, Numer. Heat Transfer, Part B, Vol. 19, pp. 175-189, 1991. 
[110] P.J. Prescott and F. P. Incropera, "The Effect of Turbulence on Solidification of a Binary Metal Alloy with Electromagnetic Stirring”, In the Transport Phenomena in Materials Processing and Manufacturing, ASME HTD, Vol. 280, pp. 59-69, 1994.

[111] P. J. Prescott, and F. P. Incropera, "Convective Transport Phenomena and Macrosegregation During Solidification of a Binary Metal Alloy: I-Numerical Predictions”, J. Heat Transfer, Vol. 116, Issue. 3, pp. 735-741, 1994.

[112] A. L. Maples and D. R. Poirier, "Convection in the Two-Phase Zone of Solidifying Alloys”, Metall. Trans. B, Vol. 15B, pp. 163, 1984.

[113] P.A. Nikrityuk, K. Eckert, R. Grundmann, B. Willers and S. Eckert, "Modeling and Measurements of heat Transfer Phenomena in Two-Phase PbSn Alloy Solidification in an External Magnetic Field”, J. Therm. Sci., Vol. 12, No. 4, pp. 357-362, 2003.

[114] P. J. Presscot, “Convection Transport Phenomena During Solidification of Binary Metal Alloys and the Effects of Magnetic Fields”, Ph.D Thesis, Purdue University, West Lafayatte, IN, 1992.

[115] P. J. Presscott, F. P. Incropera and D. R. Gaskell, "Influence of Electromagnetic String on the Solidification of a Binary Metal Alloy”, Experimental Heat Transfer, Vol. 9, pp. 105131, 1996.

[116] P. J. Presscott and F. P. Incropera, "Magnetically Damped Convection During Solidification of a Binary Metal Alloy”, J. Heat Transfer, Vol. 115, pp. 302-310, 1993.

[117] P. J. Presscott and F. P. Incropera, "The effect of Turbulence on Solidification of a Binary Metal Alloy With Electromagnetic Stirring”, J. Heat Transfer, Vol. 117, pp. 716-724, 1995. 
[118] F. Mechighel, M. El Ganaoui, B.Pateyron, M.Kadja and S. Dost, "Finite Element Modeling of Silicon Transport into Germanium Using a Simplified Crystal Growth Technique”, Defect and Diffusion Forum, Vols. 312-315, pp. 240-247, 2011.

[119] T. L. Marin, "Solidification of a Liquid Metal Droplet Impinging on a Cold Surface", Proceedings of the COMSOL, Users Conference, pp. 246-253, Boston, 2006.

[120] M. Pasandideh-Fard, R. Bhola, S. Chandra and J. Mostaghimi, "Deposition of Tin Droplets on a Steel Plate: Simulation and Experiments”, Intl. J. Heat Mass Transfer, Vol.41, pp. 2929-2945, 1998.

[121] M. Pasandideh-Fard, S. D. Aziz, S. Chandra and J. Mostaghimi, "Cooling Effectiveness of a Water Drop Impinging on a Hot Surface”, Intl J. Heat Fluid Flow, Vol. 22, pp.201-210, 2001.

[122] J. C. Heinrich, S. Felicelli and D. R. Poirier, "Vertical Solidification of Dendritic Binary Alloys”, Computer Methods in Applied Mechanics and Engineering, Vol. 89, pp. 435-461, 1991.

[123] S. R. Coriell, M. R. Cordes, W. J. Boettinger and R. F. Sekerka, "Convective and Interfacial Instabilities During Unidirectional Solidification of a Binary Alloy”, J. Cryst. Growth, Vol. 49, pp. 13-28, 1980.

[124] G. B. McFadden, R. G. Rehm, S. R. Coriell, W. Chuck and K. A. Morrish, "Thermosolutal Convection During Directional Solidification”, Metallurgical Transaction A, Vol. 15A, pp. 2125-2137, 1984.

[125] P. Nandapurkar, D. R. Poirier, J. C. Heinrich and S. Felicelli, “Thermosolutal Convection During Dendritic Solidification of Alloys: Part I. Linear Stability Analysis”, Metall. Trans. B, Vol. 20B, pp.711-721, 1989. 
[126] I. L. Ferreira, V. R. Voller, B. Nestler and A. Garcia, “Two-Dimensional Numerical Model for the Analysis of Macrosegregation During Solidification”, Comp. Mater. Sci., Vol. 46, pp. 358-366, 2009.

[127] M. C. Schneider and C. Beckermann, "Formation of Macrosegregation by Multicomponent Thermosolutal Convective During the Solidification of Steel”, Metall. Mater. Trans. A, Vol. 26A, pp. 2373-2388, 1995.

[128] M. J. Krane, F. P. Incropera and D. R. Gaskell, “Solidification of ternary metal alloys. Part II: Predictions of convective phenomena and solidification behavior in Pb-Sb-Sn alloys”, Intl. J. Heat Mass Transfer, Vol. 40, pp. 3827-3835, 1997.

[129] A. P. Boeira, I. L. Ferreira and A. Garcia, "Influence of Solidification Rates on a Directional Solidification Process for the Production of Functionally Graded Materials”, Mater. Sci. Eng. A, Vol. 435, pp. 150-157, 2006.

[130] V. R. Voller, A. Mouchmov and M. Cross, "An Explicit Scheme for Coupling Temperature and Concentration Fields in Solidification Models”, Appl. Math. Modelling, Vol. 28, pp. 79-94, 2004.

[131] D. V. Alexandrov and D. L. Aseev, "One-dimensional Solidification of an Alloy with a Mushy Zone: Thermodiffusion and Temperature-Dependent Diffusivity”, J. Fluid Mechanics, Vol. 527, pp. 57-66, 2005.

[132] D. V. Alexandrov and D. L. Aseev, "Directional Solidification with a Two-Phase Zone: Thermodiffusion and Temperature-Dependent Diffusivity”, Comp. Mater. Sci.,Vol. 37, pp.1-6, 2006.

[133] D. V. Alexandrov, "Solidification with a Quasi Equilibrium Mushy Region: Analytical Solution of Nonlinear Model”, J. Cryst. Growth, Vol. 222, pp. 816-821, 2001. 
[134] P. A. Nikrityuk, K. Eckert and R. Grundmann, "A Numerical Study of Unidirectional Solidification of a Binary Metal Alloy under Influence of a Rotating Magnetic Field International”, J. Heat Mass Transfer, Vol. 49, pp. 1501-1515, 2006.

[135] S. Van Vaerenbergh, S. R. Coriell and G. B. McFadden, "Morphological Stability of a Binary Alloy: Thermodiffusion and Temperature- Dependent Diffusivity”, J. Cryst. Growth, Vol. 223, pp. 565-572, 2001.

[136] A. K. Singh, R. Pardeshi and B. Basu, "Modeling of Convection During Solidification of Metal and Alloys, Sadhana Journal, Vol. 26, Parts 1\&2, pp. 139-162, 2001.

[137] S. D. Ridder, S. Kou and R. Mehrabian, "Effect of Fluid Flow on Macrosegregation in Axi-Symmetric Ingots”, Metall. Trans. B, Vol.12B, pp. 435-447, 1981.

[138] D. Maier, D. Klimm and R. Fornari, "Theoretical Model for Calculation of Thermal Diffusion Factors in Diluted Binary Melts”, J. Chem. Phys. Letter, Vol. 444, pp. 202-204, 2007.

[139] I. Prigogine, L. de Brouckere and R, Amand, "Research on Thermodiffusion in Liquid Phase (First Submission)”, Physica Journal, Vol.16, pp. 577, 1950.

[140] S. Prager and H. Eyring, "Thermal Diffusion in Binary System”, J. Chem. Phys., Vol. 21, pp. 1347-1350, 1953.

[141] J. A. Madariaga, C. Santamaria, M. M. Bou-Ali, P. Urteaga and D. A. De Mezquia, "Measurement of Thermodiffusion Coefficient in n-Alkane Binary Mixtures: Composition Dependence”, J. Phys. Chem. B”, Vol. 114, pp. 6937-6942, 2010.

[142] T. E. Faber, An Introduction to the Theory of Liquid Metals, Cambridge University Press, London, 1972. 
[143] R. A. Howe and J. E. Enderby, “The Thermoelectric Power of Cerium, Lanthanum, Nickel and Cobalt in the Vicinity of the Melting Point”, J. Phys. F, Vol. 3, pp. L12-L14, 1973.

[144] N. Ashcroft and D. Mermin, Solid State Physics (Saunders College), Philadelphia, 1976.

[145] S. de Groot, Thermodynamics of Irreversible Processes, North-Holland, Amesterdam, 1951.

[146] J. Garai, “Physical Model for the Latent Heat”, Chem. Phys. Letter, Vol. 398, pp. 98, 2004.

[147] H. R. Thresh and A. F. Crawley, “The Viscosities of Lead, Tin, and Pb-Sn Alloys”, Metallurgical Transactions 1, pp.1531-1535, 1970.

[148] A. G. Ward, “The Viscosity of Pure Liquid”, Transactions of the Faraday Society, Vol. 33, pp. 88 - 97, 1937.

[149] J. M. Flinn, P. K. Gupta and T. A. Litovitz, "Mechanism of Volume Viscosity in the Liquid Metal System Lead-Bismuth”, J. Chem. Phys., Vol. 60, No. 11, pp. 4390-4395, 1974.

[150] S. Mudry, Yu. Plevachuk, V. Sklyarchuk and A. Yakymovych, "Viscosity of Bi-Zn Liquid Alloys”, J. Non-Cryst. Solids, Vol. 354, pp. 4415-4417, 2008.

[151] K. E. Spells, “The Determination of the Viscosity of Liquid Gallium Over an Extended Range of Temperature”, Proceedings of the Physical Society, Vol. 48, pp. 299-311, 1936.

[152] A. F. Guillermet, “Critical Evaluation of the Thermodynamic Properties of Cobalt”, Intel. J. Thermophys., Vol. 8, No. 4, pp. 481-510, 1987.

[153] H. J. Fisher and A. Phillips, “A Viscosity and Density of Liquid Lead-Tin and Antimony Cadmium Alloys”, J. Metals Trans. AIME, pp. 1060-1070, 1954. 
[154] D. R. Lide (Editor-in-Chief), CRC Handbook of Chemistry and Physics, $89^{\text {th }}$ Edition, 2008-2009.

[155] J. H. Lee and D. N. Lee, "Use of Thermodynamic Data to Calculate Surface Tension and Viscosity of Sn-Based Soldering Alloy SystemsJournal of Electronic Materials”, Vol. 30, No. 9, pp. 1112-1119, 2001.

[156] Y. P. Os’minin, “Density of Liquid Alkali Metals”, J. Eng. Phys. Thermophys., Vol. 8, No. 4, pp. 334-335, 1965.

[157] K. D. Sommer, and J. Poziemski, "Density, Thermal Expansion and Compressibility of Mercury”, Metrologia, Vol. 30, pp. 665-668, 1993-94.

[158] P. F Paradis, T. Ishikawa and N. Koike, "Density of Liquid Gold Measured by NonContact Technique”, Gold Bulletin, Vol. 3, pp. 242-245, 2008.

[159] L. J. T. M. Kempers, “A Comprehensive Thermodynamic Theory of the Soret Effect in a Multicomponent Gas, Liquid, or Solid”, J. Chem. Phys., Vol. 115, No. 14, pp. 6330, 2001.

[160] K. Shukla and A. Firoozabadi, “A New Model of Thermal Diffusion Coefficients in Binary Hydrocarbon Mixtures”, Industrial and Engineering Chemistry Research”, Vol. 37, pp. 3331, 1998.

[161] K.G. Honell and C.K. Hall, “A New Equation of State for Athermal Chains”, J. Chem. Phys., Vol. 90, pp. 1841, 1989.

[162] Y. Song, S.M. Lambert and J.M. Prausnitz, “Equation of State for Mixtures of Hard-Sphere Chains Including Copolymers”, Macromolecules, Vol. 27, pp. 441, 1994.

[163] N.F. Carnahan and K.E. Starling, "Equation of State for Nonattracting Rigid Spheres”, J. Chem. Phys., Vol. 51, pp. 635, 1969. 
[164] Y. Song and E.A. Mason, "Statistical-Mechanical Basis for Accurate Analytical Equations of State for Fluids”, Fluid Phase Equilibria, Vol. 75, pp. 105, 1992.

[165] E. Jafar-Salehi, and Z. Saghir, "Study of the Effect of Thermodiffusion during the Solidification process of a Molten Binary Metal”, Applied Mathematical Modelling, in Process.

[166] T. Boublik, "Equation of State of Hard Body Fluids”, J. Chem. Phys., Vol. 53, pp. 417, 1970.

[167] A.I. Abdulagatov, G.V. Stepanov and I.M. Abdulagatov, "The Critical Properties of Binary Mixtures Containing Carbon Dioxide: Krichevskii Parameter and Related Thermodynamic Properties”, High Temperature, Vol. 45, pp. 408, 2007.

[168] D. P. Tao, "Prediction of The Coordination Numbers of Liquid Metals", Thermochimica Acta, Vol. 363, pp. 105, 2000.

[169] H.W. Yang, D.P. Tao and Z.H. Zhou, "Prediction of the Mixing Enthalpies of Binary Liquid Alloys By Molecular Interaction Volume Model”, Acta Metallurgical Sinica. Vol. 21, No. 5, pp. 336-340, 2008.

[170] J.M. Prausnitz, R.N. Lichtenthaler and E.G. de Azevedo, Molecular Thermodynamics of Fluid-Phase Equilibria" $3^{\text {rd }}$ ed. Upper Saddle River Publisher, NJ, Prentice-Hall, 1999.

[171] E.E. Shpil'rain, V.I. Shkermontov, S.N. Skovorod'ko and A.G. Mozgovoi, “Activity of The Components of Binary Alloys of Alkali Metals: Na-K system”, High Temperature, Vol.40, No. 1, pp. 39-49, 2002.

[172] COMSOL Multiphysics Manual (4.3a), 2012.

[173] R. W. Lewis, P. Nithiarasu and K.N. Seetharamu, "Fundamentals of the Finite Element Method for Heat and Fluid Flow”, J. Wiley \&Sons Inc. New York, 2004. 
[174] T. Takemoto, A. Matsunawa and M. takahashi, "Tensile Test for estimation of Thermal Fatigue Properties of Solder Alloys”, J. Mater. Sci., Vol. 32, pp. 4077-4084, 1991.

[175] P.T. Vianco and F. J. Yost, Sandia Report, SAND 92-0211, Sandia Nat. Lab., pp. 1-9, April 1992.

[176] C. B. Lee, S. B. Jung, Y. E. Shin and C. C. Shur, "The Effect of Bi Concentration on Wettability of Cu Substrate by Sn-Bi Solder”, Mater. Transac., Vol. 42, pp. 751-755, 2001.

[177] J. Glazer, "Matallurgy of Low Temperature Pb-Free Solders for Electronic Assembly”, Intel. Mater. Rev., Vol. 40, pp. 65-93, 1995.

[178] F. Hua, Z. Mei and R. Glager, "Eutectic Sn-Bi as an Alternative to Pb-Free Solder", Proceeding of Electronics Components and Technology Conference, pp. 277-282, 1998.

[179] J. W. Yoon, C. B. Lee and S. B. Jung, "Interfacial Reactions Between Sn-58 mass\%Bi Eutectic Solder and (Cu, Electroless Ni-P/Cu) Substrate”, Mater. Transac., Vol. 43, No. 8, pp.1821-1826, 2002.

[180] Z. Mei and J. W. Morris, "Fatigue Lives on 60Sn/40Pb Solder Joints Made with Different Cooling Rates”, J. Electron. Mater., Vol.21, pp. 599-607, 1992.

[181] G. G. Graf, “Tin, Tin Alloys, and Tin Compounds”, in Ullmann's Encyclopedia of Industrial Chemistry, Wiley-VCH, Weinheim, 2000.

[182] B. A. Fowler and M. J. Sexton, "Bismuth”, In Nordberg, Gunnar. Handbook on the toxicology of metals. Academic Press. pp. 433, ISBN 978-0-12-369413-3, 2007.

[183] S. Hollinger and M. Lucke, "Influence of the Dufour effect on convection in binary gas mixtures”, Phys. Rev. E., Vol. 52, pp. 642-657, 1995. 
[184] S. Van Vaerenbergh, J. C. Legros and M. Hennenberg, "Reference Soret Coefficients of Natural Isotopes and Diluted Alloys of Tin”, J. Cryst. Growth, Vol. 158, pp. 369-376, 1996.

[185] P. C. Carman, "Fluid Flow Through a Granular Bed", Transaction of the Institution of Chemical Engineers, Vol. 15, pp.150, 1937.

[186] M. Hansen and K. Anderko, “Constitution of Binary Alloys”, 2nd edition, McGraw-Hill, New York, 1958.

[187] F. M. White, "Fluid Mechanics", 6 ${ }^{\text {th }}$ Edition, McGraw-Hill Series in Mechanical Engineering, pp. 287, 2008.

[188] L. H. Hodges and A. V. Astin, "Handbook of Mathematical Functions with Formulas, Graphs, and Mathematical Tables”, Applied Mathematic Series-55, 1964.

[189] M. Abramowitz and I. A. Stegun, "Handbook of Mathematical Functions with Formulas, Graphs, and Mathematical Tables”, 9th printing. New York, Dover, 1972.

[190] R. P. Kanwal, “Generalized Functions: Theory and Technique”, 2nd ed. Boston, MA: Birkhäuser, 1998.

[191] J. Spanier and K. B. Oldham, “The Unit-Step $u(x-a)$ and Related Functions”, Chapter 8; in An Atlas of Functions. Washington DC, Hemisphere, pp. 63-69, 1987.

[192] R. Bracewell, "Heaviside's Unit Step Function, $H(x)$, The Fourier Transform and Its Applications”, 3rd edition, McGraw-Hill, New York, pp. 61-65, 2000. 
